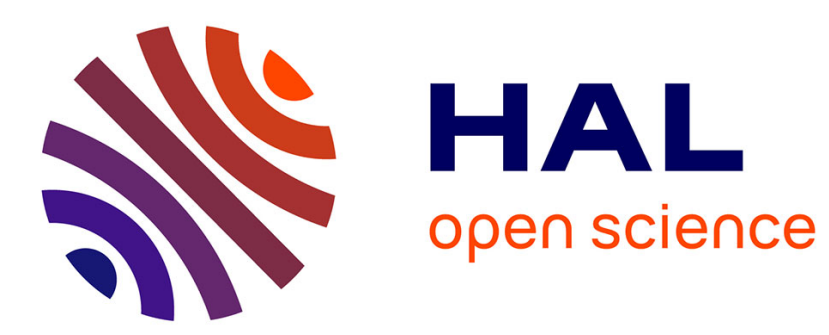

\title{
Convergence of approximations to stochastic scalar conservation laws
}

Sylvain Dotti, Julien Vovelle

\section{To cite this version:}

Sylvain Dotti, Julien Vovelle. Convergence of approximations to stochastic scalar conservation laws. Archive for Rational Mechanics and Analysis, 2018, 230 (2), pp.539-591. 10.1007/s00205-018-1252-2 . hal-01391069v5

\section{HAL Id: hal-01391069 https://hal.science/hal-01391069v5}

Submitted on 2 Jul 2018

HAL is a multi-disciplinary open access archive for the deposit and dissemination of scientific research documents, whether they are published or not. The documents may come from teaching and research institutions in France or abroad, or from public or private research centers.
L'archive ouverte pluridisciplinaire HAL, est destinée au dépôt et à la diffusion de documents scientifiques de niveau recherche, publiés ou non, émanant des établissements d'enseignement et de recherche français ou étrangers, des laboratoires publics ou privés. 


\title{
Convergence of Approximations to Stochastic Scalar Conservation Laws
}

\author{
Sylvain Dotti \& Julien VovelLe@
}

Communicated by A. BRESSAN

\begin{abstract}
We develop a general framework for the analysis of approximations to stochastic scalar conservation laws. Our aim is to prove, under minimal consistency properties and bounds, that such approximations are converging to the solution to a stochastic scalar conservation law. The weak probabilistic convergence mode is convergence in law, the most natural in this context. We use also a kinetic formulation and martingale methods. Our result is applied to the convergence of the finite volume method in the companion paper (Dotti and Vovelle in Convergence of the finite volume method for scalar conservation laws with multiplicative noise: an approach by kinetic formulation, 2016).
\end{abstract}

\section{Contents}

1. Introduction

2. Kinetic Solution

2.1. Definition

2.1.1 Predictable Sets and Functions

2.1.2 Random Measure, Solution

2.3. Left Limits of Generalized Solutions

3. Comparison, Uniqueness and Reduction of Generalized Solutions . . . . . . . .

3.1. Doubling of Variables . . . . . . . . . . . . . . . . . .

3.2. Uniqueness, Reduction of Generalized Solution . . . . . . . . . . . . .

4. Convergence of Approximate Solutions

4.1. Approximate Generalized Solutions

4.2. Martingale Characterization of the Stochastic Integral

4.3. Tightness

4.3.1 Compactness of the Young Measures

4.3.2 Compactness of the Random Measures

4.3.3 Tightness in the Skorokhod Space

JULIEN Vovelle was supported by ANR Projects STOSYMAP and STAB. 
4.4. Convergence of Approximate Generalized Solutions . . . . . . . . . . .

4.5. Proof of Theorem $4.6 \ldots \ldots \ldots \ldots \ldots$

4.5.1 State Space and Skorokhod's Theorem . . . . . . . . . . . . . .

4.5.2 Identification of the Limit: Càdlàg version . . . . . . . . . . . . . .

4.5.3 Identification of the Limit: Convergence of the Stochastic Integral . .

4.5.4 Identification of the Limit: Equation . . . . . . . . . . . . . . .

4.6. Pathwise Solutions and Almost Sure Convergence . . . . . . . . . . . .

5. Some Applications . . . . . . . . . . . . . . . . . . . .

5.1. Vanishing Viscosity Method

5.2. BGK Approximation

5.3. Approximation by the Finite Volume Method

References

\section{Introduction}

Let $\left(\Omega, \mathcal{F}, \mathbb{P},\left(\mathcal{F}_{t}\right),\left(\beta_{k}(t)\right)\right)$ be a stochastic basis and let $T>0$. Consider the first-order scalar conservation law with stochastic forcing

$$
\mathrm{d} u(x, t)+\operatorname{div}(A(u(x, t))) \mathrm{d} t=\Phi(x, u(x, t)) \mathrm{d} W(t), \quad x \in \mathbb{T}^{N}, t \in(0, T) .
$$

Equation (1.1) is periodic in the space variable: $x \in \mathbb{T}^{N}$ where $\mathbb{T}^{N}$ is the $N$ dimensional torus. The flux function $A$ in (1.1) is supposed to be of class $C^{2}$ : $A \in C^{2}\left(\mathbb{R} ; \mathbb{R}^{N}\right)$. We assume that $A$ and its derivatives have at most polynomial growth. The right-hand side of (1.1) is a stochastic increment in infinite dimension, and defined as follows (see [12] for the general theory): $W$ is a cylindrical Wiener process, $W=\sum_{k \geqq 1} \beta_{k} e_{k}$, where the coefficients $\beta_{k}$ are independent Brownian processes and $\left(e_{k}\right)_{k \geqq 1}$ is a complete orthonormal system in a Hilbert space $H$. For each $x \in \mathbb{T}^{N}, u \in \mathbb{R}, \Phi(x, u) \in L_{2}(H, \mathbb{R})$ is defined by $\Phi(x, u) e_{k}=g_{k}(x, u)$ where $g_{k}(\cdot, u)$ is a regular function on $\mathbb{T}^{N}$. Here, $L_{2}(H, K)$ denotes the set of Hilbert-Schmidt operators from the Hilbert space $H$ to another Hilbert space $K$. Since $K=\mathbb{R}$ in our case, this set is isomorphic to $H$, thus we may also define

$$
\Phi(x, u)=\sum_{k \geqq 1} g_{k}(x, u) e_{k},
$$

the action of $\Phi(x, u)$ on $e \in H$ being given by $\langle\Phi(x, u), e\rangle_{H}$. We assume $g_{k} \in$ $C\left(\mathbb{T}^{N} \times \mathbb{R}\right)$, with the bounds

$$
\begin{aligned}
\mathbf{G}^{2}(x, u) & =\|\Phi(x, u)\|_{H}^{2}=\sum_{k \geqq 1}\left|g_{k}(x, u)\right|^{2} \leqq D_{0}\left(1+|u|^{2}\right), \\
\|\Phi(x, u)-\Phi(y, v)\|_{H}^{2} & =\sum_{k \geqq 1}\left|g_{k}(x, u)-g_{k}(y, v)\right|^{2} \\
& \leqq D_{1}\left(|x-y|^{2}+|u-v| h(|u-v|)\right),
\end{aligned}
$$

where $x, y \in \mathbb{T}^{N}, u, v \in \mathbb{R}$, and $h$ is a continuous non-decreasing function on $\mathbb{R}_{+}$ such that $h(0)=0$. We assume also $0 \leqq h(z) \leqq 1$ for all $z \in \mathbb{R}_{+}$. 
Notation in what follows, we will use the convention of summation over repeated indices $k$. For example, we write $W=\beta_{k} e_{k}$ for the cylindrical Wiener process in (1.1).

This paper is a preliminary work to the analysis of convergence of the numerical approximation to (1.1) by the finite volume method with monotone fluxes, which is done in [16]. We give a general notion of a family of approximate solutions, see Definition 4.1, and explain what kind of convergence of such a family can be expected. Our main results in this regard are the theorem 4.6, about convergence to martingale solutions, and the theorem 4.15 , which gives criteria for convergence to pathwise solutions.

Problem (1.1) has already been studied in a series of papers. As in the deterministic case, the approach to the existence of solutions has been the vanishing viscosity method, see $[4,5,9,14,18,20,31,32,44]$ in particular. Approximation by the BGK method has been considered by HoFMANOVÁ [27]. Some results of convergence of numerical approximations to (1.1) (by the finite volume method in particular) have also been obtained in $[1-3,33,34,43]$.

The main difference between this present paper and all the works cited above is in the way to answer to the following question: when considering the convergence of approximations to (3.1), which mode of convergence regarding the sample variable $\omega$ is used? Here, we develop an approach based on convergence in law, while in the works referred to, weak convergence (in Lebesgue spaces, or in the sense of Young measures, $c f$. Section 2.2) is considered. ${ }^{1}$ Convergence in law is the natural mode of convergence for the random variables which manifest in the approximation to (1.1). Our approach based on convergence in law is successful because we work in the context of càdlàg processes. Another difference between this present paper and the references already quoted is that our formulation of a solution is weak in the space variable, but not weak in the time variable, see (2.6), (2.23) for example. This allows us to obtain convergence of approximations for each time $t$ (this is the last statement in Theorem 4.15) without making any regularity hypothesis on the initial datum at any moment. This paper is also a further development of the approach by kinetic formulation initiated in [14]. We need this in the companion paper [16] to obtain the convergence of the finite volume method with a standard CFL condition ( $c f$. our comment on the Kinetic formulation in the introduction section of [16]).

To complete this introduction, let us mention that the approximation of scalar conservation laws with stochastic flux (and not stochastic force) has also been considered in [21] (time-discrete scheme) and [39] (space discrete scheme). For the corresponding Cauchy problem, see [22,23,28,35-37].

The plan of the paper is as follows. Section 2 to Section 4 is devoted to the analysis of the Cauchy problem for (1.1); we introduce the kinetic formulation of the problem in Section 2, and prove a uniqueness result in Section 3. In Section 4, we develop a general approach to the analysis of convergence of approximate solutions to (1.1) based on martingale methods. In Section 5, we give some applications of our results of convergence of approximations.

${ }^{1}$ With the exception of [20], where quite a strong notion of solution is used however. 
Note that Sections 2 and 3 are in large part similar to Sections 2 and 3 in [14]. We must explain these similarities, and why we sometimes transfer, identically, some parts of [14], instead of simply making reference to it, and why, and in which places, we need to give new statements and new proofs as compared to [14]. This comparative analysis is given in Remark 3.3 at the end of Section 3.

\section{Kinetic Solution}

\subsection{Definition}

2.1.1. Predictable Sets and Functions For $T>0$, we denote by $\mathcal{B}([0, T])$ the Borel $\sigma$-algebra on $[0, T]$ and we denote by $\mathcal{P}_{T} \subset \mathcal{B}([0, T]) \otimes \mathcal{F}$ the predictable $\sigma$-algebra, [11, Section 2.2]. If $E$ is a Banach space, a process $(f(t))$ with values in $E$ is said to be weakly-predictable if the process $\left(\langle f(t), \varphi\rangle_{E, E^{\prime}}\right)$ is predictable for every $\varphi$ in the topological dual $E^{\prime}$. This is equivalent to saying that $f$ is weakly $\mathcal{P}_{T^{-}}$ measurable, in the sense of [45, Definition 1, p. 130]. Similarly, we can define the notion of strong predictability: the process $(f(t))$ is said to be strongly predictable if there exists a sequence of $E$-valued, $\mathcal{P}_{T}$-measurable simple functions which converges to $f$ at every point $(t, \omega)$ in a set of full measure in $[0, T] \times \Omega$. By Pettis' Theorem, [45, Theorem p. 131], the two notions of measurability coincide if $E$ is separable; in this case we say simply that they are "predictable".

Let us assume that $E$ is separable in order to introduce the following notations: let $p \in[1,+\infty)$. The set $L^{p}([0, T] \times \Omega ; E)$ is the set of $E$-valued, $\mathcal{B}([0, T]) \otimes \mathcal{F}$ measurable, Bochner integrable functions $f$ which satisfy

$$
\iint_{[0, T] \times \Omega}\|f(t, \omega)\|_{E}^{p} d(\mathcal{L} \times \mathbb{P})(t, \omega)<+\infty,
$$

where $\mathcal{L}$ is the Lebesgue measure on $[0, T]$. Equivalently, by definition of the product measure $\mathcal{L} \times \mathbb{P}$,

$$
\mathbb{E} \int_{0}^{T}\|f(t)\|_{E}^{p} \mathrm{~d} t<+\infty
$$

We denote by $L_{\mathcal{P}}^{p}([0, T] \times \Omega ; E)$ the set of functions $g$ in $L^{p}([0, T] \times \Omega ; E)$ which are equal $\mathcal{L} \times \mathbb{P}$-almost everywhere to a predictable function $f$. This is the case if, and only if, $\langle g, \varphi\rangle$ is equal $\mathcal{L} \times \mathbb{P}$-almost everywhere to $\langle f, \varphi\rangle$ for all $\varphi \in E^{\prime}$ (we use the following fact: $E$ being separable, there is a countable subset of $E^{\prime}$ that separates points in $E$, see [45, Lemma (1) p. 131]), so let us briefly consider the case $E=\mathbb{R}$. The class of processes in $L_{\mathcal{P}}^{p}([0, T] \times \Omega ; \mathbb{R})$ is analysed in [11, p. 66] or [42, p. 172]. In particular, if $X(t)$ is an adapted process with

$$
\mathbb{E} \int_{0}^{T}|X(t)|^{p} \mathrm{~d} t<+\infty
$$

then $X \in L_{\mathcal{P}}^{p}([0, T] \times \Omega ; \mathbb{R})$. A progressively measurable process $X$ in $L^{p}([0, T] \times$ $\Omega ; \mathbb{R})$ is also in $L_{\mathcal{P}}^{p}([0, T] \times \Omega ; \mathbb{R})$. 
Let $m \in \mathbb{N}^{*}$. In the case where $E$ is itself a Lebesgue space $E=L^{p}(D)$, where $D$ is an open subset of $\mathbb{R}^{m}$, we have $L^{p}\left([0, T] \times \Omega ; L^{p}(D)\right)=L^{p}(D \times[0, T] \times \Omega)$, where $D \times[0, T] \times \Omega$ is endowed with the product measure $\mathcal{L}_{m+1} \times \mathbb{P}\left(\mathcal{L}_{m}\right.$ being the $m$-dimensional Lebesgue measure), see [17, Section 1.8.1]. Similarly, we have

$$
L_{\mathcal{P}}^{p}\left([0, T] \times \Omega ; L^{p}(D)\right)=L_{\mathcal{P}}^{p}(D \times[0, T] \times \Omega),
$$

where $L_{\mathcal{P}}^{p}(D \times[0, T] \times \Omega)$ is the set of functions in $L^{p}(D \times[0, T] \times \Omega)$ which are equal $\mathcal{L}_{m} \times \mathcal{L} \times \mathbb{P}$-almost everywhere to a $\mathcal{B}(D) \times \mathcal{P}_{T}$-measurable function (here $\mathcal{B}(D)$ is the Borel $\sigma$-algebra on $D)$. We will apply these results with $D=(0,1)^{N}$, in which case, by periodic extension, we obtain

$$
L^{p}\left([0, T] \times \Omega ; L^{p}\left(\mathbb{T}^{N}\right)\right)=L^{p}\left(\mathbb{T}^{N} \times[0, T] \times \Omega\right),
$$

and it is similar for spaces $L_{\mathcal{P}}^{p}$.

2.1.2. Random Measure, Solution Let $\mathcal{M}_{b}\left(\mathbb{T}^{N} \times[0, T] \times \mathbb{R}\right)$ be the set of bounded Borel signed measures on $\mathbb{T}^{N} \times[0, T] \times \mathbb{R}$. We denote by $\mathcal{M}_{b}^{+}\left(\mathbb{T}^{N} \times\right.$ $[0, T] \times \mathbb{R})$ the subset of non-negative measures.

Definition 2.1. (Random measure). A map $m$ from $\Omega$ to $\mathcal{M}_{b}\left(\mathbb{T}^{N} \times[0, T] \times \mathbb{R}\right.$ ) is said to be a random signed measure (on $\mathbb{T}^{N} \times[0, T] \times \mathbb{R}$ ) if, for each $\phi \in$ $C_{b}\left(\mathbb{T}^{N} \times[0, T] \times \mathbb{R}\right),\langle m, \phi\rangle: \Omega \rightarrow \mathbb{R}$ is a random variable. If, almost surely, $m \in \mathcal{M}_{b}^{+}\left(\mathbb{T}^{N} \times[0, T] \times \mathbb{R}\right)$, we simply speak of random measure.

Let $m$ be a random measure with finite first-order moment

$$
\mathbb{E}\|m\|_{\mathrm{TV}}=\mathbb{E} m\left(\mathbb{T}^{N} \times[0, T] \times \mathbb{R}\right)<+\infty .
$$

Then $\mathbb{E} m$ is well defined and this is a bounded measure on $\mathbb{T}^{N} \times[0, T] \times \mathbb{R}$. In particular, it satisfies the following tightness condition:

$$
\lim _{R \rightarrow+\infty} \mathbb{E} m\left(\mathbb{T}^{N} \times[0, T] \times B_{R}^{c}\right)=0,
$$

where $B_{R}^{c}=\{\xi \in \mathbb{R},|\xi| \geqq R\}$. We note this fact here, since uniform versions of (2.3) will be required when considering sequences of random measures, see (4.15). We will also need the following result:

Lemma 2.1. (Atomic points). Let $m$ be a random measure with first moment (2.2). Let $\pi: \mathbb{T}^{N} \times[0, T] \times \mathbb{R} \rightarrow[0, T]$ denote the projection $(x, t, \xi) \mapsto t$. Let $\pi_{\#} m$ denote the push-forward of $m$ by $\pi$. Let $B_{\text {at }}$ denote the set of times $t$ such that the event " $t$ is an atom of $\pi_{\#} m$ " has positive probability:

$$
B_{\text {at }}=\left\{t \in[0, T] ; \mathbb{P}\left(\pi_{\#} m(\{t\})>0\right)>0\right\} .
$$

Then $B_{\text {at }}$ is at most countable.

Proof of Lemma 2.1. We also have

$$
B_{\text {at }}=\left\{t \in[0, T] ; \mathbb{E} \pi_{\#} m(\{t\})>0\right\} .
$$

The set $B_{\text {at }}$ is the set of atomic points of the measure $\mathbb{E} \pi_{\#} m$. It is therefore, at most, countable. 
The notion of a solution which we introduce below is based on the kinetic formulation of conservation laws introduced in [38]. In particular, for a given function $u$ of the variables $(x, t)$, we will need to consider the function

$$
\mathrm{f}(x, t, \xi):=\mathbf{1}_{u(x, t)>\xi,}
$$

which is the characteristic function of the subgraph of $u$. We often write $\mathrm{f}:=\mathbf{1}_{u>\xi}$ for short.

To be flexible enough, we have to impose a càdlàg property on solutions to (1.1) (see Item 2 in the following Definition 2.2). We will show however in Corollary 3.3 that solutions to (1.1) have continuous trajectories.

Definition 2.2. (Solution). Let $u_{0} \in L^{\infty}\left(\mathbb{T}^{N}\right)$. An $L^{1}\left(\mathbb{T}^{N}\right)$-valued stochastic process $(u(t))_{t \in[0, T]}$ is said to be a solution to (1.1) with initial datum $u_{0}$ if $u$ and $\mathrm{f}:=\mathbf{1}_{u>\xi}$ have the following properties:

1. $u \in L_{\mathcal{P}}^{1}\left(\mathbb{T}^{N} \times[0, T] \times \Omega\right)$,

2. for all $\varphi \in C_{c}^{1}\left(\mathbb{T}^{N} \times \mathbb{R}\right)$, almost surely, $t \mapsto\langle\mathrm{f}(t), \varphi\rangle$ is càdlàg,

3. for all $p \in[1,+\infty)$, there exists $C_{p} \geqq 0$ such that

$$
\mathbb{E}\left(\sup _{t \in[0, T]}\|u(t)\|_{L^{p}\left(\mathbb{T}^{N}\right)}^{p}\right) \leqq C_{p},
$$

4. there exists a random measure $m$ with first moment (2.2), such that for all $\varphi \in$ $C_{c}^{1}\left(\mathbb{T}^{N} \times \mathbb{R}\right)$, for all $t \in[0, T]$,

$$
\begin{aligned}
\langle\mathrm{f}(t), \varphi\rangle= & \left\langle\mathrm{f}_{0}, \varphi\right\rangle+\int_{0}^{t}\langle\mathrm{f}(s), a(\xi) \cdot \nabla \varphi\rangle \mathrm{d} s \\
& +\sum_{k \geqq 1} \int_{0}^{t} \int_{\mathbb{T}^{N}} g_{k}(x, u(x, s)) \varphi(x, u(x, s)) \mathrm{d} x \mathrm{~d} \beta_{k}(s) \\
& +\frac{1}{2} \int_{0}^{t} \int_{\mathbb{T}^{N}} \partial_{\xi} \varphi(x, u(x, s)) \mathbf{G}^{2}(x, u(x, s)) \mathrm{d} x \mathrm{~d} s-m\left(\partial_{\xi} \varphi\right)([0, t]),
\end{aligned}
$$

a.s., where $\mathrm{f}_{0}(x, \xi)=\mathbf{1}_{u_{0}(x)>\xi}, \mathbf{G}^{2}:=\sum_{k=1}^{\infty}\left|g_{k}\right|^{2}$ and $a(\xi):=A^{\prime}(\xi)$.

In (2.6), we have used the brackets $\langle\cdot, \cdot\rangle$ to denote the duality between $C_{c}^{\infty}\left(\mathbb{T}^{N} \times\right.$ $\mathbb{R})$ and the space of distributions over $\mathbb{T}^{N} \times \mathbb{R}$. In what follows, we will denote similarly the integral

$$
\langle F, G\rangle=\int_{\mathbb{T}^{N}} \int_{\mathbb{R}} F(x, \xi) G(x, \xi) \mathrm{d} x \mathrm{~d} \xi, \quad F \in L^{p}\left(\mathbb{T}^{N} \times \mathbb{R}\right), G \in L^{q}\left(\mathbb{T}^{N} \times \mathbb{R}\right),
$$

where $1 \leqq p \leqq+\infty$ and $q$ is the conjugate exponent of $p$. In (2.6) also, we have used (with $\phi=\partial_{\xi} \varphi$ ) the shorthand $m(\phi)$ to denote the Borel measure on $[0, T]$ defined by

$$
m(\phi): A \mapsto \int_{\mathbb{T}^{N} \times A \times \mathbb{R}} \phi(x, \xi) \mathrm{d} m(x, t, \xi), \quad \phi \in C_{b}\left(\mathbb{T}^{N} \times \mathbb{R}\right)
$$


for all $A$ Borel subset of $[0, T]$.

There is a last point to comment on in Definition 2.2, which is the measurability of the function $\sup _{t \in[0, T]}\|u(t)\|_{L^{p}\left(\mathbb{T}^{N}\right)}$ in (2.5). Let us denote by $\overline{\mathrm{f}}=1-\mathrm{f}=\mathbf{1}_{u \leqq \xi}$ the conjugate function of $\mathrm{f}$. By the identity

$$
|u|^{p}=\int_{\mathbb{R}}\left[\mathrm{f} \mathbf{1}_{\xi>0}+\overline{\mathrm{f}} \mathbf{1}_{\xi<0}\right] p|\xi|^{p-1} \mathrm{~d} \xi,
$$

we have, for $p \in[1,+\infty)$,

$$
\|u(t)\|_{L^{p}\left(\mathbb{T}^{N}\right)}^{p}=\sup _{\psi_{+} \in F_{+}, \psi_{-} \in F_{-}}\left\langle\mathrm{f}(t), \psi_{+}\right\rangle+\left\langle\overline{\mathrm{f}}(t), \psi_{-}\right\rangle,
$$

where the sup is taken over some countable sets $F_{+}$and $F_{-}$of functions $\psi$ chosen as follows: $F_{ \pm}=\left\{\psi_{n} ; n \geqq 1\right\}$, where $\left(\psi_{n}\right)$ is a sequence of non-negative functions in $C_{c}^{\infty}(\mathbb{R})$ which converges point-wise monotonically to $\xi \mapsto p\left|\xi^{ \pm}\right|^{p-1}$ if $p>1$ and to $\xi \mapsto \operatorname{sgn}_{ \pm}(\xi)$ if $p=1$. By (2.8), we have

$$
\sup _{t \in[0, T]}\|u(t)\|_{L^{p}\left(\mathbb{T}^{N}\right)}^{p}=\sup _{\psi \pm \in F_{ \pm}} \sup _{t \in[0, T]}\left\langle\mathrm{f}(t), \psi_{+}\right\rangle+\left\langle\overline{\mathrm{f}}(t), \psi_{-}\right\rangle .
$$

By Item 2 in Definition 2.2, we know that the function

$$
\sup _{t \in[0, T]}\left\langle\mathrm{f}(t), \psi_{+}\right\rangle+\left\langle\overline{\mathrm{f}}(t), \psi_{-}\right\rangle
$$

is $\mathcal{F}$-measurable for all $\psi_{ \pm} \in F_{ \pm}$. Indeed, the sup over [0,T] of a càdlàg function is the sup of the function on any dense countable subset of $[0, T]$ containing the terminal point $T$. By (2.9), the function $\sup _{t \in[0, T]}\|u(t)\|_{L^{p}\left(\mathbb{T}^{N}\right)}$ is measurable.

Remark 2.1. (Initial condition). A limiting argument based on (2.6) leads to the following initial condition for $\mathrm{f}(t)$ :

$$
\mathrm{f}(0)=\mathrm{f}_{0}+\partial_{\xi} m_{0}, \quad \text { a.s. },
$$

where $m_{0}$ is the restriction of $m$ to $\mathbb{T}^{N} \times\{0\} \times \mathbb{R}$. It is thus not obvious that (2.6) entails the expected initial condition $f(0)=f_{0}$. This is the case however (and, therefore, $m_{0} \equiv 0$ a.s.), due to Proposition 2.11 and Corollary 2.12; see also the discussion on the same topic in Section 5 of [10].

Proposition 2.2. (Mass of the random measure). Let $u_{0} \in L^{\infty}\left(\mathbb{T}^{N}\right)$. Let $(u(t))_{t \in[0, T]}$ be a solution to (1.1) with initial datum $u_{0}$. Then the total mass of the measure $m$ in (2.6) is

$$
\begin{aligned}
m\left(\mathbb{T}^{N} \times[0, T] \times \mathbb{R}=\right. & \frac{1}{2}\left\|u_{0}\right\|_{L^{2}\left(\mathbb{T}^{N}\right)}^{2}-\frac{1}{2}\|u(T)\|_{L^{2}\left(\mathbb{T}^{N}\right)}^{2} \\
& +\sum_{k \geqq 1} \int_{0}^{T} \int_{\mathbb{T}^{N}} g_{k}(x, u(x, t)) u(x, t) \mathrm{d} x \mathrm{~d} \beta_{k}(t) \\
& +\frac{1}{2} \int_{0}^{T} \int_{\mathbb{T}^{N}} \mathbf{G}^{2}(x, u(x, t)) \mathrm{d} x \mathrm{~d} t,
\end{aligned}
$$

almost surely. 
Proof of Proposition 2.2. We start from (2.6), which we apply with a test-function $\varphi$ independent on $x$. By substracting $\left\langle\mathbf{1}_{0>\xi}, \varphi\right\rangle$ from both sides of the equation, we obtain

$$
\begin{aligned}
\langle\chi(t), \varphi\rangle= & \left\langle\chi_{0}, \varphi\right\rangle+\sum_{k \geqq 1} \int_{0}^{t} \int_{\mathbb{T}^{N}} g_{k}(x, u(x, s)) \varphi(x, u(x, s)) \mathrm{d} x \mathrm{~d} \beta_{k}(s) \\
& +\frac{1}{2} \int_{0}^{t} \int_{\mathbb{T}^{N}} \partial \xi \varphi(x, u(x, s)) \mathbf{G}^{2}(x, u(x, s)) \mathrm{d} x \mathrm{~d} s-m\left(\partial_{\xi} \varphi\right)([0, t]),
\end{aligned}
$$

where $\chi(x, t, \xi)=\mathrm{f}(x, t, \xi)-\mathbf{1}_{0>\xi}, \chi_{0}(x, \xi)=\mathrm{f}_{0}(x, \xi)-\mathbf{1}_{0>\xi}$ are the traditional kinetic functions used in [41] for example. We use then an approximation argument to apply (2.11) with $\varphi(x, \xi)=\xi$. This gives (2.10).

\subsection{Generalized Solutions}

With the purpose of preparing the proof of existence of a solution, we introduce the following definitions:

Definition 2.3. (Young measure). Let $(X, \mathcal{A}, \lambda)$ be a finite measure space. Let $\mathcal{P}_{1}(\mathbb{R})$ denote the set of probability measures on $\mathbb{R}$. We say that a map $v: X \rightarrow$ $\mathcal{P}_{1}(\mathbb{R})$ is a Young measure on $X$ if, for all $\phi \in C_{b}(\mathbb{R})$, the map $z \mapsto\left\langle v_{z}, \phi\right\rangle$ from $X$ to $\mathbb{R}$ is measurable. We say that a Young measure $v$ vanishes at infinity if, for every $p \geqq 1$,

$$
\int_{X} \int_{\mathbb{R}}|\xi|^{p} \mathrm{~d} \nu_{z}(\xi) \mathrm{d} \lambda(z)<+\infty .
$$

Proposition 2.3. (An alternative definition of Young measures). Let $(X, \mathcal{A}, \lambda)$ be a measure space with $\lambda(X)=1$. Let $\mathcal{L}$ be the Lebesgue measure on $\mathbb{R}$ and let $\mathcal{Y}^{1}$ be the set of probability measures $v$ on $(X \times \mathbb{R}, \mathcal{A} \times \mathcal{B}(\mathbb{R}))$ such that $\pi_{\#} v=\lambda$, where $\pi_{\#} v$ is the push forward of $v$ by the projection $\pi: X \times \mathbb{R} \rightarrow X$. Then $\mathcal{Y}^{1}$ is the set of Young measures as defined in Definition 2.3.

For the proof of this result, which uses the disintegration theorem, we refer to the discussion in [8, p. 19-20] on the spaces $\mathcal{Y}^{1}$ and $\mathcal{Y}_{\text {dis }}^{1}$ ("dis" for "disintegration": this corresponds to the Definition 2.3). Note that there is no loss of generality in assuming $\lambda(X)=1$.

Definition 2.4. (Kinetic function). Let $(X, \mathcal{A}, \lambda)$ be a finite measure space. A measurable function $f: X \times \mathbb{R} \rightarrow[0,1]$ is said to be a kinetic function if there exists a Young measure $v$ on $X$ that vanishes at infinity such that, for $\lambda$-almost everywhere $z \in X$, for all $\xi \in \mathbb{R}$,

$$
f(z, \xi)=v_{z}(\xi,+\infty)
$$

We say that $f$ is an equilibrium if there exists a measurable function $u: X \rightarrow \mathbb{R}$ such that $f(z, \xi)=\mathrm{f}(z, \xi)=\mathbf{1}_{u(z)>\xi}$ almost everywhere, or, equivalently, $\nu_{z}=\delta_{\xi=u(z)}$ for almost every $z \in X$. 
Definition 2.5. (Conjugate function). If $f: X \times \mathbb{R} \rightarrow[0,1]$ is a kinetic function, we denote by $\bar{f}$ the conjugate function $\bar{f}:=1-f$.

We also denote by $\chi_{f}$ the function defined by $\chi_{f}(z, \xi)=f(z, \xi)-\mathbf{1}_{0>\xi}$. This correction to $f$ is integrable on $\mathbb{R}$. Actually, it is decreasing faster than any power of $|\xi|$ at infinity. Indeed, we have $\chi_{f}(z, \xi)=-v_{z}(-\infty, \xi)$ when $\xi<0$ and $\chi_{f}(z, \xi)=v_{z}(\xi,+\infty)$ when $\xi>0$. Therefore

$$
|\xi|^{p} \int_{X}\left|\chi_{f}(z, \xi)\right| \mathrm{d} \lambda(z) \leq \int_{X} \int_{\mathbb{R}}|\zeta|^{p} \mathrm{~d} \nu_{z}(\zeta) \mathrm{d} \lambda(z)<\infty
$$

for all $\xi \in \mathbb{R}, 1 \leqq p<+\infty$.

The so-called kinetic functions appear naturally when one examines the stability of a sequence of solutions to (1.1). We discuss this topic in details in Section 4, but let us already mention the following compactness result:

Theorem 2.4. (Compactness of Young measures). Let $(X, \mathcal{A}, \lambda)$ be a finite measure space such that $\mathcal{A}$ is countably generated. Let $\left(v^{n}\right)$ be a sequence of Young measures on $X$ satisfying (2.12) uniformly for some $p \geq 1$ :

$$
\sup _{n} \int_{X} \int_{\mathbb{R}}|\xi|^{p} \mathrm{~d} \nu_{z}^{n}(\xi) \mathrm{d} \lambda(z)<+\infty .
$$

Then there exists a Young measure $v$ on $X$ and a subsequence still denoted $\left(v^{n}\right)$ such that, for all $h \in L^{1}(X)$, for all $\phi \in C_{b}(\mathbb{R})$,

$$
\lim _{n \rightarrow+\infty} \int_{X} h(z) \int_{\mathbb{R}} \phi(\xi) \mathrm{d} \nu_{z}^{n}(\xi) \mathrm{d} \lambda(z)=\int_{X} h(z) \int_{\mathbb{R}} \phi(\xi) \mathrm{d} \nu_{z}(\xi) \mathrm{d} \lambda(z) .
$$

The convergence (2.15) is the convergence for the $\tau_{\mathcal{Y}^{1}}^{W}$ topology defined in [8, p. 21]. By [8, Corollary 4.3.7], (2.14) implies that the set $\left\{v_{n} ; n \in \mathbb{N}\right\}$ is $\tau_{\mathcal{Y}^{1}}^{W}$ relatively compact, and for this result, it is not necessary to assume that $\mathcal{A}$ is countably generated. This latter hypothesis is used as a criteria of metrizability of $\tau_{\mathcal{Y}^{1}}^{W}$, [8, Proposition 2.3.1]. A consequence of Theorem 2.4 is the following proposition:

Corollary 2.5. (Compactness of kinetic functions). Let $(X, \mathcal{A}, \lambda)$ be a finite measure space such that $\mathcal{A}$ is countably generated. Let $\left(f_{n}\right)$ be a sequence of kinetic functions on $X \times \mathbb{R}: f_{n}(z, \xi)=v_{z}^{n}(\xi,+\infty)$ where $v^{n}$ are Young measures on $X$ satisfying (2.14). Then there exists a kinetic function $f$ on $X \times \mathbb{R}$ (related to the Young measure $v$ in Theorem 2.4 by the formula $\left.f(z, \xi)=v_{z}(\xi,+\infty)\right)$ such that, up to a subsequence, $f_{n} \rightarrow f$ in $L^{\infty}(X \times \mathbb{R})$ weak-*.

We will also need

Lemma 2.6. (Convergence to an equilibrium). Let $(X, \mathcal{A}, \lambda)$ be a finite measure space. Let $p>1$. Let $\left(f_{n}\right)$ be a sequence of kinetic functions on $X \times \mathbb{R}: f_{n}(z, \xi)=$ $v_{z}^{n}(\xi,+\infty)$ where $v^{n}$ are Young measures on $X$ satisfying (2.14). Let $f$ be a kinetic 
function on $X \times \mathbb{R}$ such that $f_{n} \rightarrow f$ in $L^{\infty}(X \times \mathbb{R})$ weak-*. Assuming that $f$ is an equilibrium, $f(z, \xi)=\mathrm{f}(z, \xi)=\mathbf{1}_{u(z)>\xi}$, and letting

$$
u_{n}(z)=\int_{\mathbb{R}} \xi \mathrm{d} \nu_{z}^{n}(\xi)
$$

then, for all $1 \leqq q<p, u_{n} \rightarrow u$ in $L^{q}(X)$ strong.

Proof of Corollary 2.5. We apply the theorem 2.4. The convergence of $\left(v^{n}\right)$, which means that

$$
\left(z \mapsto\left\langle v_{z}^{n}, \phi\right\rangle\right) \rightarrow\left(z \mapsto\left\langle v_{z}, \phi\right\rangle\right) \text { in } L^{\infty}(X) \text { weak }-*
$$

for all $\phi \in C_{b}(\mathbb{R})$, has the consequence that

$$
\int_{X} \int_{\mathbb{R}} \alpha(z, \xi) \mathrm{d} \nu_{z}^{n}(\xi) \mathrm{d} \lambda(z) \rightarrow \int_{X} \int_{\mathbb{R}} \alpha(z, \xi) \mathrm{d} v_{z}(\xi) \mathrm{d} \lambda(z)
$$

for every bounded Carathéodory integrand $\alpha$. This is a consequence of the identity $\tau_{\mathcal{Y}^{1}}^{M}=\tau_{\mathcal{Y}^{1}}^{W}$ in the portmanteau theorem [8, Theorem 2.1.3] (see also [8, Lemma 1.2.3] about Carathéodory integrands). We apply (2.17) to

$$
\alpha(z, \xi)=\int_{-\infty}^{\xi} \varphi(z, \zeta) \mathrm{d} \zeta
$$

where $\varphi \in L^{1} \cap L^{\infty}(X \times \mathbb{R})$, and apply also the Fubini theorem to obtain

$$
\iint_{X \times \mathbb{R}} f_{n}(z, \xi) \varphi(z, \xi) \mathrm{d} \lambda(z) \mathrm{d} \xi \rightarrow \iint_{X \times \mathbb{R}} f(z, \xi) \varphi(z, \xi) \mathrm{d} \lambda(z) \mathrm{d} \xi .
$$

Using the bound by 1 on the $L^{\infty}$ norm of $f_{n}$ and $f$, we deduce by an argument of density that (2.18) holds true when $\varphi \in L^{1}(X \times \mathbb{R})$.

Proof of Lemma 2.6. Let $r \in[1,+\infty]$. By choosing $\theta=\phi$ and $\gamma$ as a test function in $z$ in (2.16), and by use of a standard approximation procedure, we have

$$
\int_{X} \int_{\mathbb{R}} \theta(\xi) \mathrm{d} \nu_{z}^{n}(\xi) \gamma(z) \mathrm{d} \lambda(z) \rightarrow \int_{X} \theta(u(z)) \gamma(z) \mathrm{d} \lambda(z)
$$

for all $\theta \in C(\mathbb{R})$ and $\gamma \in L^{r}(X)$ such that

$$
\sup _{n}\left\|\int_{\mathbb{R}} \theta(\xi) \mathrm{d} v_{z}^{n}(\xi)\right\|_{L^{r^{\prime}(X)}}<+\infty
$$

where $r^{\prime}$ is the conjugate exponent to $r$. Let us assume first that $p>2$ and let us prove the convergence of $\left(u_{n}\right)$ to $u$ in $L^{2}(X)$. By (2.19), taking $r=2, \theta(\xi)=\xi$ and $\gamma \in L^{2}(X)$, we obtain the weak convergence of $\left(u_{n}\right)$ to $u$ in $L^{2}(X)$. By developing the scalar product

$$
\left\|u-u_{n}\right\|_{L^{2}(X)}^{2}=\|u\|_{L^{2}(X)}^{2}+\left\|u_{n}\right\|_{L^{2}(X)}^{2}-2\left\langle u, u_{n}\right\rangle_{L^{2}(X)},
$$


we see that it is sufficient, in order to establish the strong convergence, to prove that

$$
\limsup _{n \rightarrow+\infty}\left\|u_{n}\right\|_{L^{2}(X)}^{2} \leqq\|u\|_{L^{2}(X)}^{2}
$$

We obtain (2.20) by the Jensen inequality, which gives

$$
\left\|u_{n}\right\|_{L^{2}(X)}^{2}=\int_{X}\left|\int_{\mathbb{R}} \xi \mathrm{d} \nu_{z}^{n}(\xi)\right|^{2} \mathrm{~d} \lambda(z) \leqq \int_{X} \int_{\mathbb{R}}|\xi|^{2} \mathrm{~d} \nu_{x}^{n}(\xi) \mathrm{d} \lambda(z) .
$$

Indeed the right-hand side of (2.21) is converging to $\|u\|_{L^{2}(X)}^{2}$ (here, we apply (2.19) with $\theta(\xi)=\xi^{2}$ and $\left.\gamma(z)=1\right)$. Still assuming $p>2$, the remaining cases $1 \leqq q<p$ are deduced from the result for $p=2$ by interpolation and by the uniform bound on $\left\|u_{n}\right\|_{L^{p}(X)}$. Let us consider the case $p \leqq 2$ now. Let us introduce the truncate functions and truncate sequence $\left(u_{n}^{R}\right)$ as follows:

$$
T_{R}(\xi):=\min (R, \max (-R, \xi)), \quad u_{n}^{R}(z)=\int_{\mathbb{R}} T_{R}(\xi) \mathrm{d} \nu_{z}^{n}(\xi) .
$$

One checks that the study done for $p>2$ can be applied to established the convergence $u_{n}^{R} \rightarrow T_{R}(u)$ in $L^{r}(X)$ strong for every $r<+\infty$. Then we use the estimate

$$
\left|u_{n}^{R}(z)-u_{n}(z)\right| \leqq \int_{|\xi|>R}|R-\xi| \mathrm{d} \nu_{z}^{n}(\xi) \leqq 2 \int_{|\xi|>R}|\xi| \mathrm{d} \nu_{z}^{n}(\xi),
$$

from which follows, for $1 \leqq q<p$, by the Jensen inequality

$$
\left\|u_{n}^{R}-u_{n}\right\|_{L^{q}(X)}^{q} \leqq 2^{q} \int_{X} \int_{|\xi|>R}|\xi|^{q} d \nu_{z}^{n}(\xi) \mathrm{d} \lambda(z) \leqq \frac{2^{q}}{R^{p-q}} \int_{X} \int_{\mathbb{R}}|\xi|^{p} d \nu_{z}^{n} \mathrm{~d} \lambda(z),
$$

and, thanks to (2.14), the uniform bound

$$
\left\|u_{n}^{R}-u_{n}\right\|_{L^{q}(X)} \leqq \frac{2}{R^{p / q-1}} \sup _{n} \int_{X} \int_{\mathbb{R}}|\xi|^{p} d v_{z}^{n} \mathrm{~d} \lambda(z) .
$$

We have also $T_{R}(u) \rightarrow u$ in $L^{q}(X)$ when $R \rightarrow+\infty$. Gathering the different results of convergence, we obtain $u_{n} \rightarrow u$ in $L^{q}(X)$.

In the deterministic setting, if $\left(u_{n}(t)\right)$ is a sequence of solutions to (1.1), then, due to natural bounds and to Theorem 2.4, the sequence of Young measures $\delta_{u_{n}(x, t)}$ on $X:=\mathbb{T}^{N}$ (consider that $t$ is fixed here) has, up to a subsequence, a limit $v_{t}$. Then every non-linear expression $\phi\left(u_{n}(t)\right)$ for $\phi \in C_{b}(\mathbb{R})$ will converge to $\left\langle v_{t}, \phi\right\rangle$ in the sense of (2.15). This is why it is natural (cf. [15]), for such non-linear problems as (1.1), to introduce the following generalization to Definition 2.2:

Definition 2.6. (Generalized solution). Let $f_{0}: \mathbb{T}^{N} \times \mathbb{R} \rightarrow[0,1]$ be a kinetic function. An $L^{\infty}\left(\mathbb{T}^{N} \times \mathbb{R} ;[0,1]\right)$-valued process $(f(t))_{t \in[0, T]}$ is said to be a generalized solution to (1.1) with initial datum $f_{0}$ if $f(t)$ and $v_{t}:=-\partial_{\xi} f(t)$ have the following properties: 
1. for all $t \in[0, T]$, almost surely, $f(t)$ is a kinetic function, and, for all $R>0$, $f \in L_{\mathcal{P}}^{1}\left(\mathbb{T}^{N} \times(0, T) \times(-R, R) \times \Omega\right)$,

2. for all $\varphi \in C_{c}^{1}\left(\mathbb{T}^{N} \times \mathbb{R}\right)$, almost surely, the map $t \mapsto\langle f(t), \varphi\rangle$ is càdlàg,

3. for all $p \in[1,+\infty)$, there exists $C_{p} \geqq 0$ such that

$$
\mathbb{E}\left(\sup _{t \in[0, T]} \int_{\mathbb{T}^{N}} \int_{\mathbb{R}}|\xi|^{p} \mathrm{~d} v_{x, t}(\xi) \mathrm{d} x\right) \leqq C_{p},
$$

4. there exists a random measure $m$ with first moment (2.2), such that for all $\varphi \in$ $C_{c}^{1}\left(\mathbb{T}^{N} \times \mathbb{R}\right)$, for all $t \in[0, T]$, almost surely,

$$
\begin{aligned}
\langle f(t), \varphi\rangle= & \left\langle f_{0}, \varphi\right\rangle+\int_{0}^{t}\left\langle f(s), a(\xi) \cdot \nabla_{x} \varphi\right\rangle \mathrm{d} s \\
& +\int_{0}^{t} \int_{\mathbb{T}^{N}} \int_{\mathbb{R}} g_{k}(x, \xi) \varphi(x, \xi) \mathrm{d} v_{x, s}(\xi) \mathrm{d} x \mathrm{~d} \beta_{k}(s) \\
& +\frac{1}{2} \int_{0}^{t} \int_{\mathbb{T}^{N}} \int_{\mathbb{R}} \mathbf{G}^{2}(x, \xi) \partial_{\xi} \varphi(x, \xi) \mathrm{d} v_{x, s}(\xi) \mathrm{d} x \mathrm{~d} s-m\left(\partial_{\xi} \varphi\right)([0, t]) .
\end{aligned}
$$

Let us make a comment about notations: for each $t \in[0, T]$, we have a Young measure $v_{t}$ on $\mathbb{T}^{N}$. This gives us a set of probability measures $\left(v_{x, t}\right)_{x \in \mathbb{T}^{N}}$, as they appear in the second line of (2.23). There is something misleading in the use of the notation $v_{x, t}$, which conveys the idea that we are considering a Young measure $v$ with index space $\mathbb{T}^{N} \times(0, T)$. Such a modification of the point of view is admissible however, and we will use it fully in Section 4.3.1 to obtain the convergence of sequences of Young measures. Indeed, due to item 1 and to the fact that, for all $t \in(0, T)$, for almost every $x \in \mathbb{T}^{N}$, a.s.,

$$
\int_{\mathbb{R}} \phi(\xi) \mathrm{d} v_{x, t}(\xi)=\int_{\mathbb{R}} f(x, t, \xi) \phi^{\prime}(\xi) \mathrm{d} \xi,
$$

if $\phi \in C_{c}^{1}(\mathbb{R})$, the map $(\omega, x, t) \mapsto\left\langle v_{x, t}, \phi\right\rangle$ is measurable (and in $L_{\mathcal{P}}^{1}\left(\mathbb{T}^{N} \times\right.$ $(0, T) \times \Omega)$ actually). By the Fubini theorem, we deduce that, almost surely, $(x, t) \mapsto\left\langle v_{x, t}, \phi\right\rangle$ is measurable when $\phi \in C_{c}^{1}(\mathbb{R})$. By an argument of density, this holds true when $\phi \in C_{b}(\mathbb{R})$.

This point about the status of $v_{x, t}$ being clear, we have now also to justify that the stochastic integral in (2.23) is well-defined. The bound (2.22) implies

$$
\mathbb{E}\left(\int_{0}^{T} \int_{\mathbb{T}^{N}} \int_{\mathbb{R}}|\xi|^{p} \mathrm{~d} v_{x, t}(\xi) \mathrm{d} x\right) \leqq C_{p} T
$$


Using successively, Jensen's Inequality, the growth hypothesis (1.3), and (2.24) with $p=2$, we obtain, for $\varphi \in C_{c}^{1}\left(\mathbb{T}^{N} \times \mathbb{R}\right)$,

$$
\begin{aligned}
& \mathbb{E} \int_{0}^{T} \sum_{k \geqq 1}\left|\int_{\mathbb{T}^{N}} \int_{\mathbb{R}} g_{k}(x, \xi) \varphi(x, \xi) \mathrm{d} v_{x, t}(\xi) \mathrm{d} x\right|^{2} \\
& \leq \mathbb{E} \int_{0}^{T} \sum_{k \geqq 1} \int_{\mathbb{T}^{N}} \int_{\mathbb{R}}\left|g_{k}(x, \xi) \varphi(x, \xi)\right|^{2} \mathrm{~d} v_{x, t}(\xi) \mathrm{d} x \\
& \quad=\mathbb{E} \int_{0}^{T} \int_{\mathbb{T}^{N}} \int_{\mathbb{R}} \mathbf{G}^{2}(x, \xi)|\varphi(x, \xi)|^{2} \mathrm{~d} v_{x, t}(\xi) \mathrm{d} x \\
& \leq\|\varphi\|_{L_{x, \xi}^{\infty} D_{0}}^{2}\left(1+C_{2} T\right) .
\end{aligned}
$$

The fact that

$$
t \mapsto \int_{\mathbb{T}^{N}} \int_{\mathbb{R}} g_{k}(x, \xi) \varphi(x, \xi) \mathrm{d} v_{x, t}(\xi) \mathrm{d} x
$$

is predictable is a consequence of item 1 . To sum up, we have proved the following result:

Lemma 2.7. (Admissible integrand). Let $f_{0}: \mathbb{T}^{N} \times \mathbb{R} \rightarrow[0,1]$ be a kinetic function. Let $(f(t))_{t \in[0, T]}$ be a generalized solution to (1.1) with initial datum $f_{0}$. Then, for all $\varphi \in C_{c}^{1}\left(\mathbb{T}^{N} \times \mathbb{R}\right)$ the $l^{2}\left(\mathbb{N}^{*}\right)$-valued process

$$
t \mapsto\left(\int_{\mathbb{T}^{N}} \int_{\mathbb{R}} g_{k}(x, \xi) \varphi(x, \xi) \mathrm{d} \nu_{x, t}(\xi) \mathrm{d} x\right)_{k \geqq 1}
$$

is in $L_{\mathcal{P}}^{2}\left([0, T] \times \Omega ; l^{2}\left(\mathbb{N}^{*}\right)\right)$.

Let us now state a simple result of reduction from a generalized solution to a mere solution.

Proposition 2.8. Let $u_{0} \in L^{\infty}\left(\mathbb{T}^{N}\right)$. Let $(f(t))_{t \in[0, T]}$ be a generalized solution to (1.1) with initial datum $\mathbf{1}_{u_{0}>\xi}$. If for all $t \in[0, T], f(t)$ is an equilibrium, then

$$
f(x, t, \xi, \omega)=\mathrm{f}(x, t, \xi, \omega)=\mathbf{1}_{u(x, t, \omega)>\xi}
$$

for almost every $(x, \xi, \omega) \in \mathbb{T}^{N} \times \mathbb{R} \times \Omega$, then $(u(t))_{t \in[0, T]}$ is a solution to $(1.1)$ with initial datum $u_{0}$.

Proof of Proposition 2.8. Under (2.26), we have $v_{t}=\delta_{u(t)}$ a.s. From (2.24) with $p=1$, we deduce that $u \in L^{1}\left(\mathbb{T}^{N} \times(0, T) \times \Omega\right)$. Since

$$
u(x, t)=\int_{\mathbb{R}} \xi \mathrm{d} v_{x, t}(\xi),
$$

we obtain $u \in L_{\mathcal{P}}^{1}\left(\mathbb{T}^{N} \times(0, T) \times \Omega\right)$ as a consequence of Item 1 in Definition 2.6. We also have

$$
\langle\mathrm{f}(t), \varphi\rangle=\int_{\mathbb{T}^{N}} \int_{\mathbb{R}} \psi(x, \xi) \mathrm{d} v_{x, t}(\xi) \mathrm{d} x, \quad \psi(x, \xi):=\int_{-\infty}^{\xi} \varphi(x, \zeta) \mathrm{d} \zeta
$$


for all $\varphi \in C_{c}^{\infty}\left(\mathbb{T}^{N} \times \mathbb{R}\right)$. Therefore Item 2 in Definition 2.2 follows from Item 2 in Definition 2.6. Using the identity

$$
\int_{\mathbb{T}^{N}}|u(t, x)|^{p} \mathrm{~d} x=\int_{\mathbb{T}^{N}} \int_{\mathbb{R}}|\xi|^{p} \mathrm{~d} \nu_{x, t}(\xi) \mathrm{d} x
$$

we obtain Item 3 in Definition 2.2. Item 4 in Definition 2.2 follows from Item 4 in Definition 2.6.

We will show in Theorem 3.2 that (2.26), which we give as an hypothesis in Proposition 2.8 , is automatically satisfied by any generalized solution starting from an equilibrium $f_{0}=\mathrm{f}_{0}=\mathbf{1}_{u_{0}>\xi}$.

We conclude this section with the following result, used in the proof of Corollary 3.3:

Lemma 2.9. (Distance to equilibrium). Let $(X, \lambda)$ be a finite measure space. Let $f: X \times \mathbb{R} \rightarrow[0,1]$ be a kinetic function. Then

$$
m(\xi):=\int_{-\infty}^{\xi}\left(\mathbf{1}_{u>\zeta}-f(\zeta)\right) \mathrm{d} \zeta, \text { where } u:=\int_{\mathbb{R}} \chi_{f}(\zeta) \mathrm{d} \zeta
$$

is well defined and non-negative.

Note in particular that the difference $f(\xi)-\mathbf{1}_{u>\xi}$ writes $\partial_{\xi} m$ where $m \geqq 0$.

Proof of Lemma 2.9. Let $v_{z}=-\partial_{\xi} f(z, \cdot), z \in X$. By Jensen's Inequality, we have

$$
H\left(\int_{\mathbb{R}} \zeta \mathrm{d} \nu_{z}(\zeta)\right) \leqq \int_{\mathbb{R}} H(\zeta) \mathrm{d} \nu_{z}(\zeta)
$$

for all convex sub-linear function $H: \mathbb{R} \rightarrow \mathbb{R}$. Note that

$$
u(z)=\int_{\mathbb{R}} f(z, \zeta)-\mathbf{1}_{0>\zeta} \mathrm{d} \zeta=\int_{\mathbb{R}} \zeta \mathrm{d} v_{z}(\zeta)
$$

by integration by parts. By integration by parts, we also have, for all sub-linear function $H \in C^{1}(\mathbb{R})$,

$$
\int_{\mathbb{R}} H(\zeta) \mathrm{d} v_{z}(\zeta)=H(0)+\int_{\mathbb{R}} H^{\prime}(\zeta)\left(f(z, \zeta)-\mathbf{1}_{0>\zeta}\right) \mathrm{d} \zeta
$$

and

$$
H(u(z))=\int_{\mathbb{R}} H(\zeta) \mathrm{d} \delta_{u(z)}(\zeta)=H(0)+\int_{\mathbb{R}} H^{\prime}(\zeta)\left(\mathbf{1}_{u(z)>\zeta}-\mathbf{1}_{0>\zeta}\right) \mathrm{d} \zeta .
$$

By (2.27), it follows that

$$
\int_{\mathbb{R}} H^{\prime}(\zeta)\left(f(z, \zeta)-\mathbf{1}_{u(z)>\zeta}\right) \mathrm{d} \zeta \geqq 0
$$

for all convex and sub-linear $H \in C^{1}(\mathbb{R})$. Approximating $\zeta \mapsto(\zeta-\xi)^{-}$by such functions $H$, we obtain $m(\xi) \geqq 0$. 


\subsection{Left Limits of Generalized Solutions}

If $(f(t))_{t \in[0, T]}$ is a generalized solution to (1.1) and $\varphi \in C_{c}^{\infty}\left(\mathbb{T}^{N} \times \mathbb{R}\right)$, then, a.s., $t \mapsto\langle f(t), \varphi\rangle$ is càdlàg. In the next proposition, we show that the a.s.-property to be càdlàg is independent on $\varphi$ and that the limit from the left at any point $t_{*} \in(0, T]$ is represented by a kinetic function.

Proposition 2.10. Let $f_{0}$ be a kinetic initial datum. Let $(f(t))_{t \in[0, T]}$ be a generalized solution to (1.1) with initial datum $f_{0}$. Then

1. there exists a measurable subset $\hat{\Omega} \subset \Omega$ of probability 1 such that, for all $\omega \in \hat{\Omega}$, for all $\varphi \in C_{c}\left(\mathbb{T}^{N} \times \mathbb{R}\right), t \mapsto\langle f(\omega, t), \varphi\rangle$ is càdlàg,

2. there exists an $L^{\infty}\left(\mathbb{T}^{N} \times \mathbb{R} ;[0,1]\right)$-valued process $\left(f^{-}(t)\right)_{t \in(0, T]}$ such that: for all $t \in(0, T]$, for all $\omega \in \hat{\Omega}$, for all $\varphi \in C_{c}\left(\mathbb{T}^{N} \times \mathbb{R}\right), f^{-}(t)$ is a kinetic function on $\mathbb{T}^{N}$ which represents the left limit of $s \mapsto\langle f(s), \varphi\rangle$ at $t$ :

$$
\left\langle f^{-}(t), \varphi\right\rangle=\lim _{s \rightarrow t-}\langle f(s), \varphi\rangle .
$$

Proof of Proposition 2.10. The set of test functions $C_{c}^{1}\left(\mathbb{T}^{N} \times \mathbb{R}\right)$ (endowed with the topology of the uniform convergence of the functions and their first derivatives) is separable and we fix a dense countable subset $\mathcal{D}_{1}$ (see the argument about $\Gamma$ in Section 4.5.1 for a proof of the existence of $\left.\mathcal{D}_{1}\right)$. For all $\varphi \in C_{c}^{1}\left(\mathbb{T}^{N} \times \mathbb{R}\right)$, a.s., the map

$$
\begin{aligned}
J_{\varphi} & : t \mapsto \int_{0}^{t}\langle f(s), a(\xi) \cdot \nabla \varphi\rangle \mathrm{d} s \\
& +\sum_{k \geqq 1} \int_{0}^{t} \int_{\mathbb{T}^{N}} \int_{\mathbb{R}} g_{k}(x, \xi) \varphi(x, \xi) \mathrm{d} v_{x, s}(\xi) \mathrm{d} x \mathrm{~d} \beta_{k}(s) \\
& +\frac{1}{2} \int_{0}^{t} \int_{\mathbb{T}^{N}} \int_{\mathbb{R}} \partial_{\xi} \varphi(x, \xi) \mathbf{G}^{2}(x, \xi) \mathrm{d} v_{x, s}(\xi) \mathrm{d} x \mathrm{~d} s
\end{aligned}
$$

is continuous on $[0, T]$. Consequently, a.s., say for $\omega \in \Omega_{1}$ where $\Omega_{1}$ is of full measure, for all $\varphi \in \mathcal{D}_{1}, J_{\varphi}$ is continuous on $[0, T]$. If $\varphi \in \mathcal{D}_{1}$, (2.23) gives $\langle f(t), \varphi\rangle$ as a sum (up to the constant $\left\langle f_{0}, \varphi\right\rangle$ ) of $J_{\varphi}(t)$ with $m\left(\partial_{\xi} \varphi\right)([0, t])$. This latter expression defines a function càdlàg in $t$ for all $\omega \in \Omega_{2}$, hence $t \mapsto\langle f(t), \varphi\rangle$ is càdlàg if $\omega \in \Omega_{1} \cap \Omega_{2}$. Here, $\Omega_{2} \subset \Omega$ is of full measure. Next, we use the estimate (2.22): there exists a set of full measure $\Omega_{3}$ in $\Omega$ such that, for every $\omega \in \Omega_{3}$,

$$
\sup _{t \in[0, T]} \int_{\mathbb{T}^{N}} \int_{\mathbb{R}}|\xi|^{p} \mathrm{~d} v_{x, t}(\xi) \mathrm{d} x \leqq C_{p}(\omega)<+\infty .
$$

Let $\omega \in \hat{\Omega}:=\Omega_{1} \cap \Omega_{2} \cap \Omega_{3}$ be fixed. If $\varphi \in C_{c}\left(\mathbb{T}^{N} \times \mathbb{R}\right)$, then

$$
\langle f(t), \varphi\rangle=\int_{\mathbb{T}^{N} \times \mathbb{R}} \psi(x, \xi) \mathrm{d} v_{x, t}(\xi) \mathrm{d} x, \quad \psi(x, \xi):=\int_{-\infty}^{\xi} \varphi(x, \zeta) \mathrm{d} \zeta .
$$


Let $R_{\varphi}>0$ be such that $\varphi$ is supported in $\left[-R_{\varphi}, R_{\varphi}\right]$. Since $|\psi(x, \xi)| \leqq\|\varphi\|_{L^{\infty}}\left(R_{\varphi}+\right.$ $|\xi|)$, we obtain the bound $\sup _{t \in[0, T]}|\langle f(t), \varphi\rangle| \leqq\|\varphi\|_{L^{\infty}}\left(R_{\varphi}+C_{1}(\omega)\right)$. This gives the continuity of $\langle f(t), \varphi\rangle$ with respect to $\varphi$. Since the space of càdlàg functions is closed under uniform convergence, an argument of density shows that $t \mapsto\langle f(t), \varphi\rangle$ is càdlàg for all $\varphi \in C_{c}\left(\mathbb{T}^{N} \times \mathbb{R}\right)$. To prove the second assertion of the proposition, let us fix $\omega \in \hat{\Omega}$ and consider an increasing sequence $\left(t_{n}\right)$ in $[0, T]$ converging to a point $t_{*} \in(0, T]$. Then, by means of (2.30) and since the Borel $\sigma$-algebra of $\mathbb{T}^{N}$ is countably generated ( $\mathbb{T}^{N}$ being separable), we can apply Corollary 2.5: there exist a kinetic function $f^{*,-}$ on $\mathbb{T}^{N} \times \mathbb{R}$ and a subsequence $\left(n_{k}\right)$ such that $f\left(t_{n_{k}}\right) \rightarrow f^{*,-}$ weakly-* in $L^{\infty}\left(\mathbb{T}^{N} \times \mathbb{R}\right)$ as $k \rightarrow+\infty$. If an other subsequence $\left(\tilde{n}_{k}\right)$ provides an other weak limit $\tilde{f}^{*,-}$, then we have

$$
\left\langle f^{*,-}, \varphi\right\rangle=\lim _{t \rightarrow t^{*}-}\langle f(t), \varphi\rangle=\left\langle\tilde{f}^{*,-}, \varphi\right\rangle
$$

for all $\varphi \in C_{c}\left(\mathbb{T}^{N} \times \mathbb{R}\right)$. Therefore $f^{*,-}=\tilde{f}^{*,-}$ : there is only one possible limit. It follows that the whole sequence $\left(f\left(t_{n}\right)\right)$ is converging to $f^{*,-}$ in $L^{\infty}\left(\mathbb{T}^{N} \times \mathbb{R}\right)$ weak-*. We establish this fact to ensure that the subsequence $\left(n_{k}\right)$ is independent on $\omega$. Indeed, this shows that, viewed as a function of $(\omega, x, \xi), f^{*,-}$ is measurable. We set $f^{-}\left(t_{*}\right)=f^{*,-}$ to conclude.

Remark 2.2. (Left and right limits). Note that we prove a little bit more than what is stated in Proposition 2.10. Indeed, for $\omega \in \hat{\Omega}$, we have $f(s) \rightarrow f^{-}(t)$ in $L^{\infty}\left(\mathbb{T}^{N} \times \mathbb{R}\right)$ for the weak-* topology, when $s \uparrow t$, which implies (2.28). By similar arguments, we can show that $f(s) \rightarrow f(t)$ in $L^{\infty}\left(\mathbb{T}^{N} \times \mathbb{R}\right)$ weak-* when $s \downarrow t$.

Remark 2.3. (Uniform bound). Note that, by construction, $v^{-}=-\partial_{\xi} f^{-}$satisfies the following bounds: for all $\omega \in \hat{\Omega}$,

$$
\sup _{t \in[0, T]} \int_{\mathbb{T}^{N}} \int_{\mathbb{R}}|\xi|^{p} \mathrm{~d} \nu_{x, t}^{-}(\xi) \mathrm{d} x \leqq C_{p}(\omega), \quad \mathbb{E}\left(\sup _{t \in[0, T]} \int_{\mathbb{T}^{N}} \int_{\mathbb{R}}|\xi|^{p} \mathrm{~d} v_{x, t}^{-}(\xi) \mathrm{d} x\right) \leqq C_{p}
$$

We obtain (2.32) using (2.22)-(2.30) and Fatou's Lemma.

Remark 2.4. (Equation for $f^{-}$). Passing to the limit in (2.23) for an increasing sequence of times $t$, we obtain the following equation on $f^{-}$:

$$
\begin{aligned}
\left\langle f^{-}(t), \varphi\right\rangle= & \langle f(0), \varphi\rangle+\int_{0}^{t}\left\langle f(s), a(\xi) \cdot \nabla_{x} \varphi\right\rangle \mathrm{d} s \\
& +\int_{0}^{t} \int_{\mathbb{T}^{N}} \int_{\mathbb{R}} g_{k}(x, \xi) \varphi(x, \xi) \mathrm{d} v_{x, s}(\xi) \mathrm{d} x \mathrm{~d} \beta_{k}(s) \\
& +\frac{1}{2} \int_{0}^{t} \int_{\mathbb{T}^{N}} \int_{\mathbb{R}} \mathbf{G}^{2}(x, \xi) \partial_{\xi} \varphi(x, \xi) \mathrm{d} v_{x, s}(\xi) \mathrm{d} x \mathrm{~d} s-m\left(\partial_{\xi} \varphi\right)([0, t)) .
\end{aligned}
$$


In particular, we have

$$
\left\langle f(t)-f^{-}(t), \varphi\right\rangle=-m\left(\partial_{\xi} \varphi\right)(\{t\}) .
$$

Outside the set of atomic points of $A \mapsto m\left(\partial_{\xi} \varphi\right)(A)$, which is at most countable, we have $\langle f(t), \varphi\rangle=\left\langle f^{-}(t), \varphi\right\rangle$. It follows that $f=f^{-}$almost everywhere. In particular, (2.33) gives us the following equation on $f^{-}$:

$$
\begin{aligned}
\left\langle f^{-}(t), \varphi\right\rangle= & \langle f(0), \varphi\rangle+\int_{0}^{t}\left\langle f^{-}(s), a(\xi) \cdot \nabla_{x} \varphi\right\rangle \mathrm{d} s \\
& +\int_{0}^{t} \int_{\mathbb{T}^{N}} \int_{\mathbb{R}} g_{k}(x, \xi) \varphi(x, \xi) \mathrm{d} v_{x, s}^{-}(\xi) \mathrm{d} x \mathrm{~d} \beta_{k}(s) \\
& +\frac{1}{2} \int_{0}^{t} \int_{\mathbb{T}^{N}} \int_{\mathbb{R}} \mathbf{G}^{2}(x, \xi) \partial_{\xi} \varphi(x, \xi) \mathrm{d} v_{x, s}^{-}(\xi) \mathrm{d} x \mathrm{~d} s-m\left(\partial_{\xi} \varphi\right)([0, t)),
\end{aligned}
$$

an equation which is also valid for $t=0$ if we set $f^{-}(0)=f_{0}$.

In the next proposition, we give a criterion for the continuity of $t \mapsto\langle f(t), \varphi\rangle$ at a given point.

Proposition 2.11. (The case of equilibrium). Let $f_{0}$ be a kinetic initial datum. Let $(f(t))_{t \in[0, T]}$ be a generalized solution to $(1.1)$ with initial datum $f_{0}$. Let $t \in(0, T]$. Assume that $f^{-}(t)$ is at equilibrium: there exists a random variable $v \in L^{1}\left(\mathbb{T}^{N}\right)$ such that $f^{-}(t, \xi)=\mathbf{1}_{v>\xi}$ a.s. Then $f^{-}(t)=f(t)$.

Proof of Proposition 2.11. Let $m^{*}$ denote the restriction of $m$ to $\mathbb{T}^{N} \times\{t\} \times \mathbb{R}$. Let us also set $f^{+}=f(t)$. By (2.34), we thus have

$$
f^{+}-\mathbf{1}_{v>\xi}=\partial_{\xi} m^{*} .
$$

There exists a subset $\Omega_{4}$ of $\Omega$ of probability 1 such that, for all $\omega \in \Omega_{4}, m$, and thus $m^{*}$, are finite measures on $\mathbb{T}^{N} \times[0, T] \times \mathbb{R}$ and $\mathbb{T}^{N} \times \mathbb{R}$ respectively. Let $\psi$ be a smooth non-negative function such that $0 \leqq \psi \leqq 1, \psi \equiv 1$ on $[-1,1], \psi$ being supported in $[-2,2]$. Define the cut-off function $\psi_{\varepsilon}(\xi)=\psi(\varepsilon \xi)$. Let also $\varphi \in C\left(\mathbb{T}^{N}\right)$. By (2.36), we have

$$
\begin{aligned}
& \iint_{\mathbb{T}^{N} \times \mathbb{R}}\left(f^{+}(x, \xi)-\mathbf{1}_{v(x)>\xi}\right) \varphi(x) \psi_{\varepsilon}(\xi) \mathrm{d} x \mathrm{~d} \xi \\
& \quad=-\varepsilon \iint_{\mathbb{T}^{N} \times \mathbb{R}} \varphi(x) \psi^{\prime}(\varepsilon \xi) d m^{*} \\
& \quad \leqq \varepsilon\|\varphi\|_{L^{\infty}\left(\mathbb{T}^{N}\right)}\left\|\psi^{\prime}\right\|_{L^{\infty}(\mathbb{R})} m^{*}\left(\mathbb{T}^{N} \times \mathbb{R}\right) .
\end{aligned}
$$

Taking the limit $\varepsilon \rightarrow 0$, and taking into consideration the fact that $\varphi$ is arbitrary, we deduce that, for all $\omega \in \hat{\Omega} \cap \Omega_{4}$, for almost every $x \in \mathbb{T}^{N}$,

$$
\int_{\mathbb{R}}\left(f^{+}(x, \xi)-\mathbf{1}_{0>\xi}\right) \mathrm{d} \xi=\int_{\mathbb{R}}\left(\mathbf{1}_{v(x)>\xi}-\mathbf{1}_{0>\xi}\right) \mathrm{d} \xi=v(x) .
$$


Now introduce

$$
p^{*}: \xi \mapsto \int_{-\infty}^{\xi}\left(\mathbf{1}_{v>\zeta}-f^{+}(\zeta)\right) \mathrm{d} \zeta .
$$

By Lemma 2.9, $p^{*}$ is non-negative. In addition, $\partial \xi\left(m^{*}+p^{*}\right)=0$, due to (2.36) and the definition of $p^{*}$. Therefore $m^{*}+p^{*}$ is constant, and actually vanishes by the condition at infinity (2.3) and the obvious fact that $p^{*}\left(\mathbb{T}^{N} \times B_{R}^{c}\right)$ also vanishes when $R \rightarrow+\infty$. Since $m^{*}, p^{*} \geqq 0$, we finally obtain $m^{*}=0$ and conclude with the identity $f^{-}(t)=f(t)$.

Let us consider also the special case $t=0$. By letting $t \rightarrow 0+$ in (2.23), we have $f(0)-f_{0}=\partial_{\xi} m_{0}$, where $m_{0}$ is the restriction of $m$ to $\mathbb{T}^{N} \times\{0\} \times \mathbb{R}$. Consequently, we have the following corollary to Proposition 2.11.

Corollary 2.12. Let $f_{0}$ be a kinetic initial datum. Let $(f(t))_{t \in[0, T]}$ be a generalized solution to (1.1) with initial datum $f_{0}$. Assume that $f_{0}$ is at equilibrium. Then $f(0)=f_{0}$ and $m$ does not charge the line $\{t=0\}: m\left(\mathbb{T}^{N} \times\{0\} \times \mathbb{R}\right)=0$ a.s..

Our final result in this section is about trajectories of solutions to (1.1). It is an intermediate statement, before the full continuity result given in Corollary 3.3.

Proposition 2.13. Let $u_{0} \in L^{\infty}\left(\mathbb{T}^{N}\right)$. Let $(u(t))_{t \in[0, T]}$ be a solution to (1.1) with initial datum $u_{0}$. Then, for all $p \in[1,+\infty$ ), for all $\omega \in \hat{\Omega}$ (given in Proposition 2.10), the map $t \mapsto u(t)$ from $[0, T]$ to $L^{p}\left(\mathbb{T}^{N}\right)$ is continuous from the right.

Proof of Proposition 2.13. We apply Proposition 2.10 to $f(t)=f(t)=\mathbf{1}_{u(t)>\xi}$. For $\omega \in \hat{\Omega}, \varphi \in C_{c}\left(\mathbb{T}^{N} \times \mathbb{R}\right)$, the map $t \mapsto\langle\mathrm{f}(t), \varphi\rangle$ is càdlàg. Let $t_{*} \in[0, T)$ and let $\left(t_{n}\right)$ be a decreasing sequence of $[0, T]$ converging to $t_{*}$. The sequence $f^{n}$ of elements $f^{n}:=\mathrm{f}\left(t_{n}\right)$ takes values in $[0,1]$. For $\omega \in \hat{\Omega}$ fixed, it has a convergent subsequence in $L^{\infty}\left(\mathbb{T}^{N} \times \mathbb{R}\right)$ weak-*. Since $\left\langle f^{n}, \varphi\right\rangle \rightarrow\left\langle\mathrm{f}\left(t_{*}\right), \varphi\right\rangle$ for all continuous, compactly supported function $\varphi$ on $\mathbb{T}^{N} \times \mathbb{R}$, the whole sequence $\left(f^{n}\right)$ is converging to its unique adherence value, $\mathrm{f}\left(t_{*}\right)$. By (2.5), the bound (2.14) is satisfied for all $p \in[1,+\infty)$; we can apply Lemma 2.6 to conclude with the convergence $u\left(t_{n}\right) \rightarrow u\left(t_{*}\right)$ in $L^{p}\left(\mathbb{T}^{N}\right)$.

\section{Comparison, Uniqueness and Reduction of Generalized Solutions}

\subsection{Doubling of Variables}

In this paragraph, we prove a technical proposition relating two generalized solutions $f_{i}, i=1,2$ of the equation

$$
\mathrm{d} u_{i}(x, t)+\operatorname{div}\left(A\left(u_{i}(x, t)\right)\right) \mathrm{d} t=\Phi_{i}\left(x, u_{i}(x, t)\right) \mathrm{d} W(t) .
$$

We use the following convention of notations: if $(f(t))_{t \in[0, T]}$ is a generalized solution to (1.1), we denote by $f^{-}$the left limit defined in Proposition 2.10, and we denote by $f^{+}$the right limit, which is simply $f: f^{+}(t):=f(t)$. This gives more homogeneity to the different statements in this part. Recall also the notation $\bar{f}=1-f$ for the conjugate to $f$, introduced in Definition 2.5. 
Proposition 3.1. Let $f_{i}, i=1,2$, be generalized solution to (3.1). Then, for $0 \leqq$ $t \leqq T$, and non-negative test functions $\rho \in C^{\infty}\left(\mathbb{T}^{N}\right), \psi \in C_{c}^{\infty}(\mathbb{R})$, we have

$$
\begin{aligned}
& \mathbb{E} \int_{\left(\mathbb{T}^{N}\right)^{2}} \int_{\mathbb{R}^{2}} \rho(x-y) \psi(\xi-\zeta) f_{1}^{ \pm}(x, t, \xi) \bar{f}_{2}^{ \pm}(y, t, \zeta) \mathrm{d} \xi \mathrm{d} \zeta \mathrm{d} x \mathrm{~d} y \\
& \quad \leqq \int_{\left(\mathbb{T}^{N}\right)^{2}} \int_{\mathbb{R}^{2}} \rho(x-y) \psi(\xi-\zeta) f_{1,0}(x, \xi) \bar{f}_{2,0}(y, \zeta) \mathrm{d} \xi \mathrm{d} \zeta \mathrm{d} x \mathrm{~d} y+\mathrm{I}_{\rho}+\mathrm{I}_{\psi}
\end{aligned}
$$

where

$$
\begin{aligned}
\mathrm{I}_{\rho}= & \mathbb{E} \int_{0}^{t} \int_{\left(\mathbb{T}^{N}\right)^{2}} \int_{\mathbb{R}^{2}} f_{1}(x, s, \xi) \bar{f}_{2}(y, s, \zeta)(a(\xi) \\
& -a(\zeta)) \psi(\xi-\zeta) \mathrm{d} \xi \mathrm{d} \zeta \cdot \nabla_{x} \rho(x-y) \mathrm{d} x \mathrm{~d} y \mathrm{~d} s
\end{aligned}
$$

and

$$
\begin{aligned}
\mathrm{I}_{\psi}= & \frac{1}{2} \int_{\left(\mathbb{T}^{N}\right)^{2}} \rho(x-y) \mathbb{E} \int_{0}^{t} \int_{\mathbb{R}^{2}} \psi(\xi-\zeta) \sum_{k \geqq 1} \mid g_{k, 1}(x, \xi) \\
& -\left.g_{k, 2}(y, \zeta)\right|^{2} \mathrm{~d} v_{x, s}^{1} \otimes v_{y, s}^{2}(\xi, \zeta) \mathrm{d} x \mathrm{~d} y \mathrm{~d} s .
\end{aligned}
$$

Remark 3.1. Each term in (3.2) is finite. Let us consider the left-hand side of (3.2). Introduce the auxiliary functions

$$
\psi_{1}(\xi)=\int_{-\infty}^{\xi} \psi(s) \mathrm{d} s, \quad \psi_{2}(\zeta)=\int_{-\infty}^{\zeta} \psi_{1}(\xi) \mathrm{d} \xi .
$$

Since $\psi$ is compactly supported, both $\psi_{1}$ and $\psi_{2}$ vanish at $-\infty$. When $\xi \rightarrow+\infty$, $\psi_{1}$ remains bounded while $\psi_{2}$ has linear growth. More precisely, if $\psi$ is supported in $[-R, R]$, then

$$
\left|\psi_{2}(\zeta)\right| \leqq(|\zeta|+R)\|\psi\|_{L^{1}(\mathbb{R})}
$$

Since

$$
f_{1}^{ \pm}(x, t, \xi)=\int_{(\xi,+\infty)} \mathrm{d} v_{x, t}^{1, \pm}(\xi), \quad \bar{f}_{2}^{ \pm}(y, t, \zeta)=\int_{(-\infty, \zeta)} \mathrm{d} v_{y, t}^{2, \pm}(\zeta),
$$

for almost every $\xi, \zeta \in \mathbb{R}, x, y \in \mathbb{T}^{N}, t \in[0, T]$, the Fubini Theorem gives us the formula

$$
\int_{\mathbb{R}^{2}} \psi(\xi-\zeta) f_{1}^{ \pm}(x, t, \xi) \bar{f}_{2}^{ \pm}(y, t, \zeta) \mathrm{d} \xi \mathrm{d} \zeta=\int_{\mathbb{R}^{2}} \psi_{2}(u-v) \mathrm{d} v_{x, t}^{1, \pm}(u) \mathrm{d} v_{y, t}^{2, \pm}(v) .
$$

By (3.3), we deduce that

$$
\begin{aligned}
& \left|\int_{\mathbb{R}^{2}} \psi(\xi-\zeta) f_{1}^{ \pm}(x, t, \xi) \bar{f}_{2}^{ \pm}(y, t, \zeta) \mathrm{d} \xi \mathrm{d} \zeta\right| \\
& \quad \leqq\|\psi\|_{L^{1}(\mathbb{R})}\left[R+\int_{\mathbb{R}}|\xi| \mathrm{d} \nu_{x, t}^{1, \pm}(\xi)+\int_{\mathbb{R}}|\xi| \mathrm{d} \nu_{y, t}^{2, \pm}(\xi)\right]
\end{aligned}
$$


for almost every $x, y \in \mathbb{T}^{N}$, for all $t \in[0, T]$. Using the Young inequality for convolution with indices $1,1,1$, we obtain

$$
\begin{aligned}
& \left|\int_{\left(\mathbb{T}^{N}\right)^{2}} \int_{\mathbb{R}^{2}} \rho(x-y) \psi(\xi-\zeta) f_{1}^{ \pm}(x, t, \xi) \bar{f}_{2}^{ \pm}(y, t, \zeta) \mathrm{d} \xi \mathrm{d} \zeta \mathrm{d} x \mathrm{~d} y\right| \\
& \quad \leqq\|\psi\|_{L^{1}(\mathbb{R})}\|\rho\|_{L^{1}\left(\mathbb{T}^{N}\right)}\left(R+C_{1,1}(\omega)+C_{1,2}(\omega)\right),
\end{aligned}
$$

where

$$
C_{1, i}(\omega):=\sup _{t \in[0, T]} \int_{\mathbb{T}^{N}} \int_{\mathbb{R}}|\xi| \mathrm{d} \nu_{x, t}^{i, \pm}(\xi) \mathrm{d} x
$$

is in $L^{1}(\Omega)$ thanks to (2.22)-(2.32).

Proof of Proposition 3.1. Set

$$
G_{i}^{2}(x, \xi)=\sum_{k=1}^{\infty}\left|g_{k, i}(x, \xi)\right|^{2}, \quad i \in\{1,2\}
$$

Let $\varphi_{1} \in C_{c}^{\infty}\left(\mathbb{T}_{x}^{N} \times \mathbb{R}_{\xi}\right)$ and $\varphi_{2} \in C_{c}^{\infty}\left(\mathbb{T}_{y}^{N} \times \mathbb{R}_{\zeta}\right)$ be some given test functions. Equation (2.23) for $f_{1}=f_{1}^{+}$reads $\left\langle f_{1}^{+}(t), \varphi_{1}\right\rangle=\mu_{1}([0, t])+F_{1}(t)$, where $F_{1}$ is the stochastic integral

$$
F_{1}(t)=\sum_{k \geqq 1} \int_{0}^{t} \int_{\mathbb{T}^{N}} \int_{\mathbb{R}} g_{k, 1} \varphi_{1} \mathrm{~d} \nu_{x, s}^{1}(\xi) \mathrm{d} x \mathrm{~d} \beta_{k}(s)
$$

and $t \mapsto \mu_{1}([0, t])$ is the function of finite variation on $[0, T](c f .[42$, p. 5]) defined by

$$
\begin{aligned}
\mu_{1}([0, t])= & \left\langle f_{1,0}, \varphi_{1}\right\rangle \delta_{0}([0, t])+\int_{0}^{t}\left\langle f_{1}, a \cdot \nabla \varphi_{1}\right\rangle \mathrm{d} s \\
& +\frac{1}{2} \int_{0}^{t} \int_{\mathbb{T}^{N}} \int_{\mathbb{R}} \partial_{\xi} \varphi_{1} \mathbf{G}_{1}^{2} \mathrm{~d} v_{x, s}^{1}(\xi) \mathrm{d} x \mathrm{~d} s-m_{1}\left(\partial_{\xi} \varphi_{1}\right)([0, t]) .
\end{aligned}
$$

Note that, by Corollary 2.12, $m_{1}\left(\partial_{\xi} \varphi_{1}\right)(\{0\})=0$ and thus the value of $\mu_{1}(\{0\})$ is $\left\langle f_{1,0}, \varphi_{1}\right\rangle$. Similarly, we write $\left\langle\bar{f}_{2}^{+}(t), \varphi_{2}\right\rangle$ as continuous semimartingale, sum of the stochastic integral

$$
\bar{F}_{2}(t)=-\sum_{k \geqq 1} \int_{0}^{t} \int_{\mathbb{T}^{N}} \int_{\mathbb{R}} g_{k, 2} \varphi_{2} \mathrm{~d} \nu_{y, s}^{2}(\zeta) \mathrm{d} y \mathrm{~d} \beta_{k}(s)
$$

with the function with finite variation given by

$$
\begin{aligned}
\mu_{2}([0, t])= & \left\langle\bar{f}_{2,0}, \varphi_{2}\right\rangle \delta_{0}([0, t])+\int_{0}^{t}\left\langle\bar{f}_{2}, a \cdot \nabla \varphi_{2}\right\rangle \mathrm{d} s \\
& -\frac{1}{2} \int_{0}^{t} \int_{\mathbb{T}^{N}} \int_{\mathbb{R}} \partial_{\xi} \varphi_{2} \mathbf{G}_{2}^{2} \mathrm{~d} v_{y, s}^{2}(\zeta) \mathrm{d} y \mathrm{~d} s+m_{2}\left(\partial_{\zeta} \varphi_{2}\right)([0, t]) .
\end{aligned}
$$


Again, we note that $\mu_{2}(\{0\})=\left\langle\bar{f}_{2,0}, \varphi_{2}\right\rangle$. Let us define the test-function

$$
\alpha(x, \xi, y, \zeta)=\varphi_{1}(x, \xi) \varphi_{2}(y, \zeta) .
$$

We want to compute

$$
\left\langle\left\langle f_{1}^{+}(t) \bar{f}_{2}^{+}(t), \alpha\right\rangle\right\rangle=\left\langle f_{1}^{+}(t), \varphi_{1}\right\rangle\left\langle\bar{f}_{2}^{+}(t), \varphi_{2}\right\rangle,
$$

where $\langle\langle\cdot, \cdot\rangle\rangle$ denotes the duality product over $\mathbb{T}_{x}^{N} \times \mathbb{R}_{\xi} \times \mathbb{T}_{y}^{N} \times \mathbb{R}_{\zeta}$. By the Itō formula for continuous semimartingales, [42, p. 146], taking expectation, we obtain the following identity:

$$
\begin{aligned}
\mathbb{E}\left\langle\left\langle f_{1}^{+}(t) \bar{f}_{2}^{+}(t), \alpha\right\rangle\right\rangle= & \left\langle\left\langle f_{1,0} \bar{f}_{2,0}, \alpha\right\rangle\right\rangle \\
& +\mathbb{E} \int_{0}^{t} \int_{\left(\mathbb{T}^{N}\right)^{2}} \int_{\mathbb{R}^{2}} f_{1} \bar{f}_{2}\left(a(\xi) \cdot \nabla_{x}\right. \\
& \left.+a(\zeta) \cdot \nabla_{y}\right) \alpha \mathrm{d} \xi \mathrm{d} \zeta \mathrm{d} x \mathrm{~d} y \mathrm{~d} s \\
& +\frac{1}{2} \mathbb{E} \int_{0}^{t} \int_{\left(\mathbb{T}^{N}\right)^{2}} \int_{\mathbb{R}^{2}} \partial_{\xi} \alpha \bar{f}_{2}(s) \mathbf{G}_{1}^{2} \mathrm{~d} \nu_{x, s}^{1}(\xi) \mathrm{d} \zeta \mathrm{d} x \mathrm{~d} y \mathrm{~d} s \\
& -\frac{1}{2} \mathbb{E} \int_{0}^{t} \int_{\left(\mathbb{T}^{N}\right)^{2}} \int_{\mathbb{R}^{2}} \partial_{\zeta} \alpha f_{1}(s) \mathbf{G}_{2}^{2} \mathrm{~d} \nu_{y, s}^{2}(\zeta) \mathrm{d} \xi \mathrm{d} y \mathrm{~d} x \mathrm{~d} s \\
& -\mathbb{E} \int_{0}^{t} \int_{\left(\mathbb{T}^{N}\right)^{2}} \int_{\mathbb{R}^{2}} \mathbf{G}_{1,2} \alpha \mathrm{d} \nu_{x, s}^{1}(\xi) \mathrm{d} v_{y, s}^{2}(\zeta) \mathrm{d} x \mathrm{~d} y \mathrm{~d} s \\
& -\mathbb{E} \int_{(0, t]} \int_{\left(\mathbb{T}^{N}\right)^{2}} \int_{\mathbb{R}^{2}} \bar{f}_{2}^{+}(s) \partial_{\xi} \alpha \mathrm{d} m_{1}(x, s, \xi) \mathrm{d} \zeta \mathrm{d} y \\
& +\mathbb{E} \int_{(0, t]} \int_{\left(\mathbb{T}^{N}\right)^{2}} \int_{\mathbb{R}^{2}} f_{1}^{-}(s) \partial_{\zeta} \alpha \mathrm{d} m_{2}(y, s, \zeta) \mathrm{d} \xi \mathrm{d} x,
\end{aligned}
$$

where $\mathbf{G}_{1,2}(x, y ; \xi, \zeta):=\sum_{k \geqq 1} g_{k, 1}(x, \xi) g_{k, 2}(y, \zeta)$. Equation (3.7) is identical to Equation (24) in [14]. We refer to the proof of Proposition 9 in [14] for the last arguments that establish (3.2) on the basis of (3.7).

\subsection{Uniqueness, Reduction of Generalized Solution}

In this section we use Proposition 3.1 above to deduce the uniqueness of solutions and the reduction of generalized solutions to solutions.

Theorem 3.2. (Uniqueness, reduction). Let $u_{0} \in L^{\infty}\left(\mathbb{T}^{N}\right)$. Assume (1.3)-(1.4). Then we have the following results:

1. there is at most one solution with initial datum $u_{0}$ to (1.1);

2. If $f$ is a generalized solution to (1.1) with initial datum $f_{0}$ at equilibrium: $f_{0}=$ $\mathbf{1}_{u_{0}>\xi}$, then there exists a solution $u$ to (1.1) with initial datum $u_{0}$ such that $f(x, t, \xi)=\mathbf{1}_{u(x, t)>\xi}$ a.s., for almost every $(x, t, \xi)$; 
3. if $u_{1}, u_{2}$ are two solutions to (1.1) associated with the initial data $u_{1,0}, u_{2,0} \in$ $L^{\infty}\left(\mathbb{T}^{N}\right)$ respectively, then

$$
\mathbb{E}\left\|\left(u_{1}(t)-u_{2}(t)\right)^{+}\right\|_{L^{1}\left(\mathbb{T}^{N}\right)} \leqq\left\|\left(u_{1,0}-u_{2,0}\right)^{+}\right\|_{L^{1}\left(\mathbb{T}^{N}\right)},
$$

for all $t \in[0, T]$. This implies the $L^{1}$-contraction property, and the comparison principle for solutions.

Corollary 3.3. (Continuity in time). Let $u_{0} \in L^{\infty}\left(\mathbb{T}^{N}\right)$. Assume (1.3)-(1.4). Then, for every $p \in[1,+\infty)$, the solution $u$ to (1.1) with initial datum $u_{0}$ has a representative in $L^{p}\left(\Omega ; L^{\infty}\left(0, T ; L^{p}\left(\mathbb{T}^{N}\right)\right)\right)$ with almost sure continuous trajectories in $L^{p}\left(\mathbb{T}^{N}\right)$.

Remark 3.2. (Uniqueness of the kinetic measure). Let $f$ and $\check{f}$ be two generalized solution to (1.1) with initial datum $f_{0}$ at equilibrium, $f_{0}=\mathbf{1}_{u_{0}>\xi}$. By Theorem 3.2, we have $f=\check{f}$. It follows from (2.23) that the associated random measures $m$ and $\check{m}$ satisfy, for all $\varphi \in C_{c}^{1}\left(\mathbb{T}^{N} \times \mathbb{R}\right)$, and for all $t \in[0, T]$, almost surely,

$$
m\left(\partial_{\xi} \varphi\right)([0, t])=\check{m}\left(\partial_{\xi} \varphi\right)([0, t]) .
$$

At fixed $\varphi$, the two functions of $t$ in (3.9) are càdlàg. Therefore (3.9) is satisfied for all $\varphi \in C_{c}^{1}\left(\mathbb{T}^{N} \times \mathbb{R}\right)$, almost surely, for all $t \in[0, T]$. By an argument of density (as in the proof of Proposition 2.10), we obtain (3.9) almost surely, for all $\varphi \in C_{c}^{1}\left(\mathbb{T}^{N} \times \mathbb{R}\right)$, for all $t \in[0, T]$. This implies: almost surely, $\partial_{\xi} m=\partial_{\xi} \check{m}$. By (2.10), the two measures have the same total mass almost surely. Consequently, almost surely, $m=\check{m}$.

Proof of Theorem 3.2. Consider first the additive case: $\Phi(x, u(x))$ independent on $u(x)$. Let $f_{i}, i=1,2$ be two generalized solutions to (1.1). Then, we use (3.2) with $g_{k}$ independent on $\xi$ and $\zeta$. By (1.4), the last term $\mathrm{I}_{\psi}$ is bounded by

$$
\frac{t D_{1}}{2}\|\psi\|_{L^{\infty}} \int_{\left(\mathbb{T}^{N}\right)^{2}}|x-y|^{2} \rho(x-y) \mathrm{d} x \mathrm{~d} y .
$$

We then take $\psi:=\psi_{\delta}$ and $\rho=\rho_{\varepsilon}$ where $\left(\psi_{\delta}\right)$ and $\left(\rho_{\varepsilon}\right)$ are approximations of the identity on $\mathbb{R}$ and $\mathbb{T}^{N}$, respectively, that is,

$$
\psi_{\delta}(\xi)=\frac{1}{\delta} \psi\left(\frac{\xi}{\delta}\right), \quad \rho_{\varepsilon}(x)=\frac{1}{\varepsilon^{N}} \rho\left(\frac{x}{\varepsilon}\right),
$$

where $\psi$ and $\rho$ are some given smooth probability densities on $\mathbb{R}$ and $\mathbb{T}^{N}$, respectively, to obtain

$$
\mathrm{I}_{\psi} \leqq \frac{t D_{1}}{2} \varepsilon^{2} \delta^{-1}
$$

Denote by $v_{x, t}^{i, \pm}$ the Young measures associated to $f_{i}^{ \pm}, i \in\{1,2\}$. By a computation similar to (3.4), we have, almost surely, for almost all $x, y \in \mathbb{T}^{N}$,

$$
\int_{\mathbb{R}} f_{1}^{ \pm}(x, t, \xi) \bar{f}_{2}^{ \pm}(y, t, \xi) \mathrm{d} \xi=\int_{\mathbb{R}^{2}}(u-v)^{+} \mathrm{d} v_{x, t}^{1, \pm}(u) \mathrm{d} v_{y, t}^{2, \pm}(v) .
$$


By (3.4), we also have

$\int_{\mathbb{R}^{2}} \psi_{\delta}(\xi-\zeta) f_{1}^{ \pm}(x, t, \xi) \bar{f}_{2}^{ \pm}(y, t, \zeta) \mathrm{d} \xi \mathrm{d} \zeta=\int_{\mathbb{R}^{2}} \psi_{2, \delta}(u-v) \mathrm{d} v_{x, t}^{1, \pm}(u) \mathrm{d} v_{y, t}^{2, \pm}(v)$,

where

$$
\psi_{2, \delta}(\xi)=\int_{-\infty}^{\xi} \psi_{1, \delta}(\zeta) \mathrm{d} \zeta, \quad \psi_{1, \delta}(\xi)=\int_{-\infty}^{\xi} \psi_{\delta}(\zeta) \mathrm{d} \zeta
$$

Assume that $\psi$ is supported in $(0,1)$. Then $\psi_{2, \delta}(\xi)=0$ if $\xi \leqq 0$ and, for $\xi>0$,

$$
\xi^{+}-\psi_{2, \delta}(\xi)=\int_{0}^{\xi^{+}} \int_{\zeta / \delta}^{+\infty} \psi(u) \mathrm{d} u \mathrm{~d} \zeta=\int_{0}^{+\infty} \xi^{+} \wedge(\delta u) \psi(u) \mathrm{d} u .
$$

Using (3.13) in (3.11), (3.12) gives

$$
\begin{aligned}
0 & \leqq \int_{\mathbb{R}} f_{1}^{ \pm}(x, t, \xi) \bar{f}_{2}^{ \pm}(y, t, \xi) \mathrm{d} \xi-\int_{\mathbb{R}^{2}} \psi_{\delta}(\xi-\zeta) f_{1}^{ \pm}(x, t, \xi) \bar{f}_{2}^{ \pm}(y, t, \zeta) \mathrm{d} \xi \mathrm{d} \zeta \\
& \leqq \int_{\mathbb{R}^{2}} \int_{0}^{+\infty}(u-v)^{+} \wedge(\delta \zeta) \psi(\zeta) \mathrm{d} \zeta \mathrm{d} \nu_{x, t}^{1, \pm}(u) \mathrm{d} v_{y, t}^{2, \pm}(v) .
\end{aligned}
$$

Since $(u-v)^{+} \wedge(\delta \zeta) \leqq|u| \wedge(\delta \zeta)+|v| \wedge(\delta \zeta)$, we have

$$
\begin{aligned}
0 & \leqq \int_{\mathbb{R}} f_{1}^{ \pm}(x, t, \xi) \bar{f}_{2}^{ \pm}(y, t, \xi) \mathrm{d} \xi-\int_{\mathbb{R}^{2}} \psi_{\delta}(\xi-\zeta) f_{1}^{ \pm}(x, t, \xi) \bar{f}_{2}^{ \pm}(y, t, \zeta) \mathrm{d} \xi \mathrm{d} \zeta \\
& \leqq \int_{0}^{+\infty}\left(\int_{\mathbb{R}}|\xi| \mathrm{d} \nu_{x, t}^{1, \pm}(\xi)+\int_{\mathbb{R}}|\xi| \mathrm{d} \nu_{y, t}^{2, \pm}(\xi)\right) \wedge(\delta \zeta) \psi(\zeta) \mathrm{d} \zeta .
\end{aligned}
$$

It follows that

$$
\begin{aligned}
& \mid \int_{\left(\mathbb{T}^{N}\right)^{2}} \int_{\mathbb{R}} \rho_{\varepsilon}(x-y) f_{1}^{ \pm}(x, t, \xi) \bar{f}_{2}^{ \pm}(y, t, \xi) \mathrm{d} \xi \mathrm{d} x \mathrm{~d} y \\
& \quad-\int_{\left(\mathbb{T}^{N}\right)^{2}} \int_{\mathbb{R}^{2}} \rho_{\varepsilon}(x-y) \psi_{\delta}(\xi-\zeta) f_{1}^{ \pm}(x, t, \xi) \bar{f}_{2}^{ \pm}(y, t, \zeta) \mathrm{d} \xi \mathrm{d} \zeta \mathrm{d} x \mathrm{~d} y \mid \\
& \quad \leqq \int_{0}^{+\infty}\left(\int_{\mathbb{T}^{N}} \int_{\mathbb{R}}|\xi|\left(\mathrm{d} \nu_{x, t}^{1, \pm}(\xi)+\mathrm{d} v_{x, t}^{2, \pm}(\xi)\right) \mathrm{d} x\right) \wedge(2 \delta \zeta) \psi(\zeta) \mathrm{d} \zeta \\
& \quad \leqq \int_{0}^{+\infty}\left(C_{1,1}^{ \pm}(\omega)+C_{1,2}^{ \pm}(\omega)\right) \wedge(2 \delta \zeta) \psi(\zeta) \mathrm{d} \zeta .
\end{aligned}
$$


We have used (2.22)-(2.32) (with a constant $C_{1, i}^{ \pm}$for $v^{i, \pm}$ ) to obtain (3.14). When $\varepsilon \rightarrow 0$, we have

$$
\begin{aligned}
& \mid \int_{\left(\mathbb{T}^{N}\right)^{2}} \int_{\mathbb{R}} \rho_{\varepsilon}(x-y) f_{1}^{ \pm}(x, t, \xi) \bar{f}_{2}^{ \pm}(y, t, \xi) \mathrm{d} \xi \mathrm{d} x \mathrm{~d} y \\
& \quad-\int_{\mathbb{T}^{N}} \int_{\mathbb{R}} f_{1}^{ \pm}(x, t, \xi) \bar{f}_{2}^{ \pm}(x, t, \xi) \mathrm{d} \xi \mathrm{d} x \mid \\
& \quad \leqq \sup _{|z|<\varepsilon} \int_{\mathbb{T}^{N}} \int_{\mathbb{R}} f_{1}^{ \pm}(x, t, \xi)\left|\bar{f}_{2}^{ \pm}(x-z, t, \xi)-\bar{f}_{2}^{ \pm}(x, t, \xi)\right| \mathrm{d} \xi \mathrm{d} x \\
& \quad \leqq \sup _{|z|<\varepsilon} \int_{\mathbb{T}^{N}} \int_{\mathbb{R}}\left|\chi_{f_{2}^{ \pm}}(x-z, t, \xi)-\chi_{f_{2}^{ \pm}}(x, t, \xi)\right| \mathrm{d} \xi \mathrm{d} x .
\end{aligned}
$$

Consequently [see (3.14), (3.15)],

$$
\begin{aligned}
& \lim _{\varepsilon, \delta \rightarrow 0} \int_{\left(\mathbb{T}^{N}\right)^{2}} \int_{\mathbb{R}^{2}} \rho_{\varepsilon}(x-y) \psi_{\delta}(\xi-\zeta) f_{1}^{ \pm}(x, t, \xi) \bar{f}_{2}^{ \pm}(y, t, \zeta) \mathrm{d} \xi \mathrm{d} \zeta \mathrm{d} x \mathrm{~d} y \\
& \quad=\int_{\mathbb{T}^{N}} \int_{\mathbb{R}} f_{1}^{ \pm}(x, t, \xi) \bar{f}_{2}^{ \pm}(x, t, \xi) \mathrm{d} \xi \mathrm{d} x
\end{aligned}
$$

for all $\omega \in \hat{\Omega}$. We apply the estimate (3.5). We have the uniform bounds

$$
\left\|\rho_{\varepsilon}\right\|_{L^{1}\left(\mathbb{T}^{N}\right)}=1, \quad\left\|\psi_{\delta}\right\|_{L^{1}(\mathbb{R})}=1, \quad R=\delta \leqq 1 .
$$

Consequently, we may apply the Lebesgue dominated convergence theorem to obtain

$$
\begin{aligned}
& \mathbb{E} \int_{\mathbb{T}^{N}} \int_{\mathbb{R}} f_{1}^{ \pm}(x, t, \xi) \bar{f}_{2}^{ \pm}(x, t, \xi) \mathrm{d} x \mathrm{~d} \xi \\
& \quad \leqq \mathbb{E} \int_{\left(\mathbb{T}^{N}\right)^{2}} \int_{\mathbb{R}^{2}} \rho_{\varepsilon}(x-y) \psi_{\delta}(\xi-\zeta) f_{1}^{ \pm}(x, t, \xi) \bar{f}_{2}^{ \pm}(y, t, \zeta) \mathrm{d} \xi \mathrm{d} \zeta \mathrm{d} x \mathrm{~d} y+\eta_{t}(\varepsilon, \delta),
\end{aligned}
$$

where $\lim _{\varepsilon, \delta \rightarrow 0} \eta_{t}(\varepsilon, \delta)=0$. Once (3.16) has been established, we may follow the lines of the proof of Theorem 11 in [14] to obtain (see Equation (31) in [14])

$$
\mathbb{E} \int_{\mathbb{T}^{N}} \int_{\mathbb{R}} f_{1}^{ \pm}(t) \bar{f}_{2}^{ \pm}(t) \mathrm{d} x \mathrm{~d} \xi \leqq \int_{\mathbb{T}^{N}} \int_{\mathbb{R}} f_{1,0} \bar{f}_{2,0} \mathrm{~d} x \mathrm{~d} \xi
$$

Assume that $f$ is a generalized solution to (1.1) with initial datum $\mathbf{1}_{u_{0}>\xi}$. Since $f_{0}$ is the (translated) Heavyside function $\mathbf{1}_{u_{0}>\xi}$, we have the identity $f_{0} \bar{f}_{0}=0$. Taking $f_{1}=f_{2}=f$ in (3.17), we deduce $f^{+}\left(1-f^{+}\right)=0$ almost everywhere, that is $f^{+} \in\{0,1\}$ almost everywhere. The fact that $-\partial_{\xi} f^{+}$is a Young measure then gives the conclusion; indeed, by Fubini's Theorem, for any $t \in[0, T]$, there is a set $E_{t}$ of full measure in $\mathbb{T}^{N} \times \Omega$ such that, for $(x, \omega) \in E_{t}, f^{+}(x, t, \xi, \omega) \in\{0,1\}$ for almost every $\xi \in \mathbb{R}$. Let

$$
\tilde{E}_{t}=E_{t} \cap\left(\mathbb{T}^{N} \times \hat{\Omega}\right) .
$$


The set $\tilde{E}_{t}$ is of full measure in $\mathbb{T}^{N} \times \Omega$. For $(x, \omega) \in \tilde{E}_{t},-\partial_{\xi} f^{+}(x, t, \cdot, \omega)$ is a probability measure on $\mathbb{R}$. Therefore $f^{+}(t, x, \xi, \omega)=\mathbf{1}_{u(x, t, \omega)>\xi}$ for almost every $\xi \in \mathbb{R}$, where $u(x, t, \omega)=\int_{\mathbb{R}}\left(f^{+}(x, t, \xi, \omega)-\mathbf{1}_{0>\xi}\right) \mathrm{d} \xi$. We have a similar result for $f^{-}$(this will be used in the proof of Corollary 3.3). Proposition 2.8 implies that $u$ is a solution in the sense of Definition 2.2. Since $f=f^{+}$(recall the convention of notation introduced at the beginning of Section 3.1), this shows the reduction of generalized solutions to solutions. If $u_{1}$ and $u_{2}$ are now two solutions to (1.1), we deduce from (3.17) with $f_{i}=\mathbf{1}_{u_{i}>\xi}$ and from the identity

$$
\int_{\mathbb{R}} \mathbf{1}_{u_{1}>\xi} \overline{\mathbf{1}_{u_{2}>\xi}} \mathrm{d} \xi=\left(u_{1}-u_{2}\right)^{+}
$$

the contraction property (3.8).

In the multiplicative case ( $\Phi$ depending on $u$ ), the reasoning is similar, see [14].

Proof of Corollary 3.3. We use the notations and the results of Proposition 2.10. We fix $p \in[1,+\infty)$. Both $(f(t))_{t \in[0, T]}$ and $\left(f^{-}(t)\right)_{t \in[0, T]}$ are generalized solutions to (1.1) associated with the initial datum $\mathbf{1}_{u_{0}>\xi}$ (we use (2.35) here). By Theorem 3.2, they are at equilibrium: $f(t)=\mathbf{1}_{u(t)>\xi}, f^{-}(t)=\mathbf{1}_{u^{-}(t)>\xi}$. By Proposition 2.13, for all $\omega \in \hat{\Omega}$, the map $t \mapsto u(t)$ from $[0, T]$ to $L^{p}\left(\mathbb{T}^{N}\right)$ is continuous from the right. Similarly, $t \mapsto u^{-}(t)$ is continuous from the left. By Proposition 2.11, the fact that $f^{-}$is at equilibrium has the following consequence: at every $t \in(0, T], f(t)=f^{-}(t)$. In particular, we have $u=u^{-}$and thus, almost surely, $u$ is continuous from $[0, T]$ to $L^{p}\left(\mathbb{T}^{N}\right)$.

We apply (3.8) to infer an $L^{\infty}$ bound on solutions to (1.1) in the particular case of a multiplicative noise with compact support.

Theorem 3.4. ( $L^{\infty}$ bounds). Assume (1.3)-(1.4) and

$$
g_{k}(x, u)=0, \quad \forall|u| \geqq 1,
$$

for all $x \in \mathbb{T}^{N}, k \geqq 1$. Let $u_{0} \in L^{\infty}\left(\mathbb{T}^{N}\right)$ satisfy $-1 \leqq u_{0} \leqq 1$ almost everywhere. Then, for all $t \geqq 0$, the solution $u$ to (1.1) with initial datum $u_{0}$ satisfies, almost surely,

$$
-1 \leqq u(x, t) \leqq 1
$$

almost everywhere in $\mathbb{T}^{N}$.

Proof of Theorem 3.4. We use (3.8) to compare $u$ to the two particular constant solutions $(x, t) \mapsto-1$ and $(x, t) \mapsto 1$.

Remark 3.3. (Comparative analysis between Sections 2 and 3 and Sections 2 and 3 of [14]). In Section 2, we introduce in Definition 2.2 a notion of solution that differs from the one given in [14, Definition 2]. The notions of generalized solution (Def. 2.6 here, Definition 7 in [14]) are different therefore. The elements on Young measure theory are common in both papers, though (compare for example Definition 2.3 with [14, Definition 3]). However this repetition seems necessary to obtain, 
to a certain extent, a self-consistent paper. Furthermore, the hypothesis "countably generated" on the $\sigma$-algebra $\mathcal{A}$ in [14, Theorem 5] was missing out (compare to the statement of Theorem 2.4). The analysis of left limits of solutions in Section 2.3, although similar, is different from the study in Section 2.3 in [14]. Indeed, right limits are known to exist in our present paper by essence (the càdlàg property is one of the criteria for being a (generalized) solution). More importantly, Proposition 2.10 is more specific than Proposition 8 of [14] about the almost certain character of the results. Note also that Proposition 2.11, its corollary 2.12, and Proposition 2.10, absent in [14], are necessary to prove the a.s. continuity of solutions (a point that was treated too quickly in [14, Proof of Corollary 12]). The method used here to prove uniqueness (and the reduction result for generalized solutions) is very similar to what was done in [14, Section 3], however, it is more complete [ $c f$. Remark 3.1, Formula (3.4) in particular, that is used to justify (3.11)]. The initial part of the proof of Proposition 3.1 differs from the proof of Proposition 9 in [14]. For the final arguments, we have referred to [14]. Eventually, although Theorem 3.2 and Corollary 3.3 are similar to Theorem 11 and Corollary 12 in [14], we have given their proofs with many details. It was required in the case of Corollary 3.3, as already mentioned. In the proof of Theorem 3.2, we explain with more precision than in [14] how to obtain (3.16). Then we essentially refer to the proof of [14, Theorem 11] since the arguments we used are the same.

\section{Convergence of Approximate Solutions}

In this section, we develop the tools required for the proof of convergence of a certain type of approximate solutions to (1.1). The basic principle is to generalize the notion of solution introduced in Definition 2.2. Indeed, this facilitates the proof of existence/convergence. In a second step a result of reduction (or "rigidity result"), which asserts that a generalized solution is a solution is used. This principle is of much use in the deterministic theory of conservation laws ( $c f$. [15] with the use of "measure-valued entropy solutions", [19] with the use of "entropy process solutions", [41] with the use of kinetic solutions as defined here). We have already introduced a generalization of the notion of solution in Definition 2.6, and have proved a result of reduction in Theorem 3.2. Here we will work mainly on the probabilistic aspects of the questions. We will have to consider "solutions in law", or "martingale solutions" (see the comment after Theorem 4.6 for more explanations about the terminology). The plan of this section is the following: in Section 4.1, we define the notion of approximate generalized solution. In Section 4.2, we give a martingale characterization of the stochastic integral. In Section 4.3, we give some tightness results on sequences of approximate generalized solutions. The main result, Theorem 4.6, which shows the convergence of a sequence of approximate generalized solutions to a martingale generalized solution, is proved in Section 4.5. Eventually, we obtain a result of pathwise convergence in Section 4.6.

\subsection{Approximate Generalized Solutions}

Let $\mathrm{d}$ be an integer fixed once and for all. 
Definition 4.1. (Approximate generalized solutions). Let $f_{0}^{n}: \mathbb{T}^{N} \times \mathbb{R} \rightarrow[0,1]$ be some kinetic functions. Let $\left(f^{n}(t)\right)_{t \in[0, T]}$ be a sequence of $L^{\infty}\left(\mathbb{T}^{N} \times \mathbb{R} ;[0,1]\right)$ valued processes. Assume that the functions $f^{n}(t)$, and the associated Young measures $v_{t}^{n}=-\partial_{\xi} \varphi f^{n}(t)$ are satisfying Items 1, 2, 3, in Definition 2.6 and Equation (2.23) up to an error term, that is: for all $\varphi \in C_{c}^{\mathrm{d}}\left(\mathbb{T}^{N} \times \mathbb{R}\right)$, there exists an adapted process $\varepsilon^{n}(t, \varphi)$, with $t \mapsto \varepsilon^{n}(t, \varphi)$ almost surely continuous such that

$$
\lim _{n \rightarrow+\infty} \sup _{t \in[0, T]}\left|\varepsilon^{n}(t, \varphi)\right|=0 \text { in probability, }
$$

and there exists some random measures $m^{n}$ with first moment (2.2), such that, for all $n$, for all $\varphi \in C_{c}^{\mathrm{d}}\left(\mathbb{T}^{N} \times \mathbb{R}\right)$, for all $t \in[0, T]$, almost surely,

$$
\begin{aligned}
\left\langle f^{n}(t), \varphi\right\rangle= & \left\langle f_{0}^{n}, \varphi\right\rangle+\int_{0}^{t}\left\langle f^{n}(s), a(\xi) \cdot \nabla_{x} \varphi\right\rangle \mathrm{d} s \\
& +\int_{0}^{t} \int_{\mathbb{T}^{N}} \int_{\mathbb{R}} g_{k}(x, \xi) \varphi(x, \xi) \mathrm{d} v_{x, s}^{n}(\xi) \mathrm{d} x \mathrm{~d} \beta_{k}(s)+\varepsilon^{n}(t, \varphi) \\
& +\frac{1}{2} \int_{0}^{t} \int_{\mathbb{T}^{N}} \int_{\mathbb{R}} \mathbf{G}^{2}(x, \xi) \partial_{\xi} \varphi(x, \xi) \mathrm{d} v_{x, s}^{n}(\xi) \mathrm{d} x \mathrm{~d} s-m^{n}\left(\partial_{\xi} \varphi\right)([0, t]) .
\end{aligned}
$$

Assume also $f^{n}(0)=f_{0}^{n}$. Then we say that $\left(f^{n}\right)$ is a sequence of approximate generalized solutions to (1.1) with initial datum $f_{0}^{n}$.

\subsection{Martingale Characterization of the Stochastic Integral}

In order to pass to the limit in an equation such as (4.2), we will first characterize (4.2) in terms of a martingale problem, and then we will use martingale methods to pass to the limit. In the present section, we give the characterization of (4.2) in terms of a martingale problem, see Proposition 4.1 and Proposition 4.2 below. We refer to [30, Example 1.4, p. 143] for characterization of the standard Wiener Process in terms of a martingale problem. In the context of SDEs and SPDEs, such kind of characterizations have been applied in $[7,13,26,29,40]$ in particular.

Let us define the stochastic integrands

$$
h_{\varphi, k}^{n}(t)=\int_{\mathbb{T}^{N}} \int_{\mathbb{R}} g_{k}(x, \xi) \varphi(x, \xi) \mathrm{d} \nu_{x, t}^{n}(\xi) \mathrm{d} x, \quad h_{\varphi}^{n}(t)=\left(h_{\varphi, k}^{n}(t)\right)_{k \geqq 1},
$$

and the stochastic integrals

$$
M_{\varphi}^{n}(t)=\sum_{k \geqq 1} \int_{0}^{t} \int_{\mathbb{T}^{N}} \int_{\mathbb{R}} g_{k}(x, \xi) \varphi(x, \xi) \mathrm{d} v_{x, s}^{n}(\xi) \mathrm{d} x \mathrm{~d} \beta_{k}(s)
$$

By Lemma 2.7, we have $h_{\varphi}^{n} \in L_{\mathcal{P}}^{2}\left([0, T] \times \Omega ; l^{2}\left(\mathbb{N}^{*}\right)\right)$ for all $n, \varphi$. Using Itō's Formula, we deduce from (4.4) the following statement: 
Proposition 4.1. Let $\left(f^{n}\right)$ be a sequence of approximate generalized solutions to (1.1) with initial datum $f_{0}^{n}$. Let $\varphi \in C_{c}^{\mathrm{d}}\left(\mathbb{T}^{N} \times \mathbb{R}\right)$. Let $M_{\varphi}^{n}(t)$ be defined by (4.4) and $h_{\varphi, k}^{n}(t)$ by (4.3). Then the processes

$$
M_{\varphi}^{n}(t), \quad M_{\varphi}^{n}(t) \beta_{k}(t)-\int_{0}^{t} h_{\varphi, k}^{n}(s) \mathrm{d} s, \quad\left|M_{\varphi}^{n}(t)\right|^{2}-\int_{0}^{t}\left\|h_{\varphi}^{n}(s)\right\|_{l^{2}\left(\mathbb{N}^{*}\right)} \mathrm{d} s,
$$

are $\left(\mathcal{F}_{t}\right)$-martingales.

What will interest us is the reciprocal statement.

Proposition 4.2. Let $h \in L_{\mathcal{P}}^{2}\left([0, T] \times \Omega ; l^{2}\left(\mathbb{N}^{*}\right)\right)$. Let $X(t)$ be a stochastic process starting from 0 such that the processes

$$
X(t), \quad X(t) \beta_{k}(t)-\int_{0}^{t} h_{k}(s) \mathrm{d} s, \quad|X(t)|^{2}-\int_{0}^{t}\|h(s)\|_{l^{2}\left(\mathbb{N}^{*}\right)}^{2} \mathrm{~d} s
$$

are $\left(\mathcal{F}_{t}\right)$-martingales. Then

$$
X(t)=\sum_{k \geqq 1} \int_{0}^{t} h_{k}(s) \mathrm{d} \beta_{k}(s)
$$

for all $t \in[0, T]$.

Proof of Proposition 4.2. The proof can be found in [26, Proposition A.1]. Let us give some details about it. We first claim that the following identity is satisfied:

$$
\mathbb{E}\left[(X(t)-X(s)) \int_{s}^{t} \theta(\sigma) \mathrm{d} \beta_{k}(\sigma) \mathrm{d} \sigma-\int_{s}^{t} h_{k}(\sigma) \theta(\sigma) \mathrm{d} \sigma \mid \mathcal{F}_{s}\right]=0
$$

for all $0 \leqq s \leqq t \leqq T$, all $k \geqq 1$ and all $\theta \in L_{\mathcal{P}}^{2}([0, T] \times \Omega)$. The proof consists in approximating $\theta$ on the interval $[s, t]$ by predictable simple functions. It is similar to a computation of quadratic variation. Note that (4.8) uses only the fact that

$$
X(t), \quad X(t) \beta_{k}(t)-\int_{0}^{t} h_{k}(s) \mathrm{d} s
$$

are $\left(\mathcal{F}_{t}\right)$-martingales. We apply (4.8) with $s=0$ and $\theta=h_{k}$ and sum over $k$ to obtain

$$
\mathbb{E}[X(t) \bar{X}(t)]=\mathbb{E} \int_{0}^{t}\|h(s)\|_{l^{2}\left(\mathbb{N}^{*}\right)}^{2} \mathrm{~d} s, \quad \bar{X}(t):=\sum_{k \geqq 1} \int_{0}^{t} h_{k}(s) \mathrm{d} \beta_{k}(s) .
$$

This gives the expression of the cross-product when we expand the term $\mathbb{E} \mid X(t)-$ $\left.\bar{X}(t)\right|^{2}$. Using the fact that

$$
|X(t)|^{2}-\int_{0}^{t}\|h(s)\|_{l^{2}\left(\mathbb{N}^{*}\right)}^{2} \mathrm{~d} s
$$


is a $\left(\mathcal{F}_{t}\right)$-martingale and applying Itō's Isometry to $\mathbb{E}|\bar{X}(t)|^{2}$ shows that the square terms are also given by

$$
\mathbb{E}|X(t)|^{2}=\mathbb{E}|\bar{X}(t)|^{2}=\int_{0}^{t}\|h(s)\|_{l^{2}\left(\mathbb{N}^{*}\right)}^{2} \mathrm{~d} s .
$$

It follows that $X(t)=\bar{X}(t)$.

\subsection{Tightness}

Let $\left(f^{n}\right)$ be a sequence of approximate generalized solutions, in the sense of Definition 4.1. Recall that $\mathcal{Y}^{1}$ is the notation for the set of Young measures on $\mathbb{T}^{N} \times[0, T] \times \mathbb{R}\left(c f\right.$. Proposition 2.3) and that $\mathcal{M}_{b}\left(\mathbb{T}^{N} \times[0, T] \times \mathbb{R}\right)$ is the notation for the set of bounded Borel measures on $\mathbb{T}^{N} \times[0, T] \times \mathbb{R}$ while $\mathcal{M}_{b}^{+}\left(\mathbb{T}^{N} \times[0, T] \times \mathbb{R}\right)$ is the subset of non-negative measures. Let $v^{n}$ be the Young measure associated to $f^{n}\left(v^{n}=-\partial_{\xi} f^{n}\right)$. The law of $v^{n}$ is a probability measure on the space $\mathcal{Y}^{1}$. We will see in Section 4.3.1 that, under a natural a priori bound, see (4.10), the sequence $\left(\operatorname{Law}\left(v^{n}\right)\right)$ is tight in $\mathcal{Y}^{1}$. In Section 4.3.2, this is the sequence $\left(\operatorname{Law}\left(m^{n}\right)\right)$ that we will analyse. We show under (4.14) and (4.15) that it is tight in $\mathcal{M}_{b}^{+}\left(\mathbb{T}^{N} \times[0, T] \times \mathbb{R}\right)$ (see, more specifically, Proposition 4.4).

We also need to analyse the tightness of $\left(\left\langle f_{n}(t), \varphi\right\rangle\right)$ in the Skorokhod space $D([0, T])$ : this is done in Section 4.3.3.

4.3.1. Compactness of the Young Measures In this section, we will use the following notions: we say that a sequence $\left(v^{n}\right)$ of $\mathcal{Y}^{1}$ converges to $v$ in $\mathcal{Y}^{1}$ if (2.15) is satisfied. A random Young measure is by definition a $\mathcal{Y}^{1}$-valued random variable.

Proposition 4.3. Let $\left(f^{n}\right)$ be a sequence of approximate generalized solutions to (1.1) with initial datum $f_{0}^{n}$. Assume that the following bound is satisfied: for all $p \in[1,+\infty)$, there exists $C_{p} \geqq 0$ independent on $n$ such that $v^{n}:=-\partial_{\xi} f^{n}$ satisfies

$$
\mathbb{E}\left[\sup _{t \in[0, T]} \int_{\mathbb{T}^{N}} \int_{\mathbb{R}}|\xi|^{p} \mathrm{~d} v_{x, t}^{n}(\xi) \mathrm{d} x\right] \leqq C_{p}
$$

Then, there exists a probability space $(\tilde{\Omega}, \tilde{\mathcal{F}}, \tilde{\mathbb{P}})$ and some random Young measures $\tilde{v}^{n}, \tilde{v}$, such that

1. $\tilde{v}^{n}$ has the same law as $v^{n}$,

2. $\tilde{v}$ satisfies

$$
\tilde{\mathbb{E}}\left(\sup _{J \subset[0, T]} \frac{1}{|J|} \int_{J} \int_{\mathbb{T}^{N}} \int_{\mathbb{R}}|\xi|^{p} \mathrm{~d} \tilde{\nu}_{x, t}(\xi) \mathrm{d} x \mathrm{~d} t\right) \leqq C_{p},
$$

where the supremum in (4.11) is a countable supremum over all open intervals $J \subset[0, T]$ with rational extremities, 
3. up to a subsequence still denoted $\left(\tilde{v}^{n}\right)$, there is $\tilde{\mathbb{P}}$-almost sure convergence of $\left(\tilde{v}^{n}\right)$ to $\tilde{v}$ in $\mathcal{Y}^{1}$.

Furthermore, if $\tilde{f}^{n}, \tilde{f}: \mathbb{T}^{N} \times[0, T] \times \mathbb{R} \times \tilde{\Omega} \rightarrow[0,1]$ are defined by

$$
\tilde{f}^{n}(x, t, \xi)=\tilde{v}_{x, t}^{n}(\xi,+\infty), \quad \tilde{f}(x, t, \xi)=\tilde{v}_{x, t}(\xi,+\infty),
$$

then $\tilde{f}^{n} \rightarrow \tilde{f}$ in $L^{\infty}\left(\mathbb{T}^{N} \times[0, T] \times \mathbb{R}\right)$-weak-* $\tilde{\mathbb{P}}$-almost surely, $\tilde{f}$ being a kinetic function.

Proof of Proposition 4.3. Note first that (4.10) yields

$$
\mathbb{E}\left(\int_{0}^{T} \int_{\mathbb{T}^{N}} \int_{\mathbb{R}}|\xi|^{p} \mathrm{~d} \nu_{x, t}^{n}(\xi) \mathrm{d} x \mathrm{~d} t\right) \leqq C_{p} T
$$

For $R>0, p \geqq 1$, let us denote by $K_{R, p}$ the set of Young measures $v \in \mathcal{Y}^{1}$ such that

$$
\int_{0}^{T} \int_{\mathbb{T}^{N}} \int_{\mathbb{R}}|\xi|^{p} \mathrm{~d} v_{x, t}(\xi) \mathrm{d} x \mathrm{~d} t \leqq R
$$

By [8, Theorems 4.3.2, 4.3.8, 2.1.3], the set $K_{R, p}$ is compact in $\mathcal{Y}^{1}$ for the $\tau_{\mathcal{Y}^{1}}^{W}$ topology, which is metrizable, [8, Theorem 2.3.1] and corresponds to the convergence (2.15). By (4.12), we have

$$
\mathbb{P}\left(v^{n} \notin K_{R, p}\right) \leqq \frac{C_{p} T}{R},
$$

which shows that the sequence $\left(v^{n}\right)$ of $\mathcal{Y}^{1}$-valued random variables is tight. The set $\mathcal{Y}^{1}$ endowed with the $\tau_{\mathcal{Y}^{1}}^{W}$-topology is Polish, [8, Theorem 2.3.3]: we can use the Prokhorov's metric, [6, p. 72]. By Prokhorov's Theorem, [6, Theorem 5.1], there exists a $\mathcal{Y}^{1}$-valued random variable $v$ and a subsequence still denoted $\left(v^{n}\right)$ such that $\left(v^{n}\right)$ converges in probability to $v$. Since the map

$$
\psi_{p}: \mathcal{Y}^{1} \rightarrow[0,+\infty], \quad v \mapsto \sup _{J \subset[0, T]} \frac{1}{|J|} \int_{J} \int_{\mathbb{T}^{N}} \int_{\mathbb{R}}|\xi|^{p} \mathrm{~d} v_{x, t}(\xi) \mathrm{d} x \mathrm{~d} t
$$

is lower semi-continuous, we have

$$
\mathbb{E} \psi_{p}(v) \leqq \liminf _{n \rightarrow+\infty} \mathbb{E} \psi_{p}\left(v^{n}\right) \leqq C_{p}
$$

by (4.10) and, consequently, that $v$ satisfies the condition

$$
\mathbb{E}\left(\sup _{J \subset[0, T]} \frac{1}{|J|} \int_{J} \int_{\mathbb{T}^{N}} \int_{\mathbb{R}}|\xi|^{p} \mathrm{~d} v_{x, t}(\xi) \mathrm{d} x \mathrm{~d} t\right) \leqq C_{p} .
$$

Let us now apply the Skorokhod Theorem [6, p. 70]. There exists a probability space $(\tilde{\Omega}, \tilde{\mathcal{F}}, \tilde{\mathbb{P}})$ and some random variables $\tilde{v}^{n}, \tilde{v}$, such that

1. $\tilde{v}^{n}$ and $\tilde{v}$ have the same laws as $v^{n}$ and $v$ respectively,

2. up to a subsequence still denoted $\left(\tilde{v}^{n}\right)$, there is $\tilde{\mathbb{P}}$-almost sure convergence of $\left(\tilde{v}^{n}\right)$ to $\tilde{v}$ in $\mathcal{Y}^{1}$.

Since $\tilde{v}$ and $v$ have same laws, $\tilde{v}$ satisfies the bound (4.11). If we apply Corollary 2.5, we obtain that $\tilde{f}^{n} \rightarrow \tilde{f}$ in $L^{\infty}\left(\mathbb{T}^{N} \times[0, T] \times \mathbb{R}\right)$-weak-* $\tilde{\mathbb{P}}$-almost surely, $\tilde{f}$ being a kinetic function. 


\subsubsection{Compactness of the Random Measures}

Proposition 4.4. Let $\left(f^{n}\right)$ be a sequence of approximate generalized solutions to (1.1) with initial datum $f_{0}^{n}$. Assume that

$$
\mathbb{E} m^{n}\left(\mathbb{T}^{N} \times[0, T] \times \mathbb{R}\right) \text { is uniformly bounded, }
$$

and that $m^{n}$ vanishes for large $\xi$ uniformly in $n:$ if $B_{R}^{c}=\{\xi \in \mathbb{R},|\xi| \geqq R\}$, then

$$
\lim _{R \rightarrow+\infty} \mathbb{E} m^{n}\left(\mathbb{T}^{N} \times[0, T] \times B_{R}^{c}\right)=0
$$

uniformly in $n$. Then, there exists a probability space $(\tilde{\Omega}, \tilde{\mathcal{F}}, \tilde{\mathbb{P}})$ and some random measures $\tilde{m}^{n}, \tilde{m}: \tilde{\Omega} \rightarrow \mathcal{M}_{b}\left(\mathbb{T}^{N} \times[0, T] \times \mathbb{R}\right)$ such that

1. $\tilde{m}^{n}$ has the same law as $m^{n}$,

2. up to a subsequence still denoted $\left(\tilde{m}^{n}\right)$, there is $\tilde{\mathbb{P}}$-almost sure convergence of $\left(\tilde{m}^{n}\right)$ to $\tilde{m}$ in $\mathcal{M}_{b}\left(\mathbb{T}^{N} \times[0, T] \times \mathbb{R}\right)$-weak-*.

Proof of Proposition 4.4. Let $\eta: \mathbb{R}_{+} \rightarrow \mathbb{R}_{+}$be defined by

$$
\eta(R)=\sup _{n \in \mathbb{N}} \mathbb{E} m^{n}\left(\mathbb{T}^{N} \times[0, T] \times B_{R}^{c}\right) .
$$

Let $h$ be a fixed function on $\mathbb{T}^{N} \times[0, T] \times \mathbb{R}, h$ continuous, positive, integrable. Proving the statement for the sequence of measures

$$
B \mapsto m^{n}(B)+\int_{B} h(x, t, \xi) \mathrm{d} x \mathrm{~d} t \mathrm{~d} \xi
$$

is equivalent to proving the statement for the original sequence $\left(m^{n}\right)$. We will assume therefore that $\eta(R)>0$ for all $R \geqq 0$ and that

$$
\left\|m^{n}\right\|:=m^{n}\left(\mathbb{T}^{N} \times[0, T] \times \mathbb{R}\right) \geqq \delta>0,
$$

where $\delta$ is independent on $n$. Let $\mu^{n}:=\frac{m^{n}}{\left\|m^{n}\right\|}$. We consider the random variables $X^{n}=\left(\mu^{n},\left\|m^{n}\right\|\right)$, taking values in $\mathcal{P}^{1}\left(\mathbb{T}^{N} \times[0, T] \times \mathbb{R}\right) \times \mathbb{R}_{+}$, where $\mathcal{P}^{1}\left(\mathbb{T}^{N} \times\right.$ $[0, T] \times \mathbb{R})$ is the set of probability measures on $\mathbb{T}^{N} \times[0, T] \times \mathbb{R}$. For $A>0$, let $K_{A}$ be the set of probability measures $\mu \in \mathcal{P}^{1}\left(\mathbb{T}^{N} \times[0, T] \times \mathbb{R}\right)$ such that

$$
\sup _{R>1} \frac{\mu\left(\mathbb{T}^{N} \times[0, T] \times B_{R}^{c}\right)}{\eta(R)} \leqq A .
$$

Then $K_{A}$ is compact in $\mathcal{P}^{1}\left(\mathbb{T}^{N} \times[0, T] \times \mathbb{R}\right)$-weak-* by Prokhorov's Theorem and (4.15). Using the Markov Inequality, and the definition of $\eta(R)$, we obtain

$$
\mathbb{P}\left(\mu^{n} \notin K_{A}\right) \leqq \frac{C}{A},
$$

where $C$ is independent on $n$; this shows that $\left(\mu^{n}\right)$ is tight in $\mathcal{P}^{1}\left(\mathbb{T}^{N} \times[0, T] \times\right.$ $\mathbb{R})$ endowed with the topology of the weak convergence of probability measures. Similarly, using (4.14) and the Markov Inequality, we have

$$
\mathbb{P}\left(\left\|m^{n}\right\|>A\right) \leqq \frac{C}{A},
$$


where $C$ is independent on $n$ :, therefore $\left(\left\|m^{n}\right\|\right)$ is tight in $\mathbb{R}$. It follows that $\left(X^{n}\right)$ is tight in $\mathcal{P}^{1}\left(\mathbb{T}^{N} \times[0, T] \times \mathbb{R}\right) \times \mathbb{R}_{+}$endowed with the product topology. This topology is separable, metrizable and there exists a compatible metric which turns the space into a complete space (we can take the sum of the Prokhorov's metric and of the usual metric on $\mathbb{R}_{+}$). Therefore we can apply the Skorokhod Theorem. There exists a probability space $(\tilde{\Omega}, \tilde{\mathcal{F}}, \tilde{\mathbb{P}})$ and some random variables $\tilde{X}^{n}=\left(\tilde{\mu}^{n}, \tilde{\alpha}^{n}\right)$, $\tilde{X}=(\tilde{\mu}, \tilde{\alpha})$ such that $\tilde{X}^{n}$ has same law as $X^{n}$ and, $\tilde{\mathbb{P}}$-almost surely, $\tilde{X}^{n} \rightarrow \tilde{X}$ in $\mathcal{P}^{1}\left(\mathbb{T}^{N} \times[0, T] \times \mathbb{R}\right) \times \mathbb{R}_{+}$. Set $\tilde{m}^{n}=\tilde{\alpha}^{n} \tilde{\mu}^{n}$ and $\tilde{m}=\tilde{\alpha} \tilde{\mu}$. Then $\tilde{m}^{n}$ has the same law as $m^{n}$ and there is $\tilde{\mathbb{P}}$-almost sure convergence of $\left(\tilde{m}^{n}\right)$ to $\tilde{m}$ in $\mathcal{M}_{b}\left(\mathbb{T}^{N} \times[0, T] \times \mathbb{R}\right)$ weak-*.

4.3.3. Tightness in the Skorokhod Space Let $D([0, T])$ denote the space of càdlàg functions on $[0, T]$. See $[30$, VI.1] and $[6$, Chapter 3$]$ for the definition of $D([0, T])$. Let $\left(f^{n}\right)$ be a sequence of approximate generalized solutions to (1.1) with initial datum $f_{0}^{n}$. In Section 4.4 below, where we analyse the convergence of $\left(f_{n}\right)$; it would be desirable to have a result of tightness of the processes $t \mapsto$ $\left\langle f^{n}(t), \varphi\right\rangle$ since they are random variables in $D([0, T])$ (here, $\varphi$ is a given testfunction). It seems difficult to obtain such a result. The only fact which we can infer naturally from (4.10), (4.14), (4.15), is that the sequence of processes

$$
t \mapsto\left\langle f^{n}(t), \varphi\right\rangle+A_{\varphi}^{n}(t), \quad A_{\varphi}^{n}(t):=\left\langle m^{n}, \partial_{\xi} \varphi\right\rangle([0, t])
$$

is tight in $D([0, T])$, see Proposition 4.5 below. Showing additionally that $\left(A_{\varphi}^{n}\right)$ is tight in $D([0, T])$ seems impossible, however, if no additional properties of $\left(\mathrm{m}^{n}\right)$ are known. Indeed, the weak convergence of $\mu^{n}:=\left\langle m^{n}, \partial_{\xi} \varphi\right\rangle$ to a measure $\mu$ on $[0, T]$ is not a sufficient condition for the convergence of $A_{\varphi}^{n}$ to $A(t)=\mu([0, t])$ in $D([0, T])$. Consider for example the case

$$
\mu^{n}=\delta_{t_{*}-s_{n}}+\delta_{t_{*}-\sigma_{n}},
$$

where $t_{*} \in(0, T)$ and $\left(s_{n}\right) \downarrow 0,\left(\sigma_{n}\right) \downarrow 0$ with $s_{n}<\sigma_{n}$ for all $n$. Then $\left(\mu_{n}\right)$ converges weakly to $\mu=2 \delta_{t_{*}}$, we have

$$
\alpha_{n}(t):=\mu_{n}([0, t]) \rightarrow \alpha(t):=\mu([0, t])
$$

for every $t \in[0, T]$, but $\left(\alpha_{n}\right)$, or any subsequence of $\left(\alpha_{n}\right)$, does not converge to $\alpha$ in $D([0, T])$. This example should be compared to [30, Example 1.20, p. 329]. See also Theorem 2.15, p. 342 in [30].

As asserted above, we will show that the sequence of processes

$$
t \mapsto\left\langle f^{n}(t), \varphi\right\rangle+A_{\varphi}^{n}(t), \quad A_{\varphi}^{n}(t):=\left\langle m^{n}, \partial_{\xi} \varphi\right\rangle([0, t]),
$$

where

$$
\left\langle m^{n}, \partial_{\xi} \varphi\right\rangle([0, t]):=\iiint_{\mathbb{T}^{N} \times[0, t] \times \mathbb{R}} \partial_{\xi} \varphi(x, s, \xi) \mathrm{d} m^{n}(x, s, \xi),
$$

is tight in $D([0, T])$. It is sufficient to show that

$$
t \mapsto\left\langle f^{n}(t), \varphi\right\rangle+B_{\varphi}^{n}(t), \quad B_{\varphi}^{n}(t):=\left\langle m^{n}, \partial_{\xi} \varphi\right\rangle([0, t])-\varepsilon^{n}(t, \varphi)
$$


is tight in $D([0, T])$ since each function $t \mapsto \varepsilon^{n}(t, \varphi)$ converges in probability to 0 in $C([0, T])$ by (4.1). Since $f^{n}(0)=f_{0}^{n}$, we have

$$
\left\langle f^{n}(t), \varphi\right\rangle+B_{\varphi}^{n}(t)=\left\langle f_{0}^{n}, \varphi\right\rangle+J_{\varphi}^{n}(t)
$$

$\mathbb{P}$-almost surely, where

$$
\begin{aligned}
& J_{\varphi}^{n}: t \mapsto \int_{0}^{t}\left\langle f^{n}(s), a(\xi) \cdot \nabla \varphi\right\rangle \mathrm{d} s \\
& +\sum_{k \geqq 1} \int_{0}^{t} \int_{\mathbb{T}^{N}} \int_{\mathbb{R}} g_{k}(x, \xi) \varphi(x, \xi) \mathrm{d} \nu_{x, s}^{n}(\xi) \mathrm{d} x \mathrm{~d} \beta_{k}(s) \\
& \quad+\frac{1}{2} \int_{0}^{t} \int_{\mathbb{T}^{N}} \int_{\mathbb{R}} \partial_{\xi} \varphi(x, \xi) \mathbf{G}^{2}(x, \xi) \mathrm{d} \nu_{x, s}^{n}(\xi) \mathrm{d} x \mathrm{~d} s .
\end{aligned}
$$

We will show that $\left(J_{\varphi}^{n}(t)\right)$ is tight in $C([0, T])$.

Proposition 4.5. Let $\left(f^{n}\right)$ be a sequence of approximate generalized solutions to (1.1) with initial datum $f_{0}^{n}$. For $\varphi \in C_{c}^{\mathrm{d}}\left(\mathbb{T}^{N} \times \mathbb{R}\right)$, set

$$
\begin{aligned}
D_{\varphi}^{n}(t) & =\int_{0}^{t}\left\langle f^{n}(s), a(\xi) \cdot \nabla \varphi\right\rangle \mathrm{d} s \\
M_{\varphi}^{n}(t) & =\sum_{k \geqq 1} \int_{0}^{t} \int_{\mathbb{T}^{N}} \int_{\mathbb{R}} g_{k}(x, \xi) \varphi(x, \xi) \mathrm{d} v_{x, s}^{n}(\xi) \mathrm{d} x \mathrm{~d} \beta_{k}(s), \\
I_{\varphi}^{n}(t) & =\frac{1}{2} \int_{0}^{t} \int_{\mathbb{T}^{N}} \int_{\mathbb{R}} \partial_{\xi} \varphi(x, \xi) \mathbf{G}^{2}(x, \xi) \mathrm{d} v_{x, s}^{n}(\xi) \mathrm{d} x \mathrm{~d} s .
\end{aligned}
$$

Assume that (4.10) is satisfied. Then each sequence $\left(D_{\varphi}^{n}\right),\left(M_{\varphi}^{n}\right),\left(I_{\varphi}^{n}\right)$ is tight in $C([0, T])$. In particular, the sequence $\left(J_{\varphi}^{n}\right)$ defined by (4.18) is tight in $C([0, T])$.

Proof of Proposition 4.5. Note first the trivial uniform bounds

$$
\mathbb{E}\left|D_{\varphi}^{n}(t)\right|, \mathbb{E}\left|M_{\varphi}^{n}(t)\right|, \mathbb{E}\left|I_{\varphi}^{n}(t)\right|=\mathcal{O}(1),
$$

which are obtained for $t=0$, since all three terms vanish. We then use Kolmogorov's criterion to obtain some bounds in some Hölder space $C^{\alpha}([0, T])$. We have the following estimate on the square of the increments of $D_{\varphi}^{n}$ :

$$
\mathbb{E}\left|D_{\varphi}^{n}(t)-D_{\varphi}^{n}(\sigma)\right|^{2} \leqq\|a \cdot \nabla \varphi\|_{L^{1}\left(\mathbb{T}^{N} \times \mathbb{R}\right)}^{2}|t-\sigma|^{2},
$$

since $\left|f_{n}\right| \leqq 1$ almost surely. Similarly, using (1.3) and (4.10), we have

$$
\mathbb{E}\left|I_{\varphi}^{n}(t)-I_{\varphi}^{n}(\sigma)\right|^{2} \leqq D_{0}^{2} T\left(1+C_{4}\right)\left\|\partial_{\xi} \varphi\right\|_{L^{\infty}\left(\mathbb{T}^{N} \times \mathbb{R}\right)}^{2}|t-\sigma|^{2} .
$$

The estimates (4.19) and (4.20) give some bounds on $\mathbb{E}\left\|D_{\varphi}^{n}\right\|_{C^{\alpha}([0, T])}$ and $\mathbb{E}\left\|I_{\varphi}^{n}\right\|_{C^{\alpha}([0, T])}$ respectively, for $\alpha<\frac{1}{2}$. Furthermore, the Burkholder - Davis - 
Gundy Inequality gives, for $p>2$,

$$
\begin{aligned}
& \mathbb{E}\left|M_{\varphi}^{n}(t)-M_{\varphi}^{n}(\sigma)\right|^{p} \\
& \quad \leqq \mathbb{E}\left[\sup _{\sigma \leqq r \leqq t}\left|M_{\varphi}^{n}(r)-M_{\varphi}^{n}(\sigma)\right|\right]^{p} \\
& \quad \leqq C_{\mathrm{BDG}}(p) \mathbb{E}\left[\sum_{k \geqq 1} \int_{\sigma}^{t}\left|\int_{\mathbb{T}^{N}} \int_{\mathbb{R}} g_{k}(x, \xi) \varphi(x, \xi) \mathrm{d} \nu_{x, s}^{n}(\xi) \mathrm{d} x\right|^{2} \mathrm{~d} s\right]^{p / 2} .
\end{aligned}
$$

By Jensen's Inequality, and a bound analogous to (2.25), we obtain

$$
\mathbb{E}\left|M_{\varphi}^{n}(t)-M_{\varphi}^{n}(\sigma)\right|^{p} \leqq C_{\mathrm{BDG}}(p)\left[D_{0}\left(1+C_{2}\right)\right]^{p / 2}\|\varphi\|_{L^{\infty}\left(\mathbb{T}^{N} \times \mathbb{R}\right)}^{p}|t-\sigma|^{p / 2},
$$

and (4.21) gives a bound on $\mathbb{E}\left\|M_{\varphi}^{n}\right\|_{C^{\alpha}([0, T])}$ for $\alpha<\frac{1}{2}-\frac{1}{p}$. We obtain in this way some tightness conditions on the laws of $D^{n}, M^{n}, I^{n}$ respectively on $C([0, T])$.

\subsection{Convergence of Approximate Generalized Solutions}

We conclude here this section about the stability of generalized solutions by the following statement:

Theorem 4.6. (Convergence to martingale solutions). Let $\left(f^{n}\right)$ be a sequence of approximate generalized solutions to (1.1) with initial datum $f_{0}^{n}$, satisfying (4.10), (4.14) and (4.15). We suppose that there exists a kinetic function $f_{0}$ on $\mathbb{T}^{N} \times$ $\mathbb{R}$ such that $f_{0}^{n} \rightarrow f_{0}$ in $L^{\infty}\left(\mathbb{T}^{N} \times \mathbb{R}\right)$-weak-*. Then there exists a probability space $(\tilde{\Omega}, \tilde{\mathcal{F}}, \tilde{\mathbb{P}})$, a filtration $\tilde{\mathcal{F}}_{t}$, some $\tilde{\mathcal{F}}_{t}$-adapted independent Brownian motions $\left(\tilde{\beta}_{k}\right)_{k \geqq 1}$, some random Young measures $\tilde{v}^{n}$, $\tilde{v}$, some random measures $\tilde{m}^{n}, \tilde{m}$ on $\mathbb{T}^{N} \times[0, T] \times \mathbb{R}$ such that

1. $\tilde{v}^{n}$ has the same law as $v^{n}$,

2. up to a subsequence still denoted $\left(\tilde{v}^{n}\right)$, there is $\tilde{\mathbb{P}}$-almost sure convergence of $\left(\tilde{v}^{n}\right)$ to $\tilde{v}$ in $\mathcal{Y}^{1}$,

3. for all $\psi \in C_{b}(\mathbb{R})$, the random map $(x, t) \mapsto\left\langle\psi, \tilde{v}_{x, t}\right\rangle$ belongs to $L_{\tilde{\mathcal{P}}}^{2}\left(\mathbb{T}^{N} \times\right.$ $[0, T] \times \tilde{\Omega})$,

4. $\tilde{m}^{n}$ has the same law as $m^{n}$,

5. up to a subsequence still denoted $\left(\tilde{m}^{n}\right)$, there is $\tilde{\mathbb{P}}$-almost sure convergence of $\left(\tilde{m}^{n}\right)$ to $\tilde{m}$ in $\mathcal{M}_{b}\left(\mathbb{T}^{N} \times[0, T] \times \mathbb{R}\right)$-weak-*.

Let $\tilde{f}$ be defined by $\tilde{f}(x, t, \xi)=\tilde{v}_{x, t}(\xi,+\infty)$, then, $\tilde{\mathbb{P}}$-almost surely, $\tilde{f}$ is a kinetic function and

6. up to a subsequence, and $\tilde{\mathbb{P}}$-almost surely, $\tilde{f}^{n}$ converges in $L^{\infty}\left(\mathbb{T}^{N} \times[0, T] \times\right.$ $\mathbb{R})$-weak-* to $\tilde{f}$,

7. $\tilde{\mathbb{P}}$-almost surely, for all $\varphi$ in $C_{c}\left(\mathbb{T}^{N} \times \mathbb{R}\right), t \mapsto\langle\tilde{f}(t), \varphi\rangle$ is càdlàg, 
8. $\tilde{v}$ satisfies

$$
\tilde{\mathbb{E}}\left(\sup _{t \in[0, T]} \int_{\mathbb{T}^{N}} \int_{\mathbb{R}}|\xi|^{p} \mathrm{~d} \tilde{\nu}_{x, t}(\xi) \mathrm{d} x\right) \leqq C_{p},
$$

for all $1 \leqq p<+\infty$, where $C_{p}$ is a finite constant,

9. for all $\varphi \in C_{c}^{1}\left(\mathbb{T}^{N} \times \mathbb{R}\right)$, for all $t \in[0, T], \tilde{\mathbb{P}}$-almost surely, $\tilde{f}$ satisfies

$$
\begin{aligned}
\langle\tilde{f}(t), \varphi\rangle= & \left\langle f_{0}, \varphi\right\rangle+\int_{0}^{t}\left\langle\tilde{f}(s), a(\xi) \cdot \nabla_{x} \varphi\right\rangle \mathrm{d} s \\
& +\int_{0}^{t} \int_{\mathbb{T}^{N}} \int_{\mathbb{R}} g_{k}(x, \xi) \varphi(x, \xi) \mathrm{d} \tilde{\nu}_{x, s}(\xi) \mathrm{d} x d \tilde{\beta}_{k}(s) \\
& +\frac{1}{2} \int_{0}^{t} \int_{\mathbb{T}^{N}} \int_{\mathbb{R}} \mathbf{G}^{2}(x, \xi) \partial_{\xi} \varphi(x, \xi) \mathrm{d} \tilde{\nu}_{x, s}(\xi) \mathrm{d} x \mathrm{~d} s-\tilde{m}\left(\partial_{\xi} \varphi\right)([0, t]) .
\end{aligned}
$$

After one does the substitution

$$
\left(\Omega, \mathcal{F}, \mathbb{P}, \mathcal{F}_{t}, \beta_{k}(t)\right) \leftarrow\left(\tilde{\Omega}, \tilde{\mathcal{F}}, \tilde{\mathbb{P}}, \tilde{\mathcal{F}}_{t}, \tilde{\beta}_{k}(t)\right),
$$

which is a substitution of the probabilistic data in the Cauchy Problem for Equation (1.1), the points $3,7,8,9$ in Theorem 4.6 show that $\tilde{f}$ is a generalized solution associated with the initial datum $f_{0}$. Such a function $\tilde{f}$, which turns out to be a generalized solution to (1.1) after a substitution of the probabilistic data, is called a martingale generalized solution. The term martingale refers to the martingale characterization of (4.23), cf. Proposition 4.1 and Proposition 4.2, which we will use to prove Theorem 4.6.

\subsection{Proof of Theorem 4.6}

In this section, we will give the proof of Theorem 4.6. We will use the results (and the proofs) of Proposition 4.3, Proposition 4.4, see Sections 4.3.1 and 4.3.2, respectively.

4.5.1. State Space and Skorokhod's Theorem Recall that

$$
W(t)=\sum_{k \geqq 1} \beta_{k}(t) e_{k},
$$

where $\left(e_{k}\right)_{k \geq 1}$ is the orthonormal basis of the Hilbert space $H$. Let $\mathfrak{U}$ be an other separable Hilbert space such that $H \hookrightarrow \mathfrak{U}$ with Hilbert-Schmidt injection. Then the trajectories of $W$ are $\mathbb{P}$-a.s. in the path-space $\mathcal{X}_{W}=C([0, T] ; \mathfrak{U}$ ) (see $[12$, Theorem 4.3]). We consider the $C^{\mathrm{d}}$-norm

$$
\|\varphi\|_{C^{\mathrm{d}}}=\sup \left\{\left\|D^{m} \varphi\right\|_{L^{\infty}\left(\mathbb{T}^{N} \times \mathbb{R}\right)} ; m \in\{0, \ldots, \mathrm{d}\}^{N+1}\right\}
$$

on $C_{c}^{\mathrm{d}}\left(\mathbb{T}^{N} \times \mathbb{R}\right)$. Let

$$
\Gamma=\left\{\varphi_{1}, \varphi_{2}, \ldots\right\}
$$


be a dense countable subset of $C_{c}^{\mathrm{d}}\left(\mathbb{T}^{N} \times \mathbb{R}\right)$ for this norm. We can construct $\Gamma$ as follows: let

$$
\rho_{\varepsilon}(x, \xi):=\frac{1}{\varepsilon^{N+1}} \rho\left(\varepsilon^{-N} x, \varepsilon^{-1} \xi\right)
$$

be a compactly supported approximation of the unit on $\mathbb{T}^{N} \times \mathbb{R}$. Let $\left\{\theta_{p} ; p \in \mathbb{N}\right\}$ be a dense subset of $L^{1}\left(\mathbb{T}^{N} \times \mathbb{R}\right)$. We can assume that all the functions $\theta_{p}$ are compactly supported (otherwise, we use a process of truncation). Then any function in $C_{c}^{\mathrm{d}}\left(\mathbb{T}^{N} \times \mathbb{R}\right)$ can be approximated by functions in

$$
\Gamma:=\left\{\rho_{k^{-1}} * \theta_{p} ; p \in \mathbb{N}, k \in \mathbb{N}^{*}\right\} \subset C_{c}^{\mathrm{d}}\left(\mathbb{T}^{N} \times \mathbb{R}\right)
$$

for the convergence measured by the $C^{\mathrm{d}}$-norm. Indeed, given $\varphi \in C_{c}^{\mathrm{d}}\left(\mathbb{T}^{N} \times \mathbb{R}\right)$, $a>0$, and $m \in\{0, \ldots, \mathrm{d}\}^{N+1}$, we have, by the triangular inequality,

$$
\begin{aligned}
\left\|D^{m} \varphi-D^{m} \rho_{\varepsilon} * \theta_{p}\right\|_{L^{\infty}} & \leqq\left\|D^{m} \varphi-D^{m} \rho_{\varepsilon} * \varphi\right\|_{L^{\infty}}+\left\|D^{m} \rho_{\varepsilon} *\left(\varphi-\theta_{p}\right)\right\|_{L^{\infty}} \\
& \leqq \omega_{D^{m} \varphi}(\varepsilon)+\frac{\|\rho\|_{L^{\infty}}}{\varepsilon^{N+1+|m|}}\left\|\varphi-\theta_{p}\right\|_{L^{1}}
\end{aligned}
$$

since the norm of $D^{m} \rho_{\varepsilon}$ in $L^{\infty}$ is bounded by $\frac{\|\rho\|_{L^{\infty}}}{\varepsilon^{N+1+|m|}}$. In (4.24), $\omega_{D^{m}} \varphi$ denotes the modulus of continuity of $D^{m} \varphi$. We choose $\varepsilon=k^{-1}$ with $k$ large enough to ensure $\omega_{D^{m}} \varphi(\varepsilon)<a$ for all $m \in\{0, \ldots, \mathrm{d}\}^{N+1}$. Then taking $p \in \mathbb{N}$ such that $\left\|\varphi-\theta_{p}\right\|_{L^{1}}<a \varepsilon^{(\mathrm{d}+1)(N+1)}$, we obtain $\left\|\varphi-\rho_{k^{-1}} * \theta_{p}\right\|_{C^{\mathrm{d}}}<2 a$.

Also, let $\mathbb{R}^{\infty}$ denote the product space $\prod_{\varphi \in \Gamma} \mathbb{R}$ endowed with the topology of point-wise convergence. As such, $\mathbb{R}^{\infty}$ is separable, complete and admits a compatible metric. Define the Polish space

$$
\mathcal{E}:=C\left([0, T] ; \mathbb{R}^{\infty}\right) \times C\left([0, T] ; \mathbb{R}^{\infty}\right) \times C\left([0, T] ; \mathbb{R}^{\infty}\right) \times \mathbb{R}^{\infty},
$$

and

$$
\varepsilon_{\varphi}^{n}(t)=\varepsilon^{n}(t, \varphi), \quad J_{\varphi}^{n}(t)=\left\langle f^{n}(t), \varphi\right\rangle-\left\langle f_{0}^{n}, \varphi\right\rangle+\left\langle m^{n}, \partial_{\xi} \varphi\right\rangle([0, t])-\varepsilon_{\varphi}^{n}(t),
$$

for all $\varphi \in C_{c}^{\mathrm{d}}\left(\mathbb{T}^{N} \times \mathbb{R}\right)$. Note that, as a consequence of Equation (4.17) and Proposition 4.5 , we know that, for all $\varphi \in C_{c}^{\mathrm{d}}\left(\mathbb{T}^{N} \times \mathbb{R}\right)$,

$$
\left(J_{\varphi}^{n}\right) \text { is tight in } C([0, T]) \text {. }
$$

By (4.1), we also have $\varepsilon_{\varphi}^{n} \rightarrow 0$ in $C([0, T])$ in probability, for all $\varphi \in C_{c}^{\mathrm{d}}\left(\mathbb{T}^{N} \times \mathbb{R}\right)$. We introduce the four following sequences:

$$
\left\{J^{n}(t)\right\}:=\left(J_{\varphi}^{n}(t)\right)_{\varphi \in \Gamma}, \quad\left\{M^{n}(t)\right\}:=\left(M_{\varphi}^{n}(t)\right)_{\varphi \in \Gamma}, \quad\left\{\varepsilon^{n}(t)\right\}:=\left(\varepsilon_{\varphi}^{n}(t)\right)_{\varphi \in \Gamma},
$$

and $\left\{f_{\text {in }}^{n}\right\}:=\left(\left\langle f_{0}^{n}, \varphi\right\rangle\right)_{\varphi \in \Gamma}$, where $M_{\varphi}^{n}$ is defined by (4.4). We will consider the multiplet

$$
Z^{n}=\left(v^{n},\left\{J^{n}\right\},\left\{M^{n}\right\},\left\{\varepsilon^{n}\right\},\left\{f_{\text {in }}^{n}\right\}, \mu^{n},\left\|m^{n}\right\|, W\right) \in \mathcal{X},
$$


where the state space $\mathcal{X}$ is

$$
\mathcal{X}:=\mathcal{Y}^{1} \times \mathcal{E} \times \mathcal{P}^{1}\left(\mathbb{T}^{N} \times[0, T] \times \mathbb{R}\right) \times \mathbb{R}_{+} \times \mathcal{X}_{W} .
$$

Let $\varepsilon>0$. By (4.26), there exists for each $j \in \mathbb{N}$ a compact $K_{j}$ in $C([0, T])$ such that

$$
\inf _{n \in \mathbb{N}} \mathbb{P}\left(J_{\varphi_{j}}^{n} \in K_{j}\right) \geqq 1-\frac{\varepsilon}{2^{j}} .
$$

Let $K=\prod_{j \in \mathbb{N}} K_{j}$. Then $K$ is compact in $C\left([0, T] ; \mathbb{R}^{\infty}\right)$ and

$$
\mathbb{P}\left(\left\{J^{n}\right\} \in K^{c}\right) \leqq \sum_{j \in \mathbb{N}} \mathbb{P}\left(J_{\varphi_{j}}^{n} \in K_{j}^{c}\right) \leqq \sum_{j \in \mathbb{N}} \frac{\varepsilon}{2^{j}}=2 \varepsilon
$$

for all $n \in \mathbb{N}^{2}$ This shows that $\left(\left\{J^{n}\right\}\right)$ is tight in $C\left([0, T] ; \mathbb{R}^{\infty}\right)$. We have similar results about $\left(\left\{M^{n}\right\}\right)$ and $\left(\left\{\varepsilon^{n}\right\}\right)$ by Proposition 4.5. On $\mathcal{X}_{W}$ we consider the topology induced by the norm

$$
\|v\|=\sup _{t \in[0, T]}\|v(t)\|_{\mathfrak{U}} .
$$

Then $\mathcal{X}_{W}$ is separable and complete. A first consequence of this is the fact that the law of the single random variable $W$ is tight in $\mathcal{X}_{W}$. A second consequence is the fact that $\mathcal{X}$ is a separable completely-metrizable space. By Sections 4.3.1 and 4.3.2, we conclude that $\left(Z^{n}\right)$ is tight in the Polish space $\mathcal{X}$. We may thus apply Skorokhod's Theorem to $\left(Z^{n}\right)$ : there exists a probability space $(\tilde{\Omega}, \tilde{\mathcal{F}}, \tilde{\mathbb{P}})$ and some random variable $\tilde{Z}^{n}, \tilde{Z}$ such that $\tilde{Z}^{n}$ has the same law as $Z^{n}$ and, up to a subsequence, $\tilde{\mathbb{P}}$-almost surely, $\tilde{Z}^{n}$ converges to $\tilde{Z}$ in $\mathcal{X}$.

4.5.2. Identification of the Limit: Càdlàg version Let us denote the component of $\tilde{Z}$ as follows:

$$
\tilde{Z}=\left(\tilde{v},\{\tilde{J}\},\{\tilde{M}\},\{\tilde{\varepsilon}\},\left\{\tilde{f}_{\text {in }}\right\}, \tilde{\mu}, \tilde{\alpha}, \tilde{W}\right)
$$

Note first that $\tilde{\varepsilon}=0$ by (4.1). We also have

$$
\left\{\tilde{f}_{\text {in }}\right\}=\left(\left\langle f_{0}, \varphi\right\rangle\right)_{\varphi \in \Gamma},
$$

since $f_{0}^{n} \rightarrow f_{0}$ in $L^{\infty}\left(\mathbb{T}^{N} \times \mathbb{R}\right)$-weak-* by hypothesis. Recall (see Proposition 4.3 and Proposition 4.4) that $\tilde{f}(x, t, \xi)=\tilde{v}_{x, t}(\xi,+\infty)$ and $\tilde{m}=\tilde{\alpha} \tilde{\mu}$. It was shown in the proof of Proposition 4.3 that item 1, 2, 6 of Theorem 4.6 are satisfied and that the moments of $\tilde{v}$ are bounded as in (4.11). By the proof of Proposition 4.4, we have also 4, 5 of Theorem 4.6. We will first establish the following result:

\footnotetext{
${ }^{2} \mathrm{~K}$ is compact since $C\left([0, T] ; \mathbb{R}^{\infty}\right)$ is homeomorphic to the countable product, over $\Gamma$, of copies of $C([0, T] ; \mathbb{R})$.
} 
Lemma 4.7. We have the following identities: $\tilde{\mathbb{P}}$-almost surely

$$
\begin{aligned}
\text { for all } t \in[0, T], \text { for all } \varphi \in \Gamma,\left\langle\tilde{f}^{n}(t), \varphi\right\rangle= & \left\langle f_{0}, \varphi\right\rangle+\tilde{J}_{\varphi}^{n}(t) \\
& -\left\langle\tilde{m}^{n}, \partial \xi \varphi([0, t])-\tilde{\varepsilon}_{\varphi}^{n}(t),\right.
\end{aligned}
$$

and for $\tilde{\mathbb{P}}$-almost surely there exists a negligible set $N_{0} \subset[0, T]$ such that

$$
\begin{aligned}
& \text { for all } t \in[0, T] \backslash N_{0}, \text { for all } \varphi \in \Gamma,\langle\tilde{f}(t), \varphi\rangle=\left\langle f_{0}, \varphi\right\rangle+\tilde{J}_{\varphi}(t) \\
& -\left\langle\tilde{m}, \partial_{\xi} \varphi\right\rangle([0, t]) \text {. }
\end{aligned}
$$

We will use (4.28) to prove Proposition 4.8, where we obtain a càdlàg version of $\tilde{f}$ (càdlàg in the sense that $\tilde{\mathbb{P}}$-almost surely, for all $\varphi \in C_{c}\left(\mathbb{T}^{N} \times \mathbb{R}\right), t \mapsto\langle\tilde{f}(t), \varphi\rangle$ is càdlàg).

Proof of Lemma 4.7. Let $\theta \in C([0, T])$. Let us integrate the identity (4.25) against $\theta$. Using the Fubini theorem, we obtain: $\mathbb{P}$-almost surely,

$$
\int_{0}^{T}\left(J_{\varphi}^{n}(t)+\left\langle f_{0}^{n}, \varphi\right\rangle+\varepsilon_{\varphi}^{n}(t)\right) \theta(t) \mathrm{d} t-\left\langle v^{n}, \Phi\right\rangle-\left\langle m^{n}, \Psi\right\rangle=0,
$$

where

$$
\Psi(x, t, \xi)=\partial_{\xi} \varphi(x, \xi) \int_{t}^{T} \theta(s) \mathrm{d} s, \quad \Phi(x, t, \xi)=\int_{-\infty}^{\xi} \varphi(x, \zeta) \mathrm{d} \zeta \theta(t) .
$$

Note that $\Psi$ and $\Phi$ are continuous and bounded functions. Taking the square, then expectancy in (4.29) gives $\mathbb{E} F\left(Z^{n}\right)=0$, where $F: \mathcal{X} \rightarrow \mathbb{R}$ defined by

$$
F\left(Z^{n}\right)=\left|\int_{0}^{T}\left(J_{\varphi}^{n}(t)+\left\langle f_{0}^{n}, \varphi\right\rangle+\varepsilon_{\varphi}^{n}(t)\right) \theta(t) \mathrm{d} t-\left\langle v^{n}, \Phi\right\rangle-\left\|m^{n}\right\|\left\langle\mu^{n}, \Psi\right\rangle\right|^{2}
$$

is a continuous function. By identity of the laws of $Z^{n}$ and $\tilde{Z}^{n}$, we have $\tilde{\mathbb{E}} F\left(\tilde{Z}^{n}\right)=0$ for all $n$. Since $F$ is non-negative, this means $F\left(\tilde{Z}^{n}\right)=0, \tilde{\mathbb{P}}$-almost surely. Since $\theta$ is arbitrary and $\Gamma$ is countable, we deduce (4.27), a priori for $t \in[0, T] \backslash N_{n}$, where $N_{n}$ is a measurable negligible set. We can take $N_{n}=\emptyset$ because both sides of (4.27) are càdlàg functions. By almost sure convergence, that $F\left(\tilde{Z}^{n}\right)=0, \tilde{\mathbb{P}}$-almost surely implies $F(\tilde{Z})=0, \tilde{\mathbb{P}}$-almost surely. Hence, similarly, we obtain (4.28).

Proposition 4.8. There exists a measurable subset $\tilde{\Omega}^{+}$of $\tilde{\Omega}$ of probability one, a random Young measure $\tilde{v}^{+}$on $\mathbb{T}^{N} \times(0, T)$ such that

1. for all $\tilde{\omega} \in \tilde{\Omega}^{+}$, for almost every $(x, t) \in \mathbb{T}^{N} \times(0, T)$, the probability measures $\tilde{v}_{x, t}^{+}$and $\tilde{v}_{x, t}$ coincide,

2. the kinetic function $\tilde{f}^{+}(x, t, \xi):=\tilde{v}_{x, t}^{+}(\xi,+\infty)$ satisfies: for all $\tilde{\omega} \in \tilde{\Omega}^{+}$, for all $\varphi \in C_{c}\left(\mathbb{T}^{N} \times(0, T)\right), t \mapsto\left\langle\tilde{f}^{+}(t), \varphi\right\rangle$ is càdlàg,

3. the random Young measure $\tilde{v}^{+}$satisfies (4.22). 
Proof of Proposition 4.8. The proof is quite similar to the proof of Proposition 2.10. For $\varphi \in \Gamma$, let $F_{\varphi}(t)$ denote the right-hand side of (4.28):

$$
F_{\varphi}(t)=\left\langle f_{0}, \varphi\right\rangle+\tilde{J}_{\varphi}(t)-\left\langle\tilde{m}, \partial_{\xi} \varphi\right\rangle([0, t]) .
$$

We define $\tilde{\Omega}^{+}$as the intersection of the three following events: first (4.28), second: "for all $\varphi \in \Gamma, F_{\varphi}$ is càdlàg", third the event

$$
\sup _{J \subset[0, T]} \frac{1}{|J|} \int_{J} \int_{\mathbb{T}^{N}} \int_{\mathbb{R}}|\xi|^{p} \mathrm{~d} \tilde{\nu}_{x, t}(\xi) \mathrm{d} x \mathrm{~d} t<+\infty,
$$

where the supremum over intervals $J$ is as in (4.11) (a countable supremum over all open intervals $J \subset[0, T]$ with rational extremities). Assume that $\tilde{\Omega}^{+}$is realized (say we draw a particular $\tilde{\omega} \in \tilde{\Omega}^{+}$). Assume in particular that

$$
\frac{1}{|J|} \int_{J} \int_{\mathbb{T}^{N}} \int_{\mathbb{R}}|\xi|^{p} \mathrm{~d} \tilde{\nu}_{x, t}(\xi) \mathrm{d} x \mathrm{~d} t \leqq C_{p}(\tilde{\omega})
$$

for all open intervals $J \subset[0, T]$ with rational extremities. Then the map

$$
t \mapsto \int_{\mathbb{T}^{N}} \int_{\mathbb{R}}|\xi|^{p} \mathrm{~d} \tilde{v}_{x, t}(\xi) \mathrm{d} x
$$

is integrable on $(0, T)$. A simple approximation procedure shows then that (4.31) holds true when $J$ is any interval in $[0, T]$.

Let $t_{*} \in[0, T)$. Let $\left(\varepsilon_{l}\right)$ be a sequence of positive numbers decreasing to 0 such that $t_{*}+\varepsilon_{1}<T$. Let $J_{l}=\left(t_{*}, t_{*}+\varepsilon_{l}\right)$. Consider the sequence of Young measures, and corresponding kinetic functions

$$
\tilde{v}_{x}^{(l)}=\frac{1}{\left|J_{l}\right|} \int_{J_{l}} \tilde{v}_{x, t} \mathrm{~d} t, \quad \tilde{f}^{(l)}(x, \xi)=\tilde{v}_{x}^{(l)}(\xi,+\infty)=\frac{1}{\left|J_{l}\right|} \int_{J_{l}} \tilde{f}(x, t, \xi) \mathrm{d} t .
$$

Since the Borel $\sigma$-algebra of $\mathbb{T}^{N}$ is countably generated ( $\mathbb{T}^{N}$ being separable), we can apply Theorem 2.4 and Corollary 2.5. There exists a subsequence $\left(l_{m}\right)$ and a Young measure $\tilde{v}^{*}$ such that $\tilde{v}^{\left(l_{m}\right)} \rightarrow \tilde{v}^{*}$ in the sense of $(2.15)$ and $\tilde{f}^{\left(l_{m}\right)} \rightarrow \tilde{f}^{*}$ in $L^{\infty}\left(\mathbb{T}^{N} \times \mathbb{R}\right)$ weak-*, where $\tilde{f}^{*}(x, \xi)=\tilde{v}_{x}^{*}(\xi,+\infty)$. The limit $\tilde{f}^{*}$ is unique. Indeed, if $\varphi \in \Gamma$, then, due to (4.28) and to the Fubini theorem, and due to the right-continuity of $F_{\varphi}$, we have

$$
\left\langle\tilde{f}^{(l)}, \varphi\right\rangle=\frac{1}{\left|J_{l}\right|} \int_{J_{l}} F_{\varphi}(t) \mathrm{d} t \rightarrow F_{\varphi}\left(t_{*}\right) .
$$

This implies

$$
\left\langle\tilde{f}^{*}, \varphi\right\rangle=F_{\varphi}\left(t_{*}\right)
$$

Since $\Gamma$ is dense, $\tilde{f}^{*}$ and $\tilde{\nu}^{*}=-\partial \xi \tilde{f}^{*}$ are unique. We deduce that the convergence holds along the whole sequence $l=1,2 \ldots$, independently on $\tilde{\omega} \in \tilde{\Omega}^{+}$and on $t_{*} \in[0, T)$. Consequently, setting

$$
\tilde{v}_{x, t_{*}}^{+}=\tilde{v}_{x}^{*}, \quad \tilde{f}^{+}\left(x, t_{*}, \xi\right)=\tilde{v}_{x, t_{*}}^{+}(\xi,+\infty),
$$


we have, for all $\tilde{\omega} \in \tilde{\Omega}^{+}$, for all $t \in[0, T)$, and for all $\phi \in C_{b}\left(\mathbb{T}^{N} \times \mathbb{R}\right)$,

$$
\frac{1}{\varepsilon} \int_{t}^{t+\varepsilon} \iint_{\mathbb{T}^{N} \times \mathbb{R}} \phi(x, \xi) \mathrm{d} \tilde{v}_{x, s}(\xi) \mathrm{d} x \mathrm{~d} s \rightarrow \iint_{\mathbb{T}^{N} \times \mathbb{R}} \phi(x, \xi) \mathrm{d} \tilde{v}_{x, t}^{+}(\xi) \mathrm{d} x .
$$

Since $T$ is arbitrary, we can as well work on $[0, T+1]$, instead of $[0, T]$. In that way, we can give a meaning to $\tilde{v}_{x, t}^{+}$for $t=T$ also. By (4.28) and (4.32), we have $\tilde{f}^{+}(x, t, \xi)=\tilde{f}(x, t, \xi)$ and $\tilde{v}_{x, t}^{+}=\tilde{v}_{x, t}$ for all $\tilde{\omega} \in \tilde{\Omega}^{+}$, for all $t \in(0, T) \backslash N_{0}$, for almost every $(x, \xi) \in \mathbb{T}^{N} \times \mathbb{R}$. If $\phi \in C_{b}(\mathbb{R})$ and $\tilde{\omega} \in \tilde{\Omega}^{+}$, then $(x, t) \mapsto\left\langle\tilde{v}_{x, t}, \phi\right\rangle$ is measurable and $(x, t) \mapsto\left\langle\tilde{v}_{x, t}^{+}, \phi\right\rangle$ differs from the latter function on a negligible subset of $\mathbb{T}^{N} \times(0, T)$. Therefore $(x, t) \mapsto\left\langle\tilde{v}_{x, t}^{+}, \phi\right\rangle$ itself is measurable. We deduce that $\tilde{v}^{+}$and $\tilde{f}^{+}$satisfy the measurability properties of a random Young measure and a random kinetic function respectively, and point 1 of the proposition is proved. The point 2 of the proposition follows from (4.32), which gives $\langle\tilde{f}(t), \varphi\rangle=F_{\varphi}(t)$ for all $t$. We have established item 7 of the proposition for $\varphi \in \Gamma$ only. By density the results remain true for all $\varphi \in C_{c}\left(\mathbb{T}^{N} \times \mathbb{R}\right)$. To obtain the last point of the proposition, we note first that $\tilde{v}^{+}$, like $\tilde{v}$, satisfies (4.11). If

$$
\sup _{J \subset[0, T]} \frac{1}{|J|} \int_{J} \int_{\mathbb{T}^{N}} \int_{\mathbb{R}}|\xi|^{p} \mathrm{~d} \tilde{\nu}_{x, t}^{+}(\xi) \mathrm{d} x \mathrm{~d} t<+\infty
$$

which happens $\tilde{\mathbb{P}}$-almost surely, then

$$
\sup _{J \subset[0, T]} \frac{1}{|J|} \int_{J} \int_{\mathbb{T}^{N}} \int_{\mathbb{R}}|\xi|^{p} \mathrm{~d} \tilde{v}_{x, t}^{+}(\xi) \mathrm{d} x \mathrm{~d} t=\sup _{t \in[0, T]} \int_{\mathbb{T}^{N}} \int_{\mathbb{R}}|\xi|^{p} \mathrm{~d} \tilde{v}_{x, t}^{+}(\xi) \mathrm{d} x
$$

by right-continuity of $t \mapsto \tilde{v}_{t}^{+}$. This gives the desired result.

We will now consider only the càdlàg versions. We replace $\tilde{v}$ by $\tilde{v}^{+}$and $\tilde{f}$ by $\tilde{f}^{+}$. This amounts to a modification on a negligible set. Therefore, this does not affect the results 1, 2, 4, 5, 6 in Theorem 4.6. We have now also items 7 and 8 of the theorem. It remains to define the filtration $\left(\tilde{\mathcal{F}}_{t}\right)$, the Wiener processes $\tilde{\beta}_{k}$ and to prove the points 3 and 9 of the theorem. We define $\left(\tilde{\mathcal{F}}_{t}\right), \tilde{\beta}_{k}$ and show item 3 in the proof of convergence of the stochastic integral in the next section 4.5.3. The equation (4.23) is established in Section 4.5.4. To finish the current section, let us first record the fact that (4.28) is now true for all $t$, due to our re-definition of $\tilde{f}$ and to (4.32), for $\tilde{\mathbb{P}}$-almost surely,

for all $t \in[0, T)$, for all $\varphi \in \Gamma,\langle\tilde{f}(t), \varphi\rangle=\left\langle f_{0}, \varphi\right\rangle+\tilde{J}_{\varphi}(t)-\left\langle\tilde{m}, \partial_{\xi} \varphi\right\rangle([0, t])$.

We deduce from (4.34) the following lemma:

Proposition 4.9. There exists a countable subset $\tilde{B} \subset[0, T]$ such that, $\tilde{\mathbb{P}}$-almost surely, for all $t \in[0, T] \backslash \tilde{B}$, for all $\varphi \in C_{c}\left(\mathbb{T}^{N} \times \mathbb{R}\right),\left\langle\tilde{f}^{n}(t), \varphi\right\rangle \rightarrow\langle\tilde{f}(t), \varphi\rangle$. 
Proof of Proposition 4.9. It is sufficient to obtain the convergence for $\varphi \in \Gamma$. We apply Lemma 2.1. Let

$$
\tilde{B}=\{t \in[0, T] ; \tilde{\mathbb{P}}(\pi \# \tilde{m}(\{t\})>0)>0\} .
$$

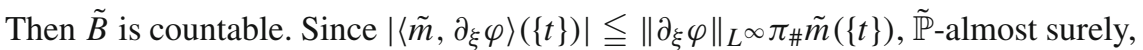
we have $\left\langle\tilde{m}, \partial_{\xi} \varphi\right\rangle(\{t\})=0$ for all $t \in[0, T] \backslash \tilde{B}$. For $t \in[0, T] \backslash \tilde{B}$ then, the righthand side of (4.27) is converging to the right-hand side of (4.34). We deduce the convergence of the left-hand sides, that is $\left\langle\tilde{f}^{n}(t), \varphi\right\rangle \rightarrow\langle\tilde{f}(t), \varphi\rangle$.

\subsubsection{Identification of the Limit: Convergence of the Stochastic Integral Let} us set

$$
M_{\varphi}^{*}(t)=\sum_{k \geqq 1} \int_{0}^{t} \int_{\mathbb{T}^{N}} \int_{\mathbb{R}} g_{k}(x, \xi) \varphi(x, \xi) d \tilde{\nu}_{x, s}(\xi) \mathrm{d} x d \tilde{\beta}_{k}(s),
$$

( $\tilde{\beta}_{k}$ is defined in Lemma 4.11 below). Our aim is to prove the identification $\{\tilde{M}\}=$ $\left\{M^{*}\right\}$. To obtain this result, we will use the martingale characterization developed in Section 4. The proof is decomposed into several steps.

Step 1. Filtration The approximation procedures to (1.1) (vanishing viscosity method, Finite Volume method as here) construct approximate solutions on arbitrary time intervals $[0, T]$. We will therefore consider the functions as defined on the whole time interval $\mathbb{R}_{+}$. This is simply to avoid the special case of the final time in the definition of the Skorokhod space $D([0, T]), c f$. [6], [30, Remark 1.10, p. 326]. Let $E$ be a Polish space. Let us introduce the following notations (see [30, Definition 1.1 p. 325$]$ in the case $\left.E=\mathbb{R}^{m}\right)$ : on the space $D\left(\mathbb{R}_{+} ; E\right), \mathscr{D}_{t}^{0}(E)$ is the $\sigma$-algebra generated by the maps $\alpha \mapsto \alpha(s), s \leqq t$ and

$$
\mathscr{D}_{t}(E)=\bigcap_{t<s} \mathscr{D}_{s}^{0}(E), \quad \mathscr{D}_{t-}(E)=\bigvee_{s<t} \mathscr{D}_{S}(E) .
$$

Note that $\mathscr{D}_{t}(E) \neq \mathscr{D}_{t}^{0}(E)$. Indeed, the time of entrance in an open subset $U$ of $E$,

$$
\tau_{U}(\alpha)=\inf \{t \geqq 0 ; \alpha(t) \in U\},
$$

is a stopping time with respect to $\left(\mathscr{D}_{t}(E)\right)$, but not with respect to $\left(\mathscr{D}_{t}^{0}(E)\right)[42$, Proposition I.4.6].

Proposition 4.10. Let $t>0$. Given a continuous bounded function $\theta: E \rightarrow \mathbb{R}$, $s \in[0, t)$ and $\varepsilon>0$, let $\theta_{\# s}$ denote the evaluation map $\alpha \mapsto \theta(\alpha(s))$ on $D\left(\mathbb{R}_{+} ; E\right)$, and let $\theta_{\# s}^{\varepsilon}$ denote the regularization

$$
\theta_{\# s}^{\varepsilon}: \alpha \rightarrow \frac{1}{\varepsilon} \int_{s}^{t \wedge(s+\varepsilon)} \theta(\alpha(\sigma)) \mathrm{d} \sigma
$$

of $\theta_{\# s}$. Then $\theta_{\# s}^{\varepsilon}$ is a $\mathscr{D}_{t-}(E)$-measurable bounded function, continuous for the Skorokhod topology. Let $\mathcal{H}$ denote the set of functions

$$
H=\theta_{\# s_{1}}^{1, \varepsilon_{1}} \cdots \theta_{\# s_{k}}^{k, \varepsilon_{k}},
$$


where $k \geqq 1,0 \leqq s_{1}<\cdots<s_{k}<t, 0<\varepsilon_{1}, \ldots, \varepsilon_{k}, \theta^{1}, \ldots, \theta^{k} \in C_{b}(E)$. Then every characteristic function $\mathbf{1}_{A}$ of a cylindrical set $A \in \mathscr{D}_{t-}(E)$ of the form

$$
A=\left\{\alpha \in D\left(\mathbb{R}_{+} ; E\right) ; \alpha\left(\tau_{1}\right) \in B_{1}, \ldots, \alpha\left(\tau_{k}\right) \in B_{k}\right\},
$$

for $B_{1}, \ldots, B_{k}$ closed subsets of $E$ and $0 \leqq \tau_{1}<\cdots<\tau_{k}<t$, is the bounded pointwise limit of a sequence of functions in $\mathcal{H}$.

Proof of Proposition 4.10. This is essentially the proof of [30, Lemma 1.45 p. 335]. Let $\alpha \in D\left(\mathbb{R}_{+} ; E\right)$ and let $\left(\alpha_{n}\right)$ be a sequence in $D\left(\mathbb{R}_{+} ; E\right)$ such that $\alpha_{n} \rightarrow \alpha$ almost everywhere on $[0, t]$; this is the case if $\alpha_{n} \rightarrow \alpha$ in $D\left(\mathbb{R}_{+} ; E\right)$ since $\alpha_{n}(\sigma) \rightarrow \alpha(\sigma)$ for every $\sigma$ not in the (countable) jump set of $\alpha$. Then, by the dominated convergence theorem, $\theta_{\# S}^{\varepsilon}\left(\alpha_{n}\right) \rightarrow \theta_{\# s}^{\varepsilon}(\alpha)$. Therefore $\theta_{\# s}^{\varepsilon}$ is a bounded function, continuous for the Skorokhod topology. It is $\mathscr{D}_{t-}(E)$-measurable since it is the bounded pointwise limit when $\eta \rightarrow 0$ of the sequence of $\mathscr{D}_{t-}(E)$-measurable functions

$$
\alpha \rightarrow \frac{1}{\varepsilon} \int_{s}^{(t-\eta) \wedge(s+\varepsilon)} \theta(\alpha(\sigma)) \mathrm{d} \sigma .
$$

Let us prove the last point. We can choose some sequences of continuous bounded functions $\theta_{1}^{n}, \ldots, \theta_{k}^{n}: E \rightarrow \mathbb{R}$ converging pointwise to the characteristic functions $\mathbf{1}_{B_{1}}, \ldots, \mathbf{1}_{B_{k}}$ (by considering, for example, the function distance to $B_{j}$, which is continuous). Since $\theta_{\# s}^{\varepsilon}$ is approaching $\theta_{\# s}$ for the bounded pointwise convergence, the result follows.

Remark 4.1. Note that the function $H$ defined by (4.38) is more than merely continuous for the Skorokhod topology. Indeed, what we have seen in the proof of Proposition 4.10 is that, for any $\alpha \in D\left(\mathbb{R}_{+} ; E\right)$ and any sequence $\left(\alpha_{n}\right)$ in $D\left(\mathbb{R}_{+} ; E\right)$ such that $\alpha_{n} \rightarrow \alpha$ almost everywhere on $[0, t]$, we have $H\left(\alpha_{n}\right) \rightarrow H(\alpha)$.

Let us set

$$
\{\tilde{f}\}=\left(\left\langle\tilde{f}, \varphi_{j}\right\rangle\right)_{j \in \mathbb{N}}, \quad E=\mathbb{R}^{\infty} \times \mathbb{R}^{\infty} \times \mathfrak{U}
$$

Recall that $\mathbb{R}^{\infty}$ is the product space $\prod_{\varphi \in \Gamma} \mathbb{R}$ endowed with the topology of pointwise convergence. Since $E$ is a product of Polish spaces, it is a Polish space. Since the product of $D\left(\mathbb{R}_{+} ; \mathbb{R}^{\infty}\right)$ with $C\left(\mathbb{R}_{+} ; \mathbb{R}^{\infty} \times \mathfrak{U}\right)$ is, topologically, a subset of $D\left(\mathbb{R}_{+} ; E\right)$, the triplet $(\{\tilde{f}\},\{\tilde{M}\}, \tilde{W})$ is an element of $D\left(\mathbb{R}_{+} ; E\right)$.

Definition 4.2. The filtration $\left(\tilde{\mathcal{F}}_{t}\right)$ is the completion of the filtration generated by the triplet $(\{\tilde{f}\},\{\tilde{M}\}, \tilde{W})$ as follows:

$$
\tilde{\mathcal{F}}_{t}=\sigma\left((\{\tilde{f}\},\{\tilde{M}\}, \tilde{W})^{-1}\left(\mathscr{D}_{t}(E)\right) \cup\{N \in \tilde{\mathcal{F}} ; \tilde{\mathbb{P}}(N)=0\}\right), \quad t \in[0, T] .
$$

Note that $\left(\tilde{\mathcal{F}}_{t}\right)$ is right-continuous since $\left(\mathscr{D}_{t}(E)\right)$ is, and complete by definition. 
Step 2. Wiener process Let $j: H \rightarrow \mathfrak{U}$ denote the injection of $H$ into $\mathfrak{U}$. Note that $j \circ j^{*}$ is a Trace-class operator on $\mathfrak{U}$. The Brownian motions $\tilde{\beta}_{k}(t)$ are the components of $\tilde{W}(t)$ on the orthonormal basis $\left(e_{k}\right)$.

Lemma 4.11. The process $\tilde{W}$ has a modification which is a $\left(\tilde{\mathcal{F}}_{t}\right)$-adapted $j \circ j^{*}$ Wiener process, and there exists a collection of mutually independent real-valued $\left(\tilde{\mathcal{F}}_{t}\right)$-Brownian motions $\left\{\tilde{\beta}_{k}\right\}_{k \geqq 1}$ such that

$$
\tilde{W}=\sum_{k \geqq 1} \tilde{\beta}_{k} e_{k}
$$

in $C([0, T] ; \mathfrak{U})$.

Note: see [12, Paragraph 4.1] for the definition of a $Q$-Wiener process.

Proof of Lemma 4.11. It is clear that $\tilde{W}$ is a $j \circ j^{*}$-cylindrical Wiener process (this notion is stable by convergence in law; actually it can be characterized in terms of the law of $\tilde{W}$ uniquely if we drop the usual hypothesis of a.s. continuity of the trajectories. This latter property of continuity can be recovered, after a possible modification of the process, by using Kolmogorov's Theorem). Also $\tilde{W}$ is $\left(\tilde{\mathcal{F}}_{t}\right)$ adapted by definition of the filtration $\left(\tilde{\mathcal{F}}_{t}\right)$. By [12, Proposition 4.1], we obtain the decomposition (4.41). The $\tilde{\mathbb{P}}$-a.s. convergence of the sum in (4.41) in the space $C([0, T] ; \mathfrak{U})$ is proved as in $[12$, Theorem 4.3].

Note that the last component $\tilde{W}^{n}$ of $\tilde{Z}^{n}$ depends a priori on $n$. Without loss of generality, we will replace $\tilde{W}^{n}$ by $\tilde{W}$. Of course, this does not affect the almost sure convergence of $\tilde{Z}^{n}$ to $\tilde{Z}$, and Lemma 4.11 asserts that this does not modify the law of $\tilde{Z}^{n}$. This operation is not mandatory for the validity of what follows, and quite natural since the original sequence $\left(Z^{n}\right)$ is stationary (as a sequence) with respect to its last argument.

Step 3. Martingales

Proposition 4.12. Let $\varphi_{j} \in \Gamma$. Let $\tilde{h}_{j, k}(t)$ be defined by

$$
\tilde{h}_{j, k}(t)=\int_{\mathbb{T}^{N}} \int_{\mathbb{R}} g_{k}(x, \xi) \varphi_{j}(x, \xi) \mathrm{d} \tilde{\nu}_{x, t}(\xi) \mathrm{d} x .
$$

Then, for $j \in \mathbb{N}, k \geqq 1$, the processes

$$
\tilde{M}_{j}(t), \quad \tilde{M}_{j}(t) \tilde{\beta}_{k}(t)-\int_{0}^{t} \tilde{h}_{j, k}(s) \mathrm{d} s, \quad\left|\tilde{M}_{j}(t)\right|^{2}-\int_{0}^{t}\left\|\tilde{h}_{j}(s)\right\|_{l^{2}\left(\mathbb{N}^{*}\right)}^{2} \mathrm{~d} s,
$$

and $(\tilde{W}(t))$ are $\left(\tilde{\mathcal{F}}_{t}\right)$-martingales.

Proof of Proposition 4.12. The proof is similar to the proof of [30, Proposition 1.1 p. 522], except that we do not use any hypothesis of boundedness here since we use the $\tilde{\mathbb{P}}$-almost sure convergence and the Vitali Theorem to pass to the limit in the expectation of the quantities of interest (an other minor difference with the proof 
of [30, Proposition 1.1 p. 522] is that $\tilde{M}$ is known to be continuous $\tilde{\mathbb{P}}$-a.s., not only càdlàg).

Let $t_{1}, t_{2} \in \mathbb{R}_{+}, t_{1}<t_{2}$ and let $H$ be a $\mathscr{D}_{t_{1}-}(E)$-measurable bounded function as in (4.38). By identities of the laws of $M_{\varphi}^{n}$ and $\tilde{M}_{\varphi}^{n}$, we have

$$
\tilde{\mathbb{E}}\left|\tilde{M}_{\varphi_{j}}^{n}\left(t_{2}\right)-\tilde{M}_{\varphi_{j}}^{n}\left(t_{1}\right)\right|^{2}=\mathbb{E}\left|M_{\varphi_{j}}^{n}\left(t_{2}\right)-M_{\varphi_{j}}^{n}\left(t_{1}\right)\right|^{2} .
$$

Using (4.21), it follows that

$$
\sup _{n} \tilde{\mathbb{E}}\left|H\left(\left\{\tilde{f}^{n}\right\},\left\{\tilde{M}^{n}\right\}, \tilde{W}\right)\left[\tilde{M}_{\varphi_{j}}^{n}\left(t_{2}\right)-\tilde{M}_{\varphi_{j}}^{n}\left(t_{1}\right)\right]\right|^{2}<+\infty,
$$

since $H$ is bounded. We have, in addition,

$$
\left(\left\{\tilde{f}^{n}\right\},\left\{\tilde{M}^{n}\right\}, \tilde{W}\right) \rightarrow(\{\tilde{f}\},\{\tilde{M}\}, \tilde{W})
$$

almost everywhere, $\tilde{\mathbb{P}}$-almost surely by Proposition 4.9, and thus,

$$
H\left(\left\{\tilde{f}^{n},\right\},\left\{\tilde{M}^{n}\right\}, \tilde{W}\right) \rightarrow H(\{\tilde{f}\},\{\tilde{M}\}, \tilde{W}),
$$

$\tilde{\mathbb{P}}$-almost surely. There is also convergence

$$
\tilde{M}_{\varphi_{j}}^{n}\left(t_{2}\right)-\tilde{M}_{\varphi_{j}}^{n}\left(t_{1}\right) \rightarrow \tilde{M}_{j}\left(t_{2}\right)-\tilde{M}_{j}\left(t_{1}\right)
$$

$\tilde{\mathbb{P}}$-almost surely. By Vitali's Theorem, we obtain

$$
\begin{aligned}
\tilde{\mathbb{E}} & {\left[H\left(\left\{\tilde{f}^{n},\right\},\left\{\tilde{M}^{n}\right\}, \tilde{W}\right)\left(\tilde{M}_{\varphi_{j}}^{n}\left(t_{2}\right)-\tilde{M}_{\varphi_{j}}^{n}\left(t_{1}\right)\right)\right] } \\
& \rightarrow \tilde{\mathbb{E}}\left[H(\{\tilde{f}\},\{\tilde{M}\}, \tilde{W})\left(\tilde{M}_{j}\left(t_{2}\right)-\tilde{M}_{j}\left(t_{1}\right)\right)\right] .
\end{aligned}
$$

By identities of the laws, the left-hand side of (4.44) is

$$
\mathbb{E}\left[H\left(\left\{f^{n},\right\},\left\{M^{n}\right\}, W\right)\left(M_{\varphi_{j}}^{n}\left(t_{2}\right)-M_{\varphi_{j}}^{n}\left(t_{1}\right)\right)\right]=0,
$$

since $M_{\varphi}^{n}$ is a $\left(\mathcal{F}_{t}\right)$-martingale. We deduce from (4.44) thus that

$$
\tilde{\mathbb{E}}\left[H(\{\tilde{f}\},\{\tilde{M}\}, \tilde{W})\left(\tilde{M}_{j}\left(t_{2}\right)-\tilde{M}_{j}\left(t_{1}\right)\right)\right]=0 .
$$

Due to Proposition 4.10, we deduce from (4.45) that

$$
\tilde{\mathbb{E}}\left[\mathbf{1}_{A}(\{\tilde{f}\},\{\tilde{M}\}, \tilde{W})\left(\tilde{M}_{j}\left(t_{2}\right)-\tilde{M}_{j}\left(t_{1}\right)\right)\right]=0
$$

for all cylindrical sets $A$ as in (4.39). The left-hand side of (4.46) defines a finite measure (due to (4.21)) which coincides with the trivial measure $A \mapsto 0$ for sets $A$ as in (4.39). Since such sets form a $\pi$-system which generates $\mathcal{D}_{t_{1}-}(E)$, hence a separating class, we deduce that (4.46) holds true for all $A \in \mathcal{D}_{t_{1}-}(E)$. It also follows then that $(4.45)$ is satisfied for all $\mathcal{D}_{t_{1}-}(E)$-measurable bounded function $H$. Now let $s, t \in[0, T)$ with $s<t$. Let $\left(s_{n}\right)$ and $\left(t_{n}\right)$ be some decreasing sequences in $\mathbb{R}_{+}$, converging to $s$ and $t$ respectively. Let $H$ be a $\mathscr{D}_{s}(E)$-measurable bounded 
function. Then $H$ is a $\mathscr{D}_{s_{n}-}\left(\mathbb{R}^{2+m}\right)$-measurable bounded function since $s<s_{n}$. By passing to the limit in (4.45) written with $t_{1}=s_{n}, t_{2}=t_{n}$ (we use the rightcontinuity of the processes her e), we obtain

$$
\tilde{\mathbb{E}}\left[H(\{\tilde{f}\},\{\tilde{M}\}, \tilde{W})\left(\tilde{M}_{j}(t)-\tilde{M}_{j}(s)\right)\right]=0 .
$$

This shows that $\left(\tilde{M}_{j}(t)\right)$ is a $\tilde{\mathcal{F}}_{t}$-martingale. The proof that $(\tilde{W}(t))$ is a $\tilde{\mathcal{F}}_{t}$-martingale is similar, we do not give the details of that point. To go on, let us now define the processes

$$
\tilde{H}_{j, k}^{n}(t)=\int_{0}^{t} \tilde{h}_{j, k}^{n}(s) \mathrm{d} s, \quad \tilde{H}_{j, k}(t)=\int_{0}^{t} \tilde{h}_{j, k}(s) \mathrm{d} s,
$$

and

$$
\tilde{\mathcal{H}}_{j}^{n}(t)=\int_{0}^{t}\left\|\tilde{h}_{j}^{n}(s)\right\|_{l^{2}\left(\mathbb{N}^{*}\right)}^{2} \mathrm{~d} s, \quad \tilde{\mathcal{H}}_{j}(t)=\int_{0}^{t}\left\|\tilde{h}_{j}(s)\right\|_{l^{2}\left(\mathbb{N}^{*}\right)}^{2} \mathrm{~d} s,
$$

and the processes

$$
\begin{cases}\tilde{Y}_{j, k}^{n}(t)=\tilde{M}_{j}^{n}(t) \tilde{\beta}_{k}(t)-\tilde{H}_{j, k}^{n}(t), & \tilde{Y}_{j, k}(t)=\tilde{M}_{j}(t) \tilde{\beta}_{k}(t)-\tilde{H}_{j, k}(t), \\ \tilde{V}_{j}^{n}(t)=\left|\tilde{M}_{j}^{n}(t)\right|^{2}-\tilde{\mathcal{H}}_{j}^{n}(t), & \tilde{V}_{j}(t)=\left|\tilde{M}_{j}(t)\right|^{2}-\tilde{\mathcal{H}}_{j}(t) .\end{cases}
$$

To complete the proof of Proposition 4.12, we have to show that $\left(\tilde{Y}_{k}(t)\right)$ and $\left(\tilde{V}_{j}(t)\right)$ are $\tilde{\mathcal{F}}_{t}$-martingale. We will use the following result:

Lemma 4.13. Let $T>0$. Then, up to a subsequence, for all $j \in \mathbb{N}, k \in \mathbb{N}^{*}, \tilde{\mathbb{P}}$ almost surely, $\tilde{h}_{j, k}^{n} \rightarrow \tilde{h}_{j, k}$ and $\left\|\tilde{h}_{j}^{n}(\cdot)\right\|_{l^{2}\left(\mathbb{N}^{*}\right)}^{2} \rightarrow\left\|\tilde{h}_{j}(\cdot)\right\|_{l^{2}\left(\mathbb{N}^{*}\right)}^{2}$ in $L^{1}(0, T)$, when $n \rightarrow+\infty$.

Lemma 4.13 implies that, $\tilde{\mathbb{P}}$-almost surely, for every $t \in[0, T], \tilde{H}_{j, k}^{n}(t)$ and $\tilde{\mathcal{H}}_{j}^{n}(t)$ are converging to $\tilde{H}_{j, k}(t)$ and $\tilde{\mathcal{H}}_{j}(t)$ respectively. We have also $\tilde{M}_{j}^{n} \rightarrow \tilde{M}_{j}$ in $C\left(\mathbb{R}_{+}\right)$, from which follows the convergences $\tilde{M}_{j}^{n} \tilde{\beta}_{k} \rightarrow \tilde{M}_{j} \tilde{\beta}_{k}$ and $\left|\tilde{M}_{j}^{n}\right|^{2} \rightarrow\left|\tilde{M}_{j}\right|^{2}$ in $C\left(\mathbb{R}_{+}\right), \tilde{\mathbb{P}}$-almost surely. We deduce that, $\tilde{\mathbb{P}}$-almost surely,

$$
\tilde{Y}_{j, k}^{n}(t) \rightarrow \tilde{Y}_{j, k}(t), \quad \tilde{V}_{j}^{n}(t) \rightarrow \tilde{V}_{j}(t),
$$

for all $t \geqq 0$. With the estimate (4.21), it is easy to obtain the bounds

$$
\tilde{\mathbb{E}}\left|\tilde{Y}_{j, k}^{n}(t)-\tilde{Y}_{j, k}^{n}(s)\right|^{2} \leqq C, \quad \tilde{\mathbb{E}}\left|\tilde{V}_{j}^{n}(t)-\tilde{V}_{j}^{n}(s)\right|^{2} \leqq C,
$$

where the constant $C$ depend on $s, t \in[0, T], k$, but not on $n$. By (4.48) and (4.49) (this last condition shows the equi-integrability of $\left(\tilde{Y}_{j, k}^{n}(t)-\tilde{Y}_{j, k}^{n}(s)\right)$ and $\left(\tilde{V}_{j}^{n}(t)-\tilde{V}_{j}^{n}(s)\right)$ respectively), we can use the arguments applied to the martingale $\tilde{M}_{\varphi}^{n}(t)$ in the first part of the proof; this it will establish that $\tilde{Y}_{j, k}(t)$ and $\tilde{V}_{j}(t)$ are $\left(\tilde{\mathcal{F}}_{t}\right)$-martingales. 
Let us now give the

Proof of Lemma 4.13. Let us first show that, for all $j, k$, we have the following convergence:

$$
\tilde{h}_{j, k}^{n} \rightarrow \tilde{h}_{j, k} \quad \text { in } \quad L^{2}((0, T) \times \tilde{\Omega})
$$

Define, for every $\psi \in C_{b}\left(\mathbb{T}^{N} \times \mathbb{R}\right)$,

$$
\tilde{h}_{\psi}^{n}(t)=\int_{\mathbb{T}^{N}} \int_{\mathbb{R}} \psi(x, \xi) \mathrm{d} \tilde{v}_{x, t}^{n}(\xi) \mathrm{d} x, \quad \tilde{h}_{\psi}(t)=\int_{\mathbb{T}^{N}} \int_{\mathbb{R}} \psi(x, \xi) \mathrm{d} \tilde{v}_{x, t}(\xi) \mathrm{d} x .
$$

If $\psi \in C_{c}^{1}\left(\mathbb{T}^{N} \times \mathbb{R}\right)$, then $\tilde{h}_{\psi}^{n}(t)=\left\langle\tilde{f}^{n}(t), \partial_{\xi} \psi\right\rangle$. By Proposition 4.9, we have, $\tilde{\mathbb{P}}$-almost surely,

$$
\text { for all } t \in[0, T] \backslash \tilde{B}, \tilde{h}_{\psi}^{n}(t) \rightarrow \tilde{h}_{\psi}(t)
$$

Using the Jensen inequality, we have

$$
\left\|\tilde{h}_{\psi}^{n}-\tilde{h}_{\psi}\right\|_{L^{2}((0, T) \times \tilde{\Omega})}^{2} \leqq 4 T\|\psi\|_{C_{b}\left(\mathbb{T}^{N} \times \mathbb{R}\right)}^{2} .
$$

By the Vitali Theorem, we obtain the convergence $\tilde{h}_{\psi}^{n} \rightarrow \tilde{h}_{\psi}$ in $L^{1}((0, T) \times \tilde{\Omega})$. Using (4.53) as well, we see that this convergence can be extended to the case of a general integrand $\psi \in C_{b}\left(\mathbb{T}^{N} \times \mathbb{R}\right)$. Let us then take $\psi=g_{k} \varphi_{j}$. We obtain first $\tilde{h}_{j, k}^{n} \rightarrow \tilde{h}_{j, k}$ in $L^{2}((0, T) \times \tilde{\Omega})$. It follows that, up to a subsequnce, $\tilde{\mathbb{P}}$-almost surely, $\tilde{h}_{j, k}^{n} \rightarrow \tilde{h}_{j, k}$ in $L^{2}(0, T)$, hence in $L^{1}(0, T)$. The subsequence and the $\tilde{\mathbb{P}}$-almost sure property can be made independent on $j, k$, since $\Gamma \times \mathbb{N}^{*}$ is countable. The growth hypothesis (1.3) also shows that

$$
\sum_{k}\left\|\tilde{h}_{j, k}^{n}-\tilde{h}_{j, k}\right\|_{L^{2}((0, T) \times \tilde{\Omega})}^{2} \leqq 4 D_{0}\left(1+C_{2}\right) T\left\|\varphi_{j}\right\|_{C_{b}\left(\mathbb{T}^{N} \times \mathbb{R}\right)}^{2} .
$$

Again, using the dominated convergence theorem, we deduce that

$$
\left\|\tilde{h}_{j}^{n}\right\|_{l^{2}\left(\mathbb{N}^{*}\right)}^{2} \rightarrow\left\|\tilde{h}_{j}\right\|_{l^{2}\left(\mathbb{N}^{*}\right)}^{2}
$$

in $L^{1}((0, T) \times \tilde{\Omega})$, which allows us to conclude the proof of the lemma.

Step 4. Conclusion of the martingale method Let us first prove that $M_{\varphi}^{*}(t)$ given in (4.36) is well-defined.

Lemma 4.14. Item 3 in Theorem 4.6 is satisfied, that is: for all $\psi \in C_{b}(\mathbb{R})$, $(x, t) \mapsto\left\langle\psi, \tilde{v}_{x, t}\right\rangle$ belongs to $L_{\tilde{\mathcal{P}}}^{2}\left(\mathbb{T}^{N} \times[0, T] \times \tilde{\Omega}\right)$. 
Proof of Lemma 4.14. For $\psi \in C_{b}(\mathbb{R})$, set $\tilde{X}_{\psi}(x, t)=\left\langle\psi, \tilde{v}_{x, t}\right\rangle$. We have $\tilde{X}_{\varphi} \in$ $L^{2}\left(\mathbb{T}^{N} \times[0, T] \times \tilde{\Omega}\right)$, with

$$
\tilde{\mathbb{E}}\left\|\tilde{X}_{\psi}\right\|_{L^{2}\left(\mathbb{T}^{N} \times[0, T]\right)}^{2} \leqq\|\psi\|_{C_{b}(\mathbb{R})}^{2} T .
$$

If $\theta \in C\left(\mathbb{T}^{N}\right)$, and if $\psi$ is $C^{1}$, vanishes in the neighbourhood of $-\infty$ and satisfies $\psi^{\prime} \in C_{c}(\mathbb{R})$, then, due to $(2.31)$, we have

$$
\left\langle\tilde{X}_{\psi}(t), \theta\right\rangle_{L^{2}\left(\mathbb{T}^{N}\right)}=\langle\tilde{f}(t), \varphi\rangle, \quad \varphi(x, \xi):=\theta(x) \psi^{\prime}(\xi) .
$$

By Item 7 of Theorem 4.6, the process $Y_{t}:=\left\langle\tilde{X}_{\psi}(t), \theta\right\rangle_{L^{2}\left(\mathbb{T}^{N}\right)}$ is càdlàg. Since $\left(Y_{t}\right)$ is adapted by definition of $\left(\tilde{\mathcal{F}}_{t}\right)$, it is an optional process [42, p. 172]. In particular, $\left(Y_{t}\right)$ is progressively measurable [42, Proposition 4.8], hence $Y \in L_{\tilde{\mathcal{P}}}^{2}([0, T] \times$ $\tilde{\Omega}$ ). A limiting argument (by approximation and truncation of the function $\psi$ in particular), using (4.54) and the fact that $\tilde{v}$ vanishes at infinity shows that the result holds true when $\psi$ is merely a function in $C_{b}(\mathbb{R})$ and $\theta$ any function in $L^{2}\left(\mathbb{T}^{N}\right)$. We obtain, therefore, that, for all $\psi \in C_{b}(\mathbb{R}), X_{\psi}$ belongs to $L_{\tilde{\mathcal{P}}}^{2}([0, T] \times$ $\tilde{\Omega} ; L^{2}\left(\mathbb{T}^{N}\right)$ - weak). Since being weakly or strongly $\tilde{\mathbb{P}}$-measurable is the same thing, (cf. Section 2.1.1), we have established the result.

We can apply now Proposition 4.2. Indeed, due to Lemma 4.14, the processes $\tilde{h}_{j, k}$ in Proposition 4.12 are in $L_{\tilde{\mathcal{P}}}^{2}([0, T] \times \tilde{\Omega})$. By the martingale property (4.42), we conclude that $\tilde{M}_{\varphi}(t)=M_{\varphi}^{*}(t)$, with $M_{\varphi}^{*}(t)$ defined by (4.36), for every $\varphi \in \Gamma$.

4.5.4. Identification of the Limit: Equation We now prove (4.23). Let $\varphi \in \Gamma$. By item 2 and item 6 of Theorem 4.6, using also the identity $\tilde{M}_{\varphi}(t)=M_{\varphi}^{*}(t)$, we have the identification

$$
\begin{aligned}
\tilde{J}_{\varphi}(t)= & \int_{0}^{t}\langle\tilde{f}(s), a(\xi) \cdot \nabla \varphi\rangle \mathrm{d} s+\sum_{k \geqq 1} \int_{0}^{t} \int_{\mathbb{T}^{N}} \int_{\mathbb{R}} g_{k}(x, \xi) \varphi(x, \xi) \mathrm{d} \tilde{v}_{x, s}(\xi) \mathrm{d} x \mathrm{~d} \tilde{\beta}_{k}(s) \\
& +\frac{1}{2} \int_{0}^{t} \int_{\mathbb{T}^{N}} \int_{\mathbb{R}} \partial_{\xi} \varphi(x, \xi) \mathbf{G}^{2}(x, \xi) \mathrm{d} \tilde{v}_{x, s}(\xi) \mathrm{d} x \mathrm{~d} s .
\end{aligned}
$$

The equation (4.23) follows therefore from the identity (4.34).

\subsection{Pathwise Solutions and Almost Sure Convergence}

If $f_{0}$ is at equilibrium in Theorem 4.6, then we have seen in Theorem 3.2 that (1.1) admits a unique solution for a given initial datum. We can use this uniqueness result to obtain the existence of a pathwise solution and convergence in $L^{p}$ of the sequence of approximate solutions in that case.

Theorem 4.15. (Pathwise solution). Suppose that there exists a sequence of approximate generalized solutions $\left(f^{n}\right)$ to $(1.1)$ with initial datum $f_{0}^{n}$ satisfying (4.10), (4.14) and the tightness condition (4.15) and such that $\left(f_{0}^{n}\right)$ converges to the equilibrium function $\mathrm{f}_{0}(\xi)=\mathbf{1}_{u_{0}>\xi}$ in $L^{\infty}\left(\mathbb{T}^{N} \times \mathbb{R}\right)$-weak-*, where $u_{0} \in L^{\infty}\left(\mathbb{T}^{N}\right)$. We then have 
1. there exists a unique solution $u \in L^{1}\left(\mathbb{T}^{N} \times[0, T] \times \Omega\right)$ to (1.1) with initial datum $u$;

2. let

$$
u^{n}(x, t)=\int_{\mathbb{R}} \xi \mathrm{d} v_{x, t}^{n}(\xi)=\int_{\mathbb{R}}\left(f^{n}(x, t, \xi)-\mathbf{1}_{0>\xi}\right) \mathrm{d} \xi .
$$

Then, for all $p \in\left[1, \infty\left[,\left(u^{n}\right)\right.\right.$ is converging to $u$ with the following two different modes of convergence: $u_{n} \rightarrow u$ in $L^{p}\left(\mathbb{T}^{N} \times(0, T) \times \Omega\right)$ and, for a subsequence $\left(n_{k}\right)$, almost surely, for all $t \in[0, T], u^{n_{k}}(t) \rightarrow u(t)$ in $L^{p}\left(\mathbb{T}^{N}\right)$.

Proof of Theorem 4.15. We use the Gyöngy-Krylov argument, [24, Lemma 1.1] (the basis of the Gyöngy-Krylov argument is this simple fact: if a couple $\left(X_{n}, Y_{n}\right)$ of random variables converges in law to a random variable written $(Z, Z)$, that is concentrated on the diagonal, then $X_{n}-Y_{n}$ converges to 0 in probability). Let us go back to Section 4.5.1. We introduce the random variable

$Z^{n, q}=\left(v^{n},\left\{J^{n}\right\},\left\{M^{n}\right\},\left\{\varepsilon^{n}\right\},\left\{f_{\text {in }}^{n}\right\}, \mu^{n},\left\|m^{n}\right\|, v^{q},\left\{J^{q}\right\},\left\{M^{q}\right\},\left\{\varepsilon^{q}\right\},\left\{f_{\text {in }}^{q}\right\}, \mu^{q},\left\|m^{q}\right\|, W\right)$

in the state space $\mathcal{Z}$ equal to

$$
\begin{aligned}
\mathcal{Y}^{1} & \times \mathcal{E} \times \mathcal{P}^{1}\left(\mathbb{T}^{N} \times[0, T] \times \mathbb{R}\right) \times \mathbb{R}_{+} \times \mathcal{Y}^{1} \times \mathcal{E} \times \mathcal{P}^{1}\left(\mathbb{T}^{N} \times[0, T] \times \mathbb{R}\right) \\
& \times \mathbb{R}_{+} \times \mathcal{X}_{W} .
\end{aligned}
$$

We repeat the arguments used in Section 4.5 to show that $Z^{n, q}$ is tight in $\mathcal{Z}$ and that there exists a probability space $(\tilde{\Omega}, \tilde{\mathcal{F}}, \tilde{\mathbb{P}})$ and a new random variable $\tilde{Z}^{n, q}$ with the same law as $Z^{n, q}$, such that a subsequence $\left(\tilde{Z}^{n_{l}, q_{l}}\right)_{l}$ is converging $\tilde{\mathbb{P}}$-almost surely in $\mathcal{Z}$ to a random variable $\tilde{Z}$. Let $\tilde{v}$ be the the first component of $\tilde{Z}$ and $\tilde{\tilde{v}}$ be the seventh component of $\tilde{Z}$. Repeating all steps from Section 4.5.2, 4.5.3, 4.5.4, we obtain the generalized solutions

$$
\tilde{f}(x, t, \xi)=\tilde{v}_{(x, t)}(\xi,+\infty) \text { and } \tilde{\tilde{f}}(x, t, \xi)=\check{\tilde{v}}_{(x, t)}(\xi,+\infty),
$$

to Equation (1.1) with probabilistic data $\left(\tilde{\Omega}, \tilde{\mathcal{F}}, \tilde{\mathbb{P}},\left(\tilde{\mathcal{F}}_{t}\right), \tilde{W}\right)$, where $\left(\tilde{\mathcal{F}}_{t}\right)$ is the completion of the filtration generated by the five-uplet $(\{\tilde{f}\},\{\tilde{M}\},\{\tilde{\tilde{f}}\},\{\tilde{\tilde{M}}\}, \tilde{W})$ $\tilde{\mathcal{F}}_{t}=\sigma\left((\{\tilde{f}\},\{\tilde{M}\},\{\tilde{\tilde{f}}\},\{\tilde{\tilde{M}}\}, \tilde{W})^{-1}\left(\mathscr{D}_{t}(E) \times \mathscr{D}_{t}(\check{E})\right) \cup\{N \in \tilde{\mathcal{F}} ; \tilde{\mathbb{P}}(N)=0\}\right)$ for $t \in[0, T]$, with

$$
E:=\mathbb{R}^{\infty} \times \mathbb{R}^{\infty}, \quad \check{E}:=\mathbb{R}^{\infty} \times \mathbb{R}^{\infty} \times \mathfrak{U}
$$

Note that $\mathscr{D}_{t}(E) \times \mathscr{D}_{t}(\check{E}) \neq \mathscr{D}_{t}(E \times \check{E})$, since the natural topologies of $D\left(\mathbb{R}_{+} ; E\right) \times$ $D\left(\mathbb{R}_{+} ; \check{E}\right)$ and $D\left(\mathbb{R}_{+} ; E \times \check{E}\right)$ are different (the topology of the former is the product topology of the Skorokhod topologies on each space; this authorizes two changes of times, one for each coordinate; for the Skorokhod topology on $D\left(\mathbb{R}_{+} ; E \times \check{E}\right)$, only one change of time is admissible). The solutions $\tilde{f}$ and $\tilde{\tilde{f}}$ have the same initial condition $f_{0}$, which is an equilibrium function $\mathrm{f}_{0}$. By Theorem 3.2, we have

$$
\tilde{f}=\check{\tilde{f}}=\mathrm{f},
$$


where $\mathrm{f}$ is the equilibrium function $\mathbf{1}_{\tilde{u}>\xi}$, where

$$
\tilde{u}(x, t):=\int_{\mathbb{R}} \xi \mathrm{d} \tilde{v}_{(x, t)}(\xi) .
$$

A first consequence of (4.55) is that $\tilde{v}=\tilde{\tilde{v}}$, $\tilde{\mathbb{P}}$-almost surely. By Remark 3.2 on the uniqueness of the kinetic measure, we have also $\tilde{m}=\tilde{\tilde{m}}$, $\tilde{\mathbb{P}}$-almost surely. We apply the Gyöngy-Krylov argument: we obtain that $\left(v^{n}\right)$ is converging in probability in $\mathcal{Y}^{1}$ and $\left(m^{n}\right)$ is converging in probability in $\mathcal{M}_{b}\left(\mathbb{T}^{N} \times[0, T] \times \mathbb{R}\right)$-weak-*. Extracting an additional subsequence if necessary, we can assume that the convergences are also $\mathbb{P}$-almost sure. By the arguments of the sections 4.5.2, 4.5.3, 4.5.4, it follows that $f(t, x, \xi):=v_{x, t}(\xi,+\infty)$ is a generalized solution to (1.1). Note, to give few details, that we do not need to follow Step 1 and Step 2 of Section 4.5.3 here, since the filtration $\left(\mathcal{F}_{t}\right)$ and the Wiener processes $\beta_{k}(t)$ are already known here. The convergence of the stochastic integral in $J_{\varphi}^{n}(t)$ does not require the martingale method of Step 3. of Section 4.5.3 either. Using the $L^{2}$ convergence of the integr and ( $c f$. Lemma 4.13) is sufficient by the Itô isometry.

We use the second identity in (4.55) now. This states, equivalently, that $\tilde{\mathbb{P}}$-almost surely, for almost every $(x, t), \tilde{v}_{(x, t)}=\delta_{\tilde{u}(x, t)}$. The fact that $\tilde{v}$ is a Dirac mass can be characterized in terms of equality in the Jensen Inequality:

$$
\tilde{\mathbb{E}} \iint_{\mathbb{T}^{N} \times(0, T)} \Phi\left(\int_{\mathbb{R}} \xi \mathrm{d} \tilde{v}_{(x, t)}(\xi)\right) \mathrm{d} x \mathrm{~d} t=\tilde{\mathbb{E}} \iint_{\mathbb{T}^{N} \times(0, T)} \int_{\mathbb{R}} \Phi(\xi) \mathrm{d} \tilde{v}_{(x, t)}(\xi) \mathrm{d} x \mathrm{~d} t,
$$

where $\Phi$ is a strictly convex, polynomially bounded function, like $\Phi(\xi)=\xi^{2}$ for example. The identity (4.56) depends on $\operatorname{Law}(\tilde{v})=\operatorname{Law}(v)$ uniquely. Therefore $v$ also is almost surely a Dirac mass; $\mathbb{P}$-almost surely, for almost every $(x, t)$, $v_{(x, t)}=\delta_{u(x, t)}$, where

$$
u(x, t):=\int_{\mathbb{R}} \xi \mathrm{d} v_{(x, t)}(\xi) .
$$

(Remark that $v_{(x, t)}=\delta_{u(x, t)}$ a.s., almost everywhere, is also a consequence of Theorem 3.2. However this theorem is difficult to show, and, although we have already used Theorem 3.2, the argument based on (4.56) is simple and natural.) By Proposition 2.8, $u$ is a solution to (1.1); it is the unique solution by Theorem 3.2. Using Lemma 2.6 as well, we have $\left\|u^{n}-u\right\|_{L^{p}\left(\mathbb{T}^{N} \times(0, T)\right)}^{p} \rightarrow 0$ in probability. We also have the uniform bound

$$
\mathbb{E}\left\|u^{n}-u\right\|_{L^{p}\left(\mathbb{T}^{N} \times(0, T)\right)}^{p r} \leqq C,
$$

where $r>1$ and $C$ is independent on $n$. Taking (4.57) for granted, we deduce, with the convergence in probability, that $\mathbb{E}\left\|u^{n}-u\right\|_{L^{p}\left(\mathbb{T}^{N} \times(0, T)\right)}^{p} \rightarrow 0$ and we obtain the first part of the second point of Theorem 4.15. The bound (4.57) follows from the estimate

$$
\mathbb{E}\left\|u^{n}-u\right\|_{L^{p}\left(\mathbb{T}^{N} \times(0, T)\right)}^{p r} \leqq \mathbb{E}\left\|u^{n}-u\right\|_{L^{p r}\left(\mathbb{T}^{N} \times(0, T)\right)}^{p r} T^{r-1},
$$


as well as (2.5) and (4.10). To prove this almost surely, for all $t \in[0, T], u_{n_{k}}(t) \rightarrow$ $u(t)$ in $L^{p}\left(\mathbb{T}^{N}\right)$, we use Lemma 2.6 and Proposition 4.9. This gives, almost surely, for all $t \in[0, T] \backslash B_{\text {at }}, u_{n_{k}}(t) \rightarrow u(t)$ in $L^{p}\left(\mathbb{T}^{N}\right)$, where $B_{\text {at }}$ is defined in Lemma 2.1. Since, almost surely, $u$ is continuous in time with values in $L^{p}\left(\mathbb{T}^{N}\right)$ by Corollary 3.3, it follows from (2.34) that $B_{\text {at }}$ is empty. This gives the desired result.

\section{Some Applications}

\subsection{Vanishing Viscosity Method}

Assume that (1.3) and (1.4) are satisfied. Consider the parabolic approximation to $(1.1)$ :

$$
\mathrm{d} u^{\eta}+\operatorname{div}\left(A\left(u^{\eta}\right)\right) \mathrm{d} t-\eta \Delta u^{\eta} \mathrm{d} t=\Phi^{\eta}\left(x, u^{\eta}\right) \mathrm{d} W(t) .
$$

For $\eta>0$ and $u_{0}^{\eta} \in L^{\infty}\left(\mathbb{T}^{N}\right)$, the existence of solutions to (5.1) under the initial condition $u^{\eta}(0)=u_{0}^{\eta}$ has been proved in [25] provided the noise has a finite number of components. Therefore, we assume [compare to (1.2)]

$$
\Phi^{\eta}(x, u)=\sum_{1 \leqq k \leqq K_{\eta}} g_{k}(x, u) e_{k},
$$

where $K_{\eta}$ is finite, $K_{\eta} \rightarrow+\infty$ when $\eta \rightarrow 0$. Let $\left(\eta_{n}\right) \downarrow 0$. In [14], we have shown that the sequence $\left(u^{\eta_{n}}\right)$ gives rise to a sequence of approximate generalized solutions $\left(f^{n}\right)$, with random measure $m^{n}$, given by

$$
\begin{aligned}
f^{n} & =\mathrm{f}^{n}=\mathbf{1}_{u^{\eta_{n}}>\xi}, \\
\left\langle m^{n}, \varphi\right\rangle & =\iint_{\mathbb{T}^{N} \times(0, T)} \varphi(x, t) \eta_{n}\left|\nabla_{x} u^{\eta_{n}}(x, t)\right|^{2} \mathrm{~d} x \mathrm{~d} t, \\
\varepsilon_{n}(t, \varphi) & =\eta_{n} \int_{0}^{t} \int_{\mathbb{T}^{N}} \mathrm{f}^{n}(x, s, \xi) \Delta \varphi(x, \xi) \mathrm{d} \xi \mathrm{d} x \mathrm{~d} s .
\end{aligned}
$$

Here the order is $\mathrm{d}=2$. Let $p \in[1,+\infty)$. By Theorem 4.15 , we recover the result given in [14] of convergence $u^{\eta} \rightarrow u$ in $L^{p}\left(\mathbb{T}^{N} \times(0, T) \times \Omega\right)$, where $u$ is the solution to (1.1) with initial datum $u_{0}$. We also obtain that, if $\left(\eta_{n}\right) \downarrow 0$, then, for a

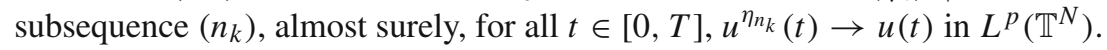

\subsection{BGK Approximation}

We consider now the following BGK approximation to (1.1):

$$
\begin{aligned}
\mathrm{d} f^{\eta}+a(\xi) \cdot \nabla_{x} f^{\eta} \mathrm{d} t & =\frac{\mathrm{f}^{\eta}-f^{\eta}}{\eta} \mathrm{d} t-\partial_{\xi} f^{\eta} \Phi^{\eta} \mathrm{d} W(t)-\frac{1}{2} \partial_{\xi}\left(\mathbf{G}^{2} \partial_{\xi}\left(-f^{\eta}\right)\right), \\
\mathrm{f}^{\eta} & :=\mathbf{1}_{u^{\eta}>\xi}, \quad u^{\eta}=\int_{\mathbb{R}}\left(f^{\eta}(\xi)-\mathbf{1}_{0>\xi}\right) \mathrm{d} \xi
\end{aligned}
$$


Assume (5.2), and assume that (1.4) is satisfied and that (instead of (1.3)), either $\mathbf{G}^{2}(x, \xi) \leqq D_{0}\left|\xi^{2}\right|$ or $\mathbf{G}^{2}(x, \xi) \leqq D_{0}$ is satisfied. M. Hofmanová has proved in [27] the existence of solutions to (5.3)-(5.4) with given initial datum $f_{0}^{\eta}=\mathrm{f}_{0}^{\eta}=\mathbf{1}_{u_{0}^{\eta}>\xi}$ (the fact that the initial datum is at equilibrium can be relaxed). Let $\left(\eta_{n}\right) \downarrow 0$. Then $f^{n}:=f^{\eta_{n}}$ provides a sequence of generalized approximate solutions of order $\mathrm{d}=0$, with

$$
\begin{aligned}
\partial_{\xi} m^{n} & =\frac{\mathrm{f}^{n}-f^{n}}{\eta_{n}}, \\
\varepsilon_{n}(t, \varphi) & =0 .
\end{aligned}
$$

Let $u_{0} \in L^{\infty}\left(\mathbb{T}^{N}\right)$. Assume $u_{0}^{\eta_{n}} \rightarrow u_{0}$ in $L^{p}\left(\mathbb{T}^{N}\right)$ for all $p \in[1,+\infty)$, and let $u$ be the solution to (1.1) with initial datum $u_{0}$. By Theorem 4.15, we recover the convergence $u^{n} \rightarrow u$ proved in [27]. We also have, for a subsequence $\left(n_{k}\right)$, almost surely, that for all $t \in[0, T], u^{n_{k}}(t) \rightarrow u(t)$ in $L^{p}\left(\mathbb{T}^{N}\right)$

\subsection{Approximation by the Finite Volume Method}

The approximation of (1.1) by the Finite Volume method is considered in the companion paper [16].

\section{References}

1. Bauzet, C.: Time-splitting approximation of the Cauchy problem for a stochastic conservation law. Math. Comput. Simulation 118, 73-86 (2015)

2. Bauzet, C., Charrier, J., Gallouët, T.: Convergence of flux-splitting finite volume schemes for hyperbolic scalar conservation laws with a multiplicative stochastic perturbation. Math. Comp. 85(302), 2777-2813 (2016)

3. Bauzet, C., Charrier, J., Gallouët, T.: Convergence of monotone finite volume schemes for hyperbolic scalar conservation laws with multiplicative noise. Stoch. Partial Differ. Equ. Anal. Comput. 4(1), 150-223 (2016)

4. Bauzet, C., Vallet, G., Wittbold, P.: The Cauchy problem for conservation laws with a multiplicative stochastic perturbation. J. Hyperbolic Differ. Equ. 9(4), 661-709 (2012)

5. Bauzet, C., Vallet, G., Wittbold, P.: The Dirichlet problem for a conservation law with a multiplicative stochastic perturbation. J. Funct. Anal. 266(4), 2503-2545 (2014)

6. Billingsley, P.: Convergence of probability measures. Wiley Series in Probability and Statistics: Probability and Statistics. John Wiley \& Sons Inc., New York, second edition, 1999. A Wiley-Interscience Publication

7. BRZEŹNIAK, Z., ONDREJÁt, M.: Weak solutions to stochastic wave equations with values in Riemannian manifolds. Comm. Partial Differential Equations 36(9), 1624-1653 (2011)

8. Castaing, C., Raynaud de Fitte, P., Valadier, M.: Young measures on topological spaces, volume 571 of Mathematics and its Applications. Kluwer Academic Publishers, Dordrecht, 2004. With applications in control theory and probability theory

9. Chen, G.-Q., Ding, Q., Karlsen, K.H.: On nonlinear stochastic balance laws. Arch. Ration. Mech. Anal. 204(3), 707-743 (2012)

10. Chen, G.-Q., Perthame, B.: Well-posedness for non-isotropic degenerate parabolichyperbolic equations. Ann. Inst. H. Poincaré Anal. Non Linéaire 20(4), 645-668, (2003) 
11. Chung, K.L., Williams, R.J.: Introduction to stochastic integration. Probability and its Applications. Birkhäuser Boston, Inc., Boston, MA, second edition, 1990

12. Da Prato, G., ZавсZYк, J.: Stochastic equations in infinite dimensions, volume 44 of Encyclopedia of Mathematics and its Applications. Cambridge University Press, Cambridge, 1992

13. Debussche, A., Hofmanová, M., Vovelle, J.: Degenerate parabolic stochastic partial differential equations: Quasilinear case. Annals of Probability (2015)

14. Debussche, A., Vovelle, J.: Scalar conservation laws with stochastic forcing. J. Funct. Anal. 259(4), 1014-1042 (2010)

15. DiPerna, R.J.: Convergence of approximate solutions to conservation laws. Arch. Rational Mech. Anal. 82(1), 27-70 (1983)

16. Dotti, S., Vovelle, J.: Convergence of the finite volume method for scalar conservation laws with multiplicative noise: an approach by kinetic formulation. hal-01391073 (2016)

17. Droniou, J.: Intégration et espaces de sobolev à valeurs vectorielles (2001). http:// www-gm3.univ-mrs.fr/polys/

18. E, W., Khanin, K., Mazel, A., SinaI, Y.: Invariant measures for Burgers equation with stochastic forcing. Ann. of Math. (2) 151(3), 877-960 (2000)

19. Eymard, R., Gallouët, T., Herbin, R.: Finite volume methods. In: Handbook of numerical analysis, Vol. VII, Handb. Numer. Anal., VII, pages 713-1020. North-Holland, Amsterdam, 2000

20. Feng, J., Nualart, D.: Stochastic scalar conservation laws. J. Funct. Anal. 255(2), 313-373 (2008)

21. Gess, B., Perthame, B., Souganidis, P.E.: Semi-discretization for stochastic scalar conservation laws with multiple rough fluxes. SIAM Journal on Numerical Analysis 54(4), 2187-2209 (2016)

22. Gess, B., Souganidis, P.E.: Long-time behavior, invariant measures and regularizing effects for stochastic scalar conservation laws (Nov. 2014). arXiv:1411.3939 [math]

23. Gess, B., Souganidis, P.E.: Scalar conservation laws with multiple rough fluxes. Communications in Mathematical Sciences 13(6), 1569-1597 (2015)

24. GyÖNGY, I., KRYlOv, N.: Existence of strong solutions for Itô's stochastic equations via approximations. Probab. Theory Related Fields 105(2), 143-158 (1996)

25. Gyöngy, I., Rovira, C.: On $L^{p}$-solutions of semilinear stochastic partial differential equations. Stochastic Process. Appl. 90(1), 83-108 (2000)

26. Hofmanová, M.: Degenerate parabolic stochastic partial differential equations. Stochastic Process. Appl. 123(12), 4294-4336 (2013)

27. Hofmanová, M.: A Bhatnagar-Gross-Krook approximation to stochastic scalar conservation laws. Ann. Inst. Henri Poincaré Probab. Stat. 51(4), 1500-1528 (2015)

28. Hofmanová, M.: Scalar conservation laws with rough flux and stochastic forcing. Stochastic Partial Differential Equations. Analysis and Computations 4(3), 635-690 (2016)

29. Hofmanová, M., Seidler, J.: On weak solutions of stochastic differential equations. Stoch. Anal. Appl. 30(1), 100-121 (2012)

30. Jacod, J., Shiryaev, A.N.: Limit theorems for stochastic processes, volume 288 of Grundlehren der Mathematischen Wissenschaften [Fundamental Principles of Mathematical Sciences]. Springer-Verlag, Berlin, second edition, 2003

31. Karlsen, K.H., Storrøsten, E.B.: On stochastic conservation laws and Malliavin calculus. Journal of Functional Analysis 272(2), 421-497 (2017)

32. Kim, Y.: Asymptotic behavior of solutions to scalar conservation laws and optimal convergence orders to $N$-waves. J. Differential Equations 192(1), 202-224 (2003)

33. Koley, U., Majee, A.K., Vallet, G.: A finite difference scheme for conservation laws driven by Levy noise. arXiv:1604.07840 [math], (Apr. 2016)

34. KRÖKER, I., Rohde, C.: Finite volume schemes for hyperbolic balance laws with multiplicative noise. Appl. Numer. Math. 62(4), 441-456 (2012)

35. Lions, P.-L., Perthame, B., Souganidis, P.E.: Scalar conservation laws with rough (stochastic) fluxes. Stoch. Partial Differ. Equ. Anal. Comput. 1(4), 664-686 (2013) 
36. Lions, P.-L., Perthame, B., Souganidis, P.E.: Stochastic averaging lemmas for kinetic equations. In: Séminaire Laurent Schwartz-Équations aux dérivées partielles et applications. Année 2011-2012, Sémin. Équ. Dériv. Partielles, pages Exp. No. XXVI, 17. École Polytech., Palaiseau, 2013

37. Lions, P.-L., Perthame, B., Souganidis, P.E.: Scalar conservation laws with rough (stochastic) fluxes: the spatially dependent case. Stoch. Partial Differ. Equ. Anal. Comput. 2(4), 517-538 (2014)

38. Lions, P.-L., Perthame, B., Tadmor, E.: A kinetic formulation of multidimensional scalar conservation laws and related equations. J. Amer. Math. Soc. 7(1), 169-191 (1994)

39. Mohamed, K., Seaid, M., Zahri, M.: A finite volume method for scalar conservation laws with stochastic time-space dependent flux functions. J. Comput. Appl. Math. 237(1), 614-632 (2013)

40. OndREJÁt, M.: Stochastic nonlinear wave equations in local Sobolev spaces. Electron. J. Probab. 15(33), 1041-1091 (2010)

41. Perthame, B.: Kinetic formulation of conservation laws, volume 21 of Oxford Lecture Series in Mathematics and its Applications. Oxford University Press, Oxford, 2002

42. Revuz, D., Yor, M.: Continuous martingales and Brownian motion, volume 293 of Grundlehren der Mathematischen Wissenschaften [Fundamental Principles of Mathematical Sciences]. Springer-Verlag, Berlin, third edition, 1999

43. StorrøSTEN, E.B., KARLSEN, K.H.: Analysis of a splitting method for stochastic balance laws. arXiv:1601.02428 [math], (Jan. 2016)

44. Vallet, G., Wittbold, P.: On a stochastic first-order hyperbolic equation in a bounded domain. Infin. Dimens. Anal. Quantum Probab. Relat. Top. 12(4), 613-651 (2009)

45. Yosida, K.: Functional analysis, volume 123 of Grundlehren der Mathematischen Wissenschaften [Fundamental Principles of Mathematical Sciences]. Springer-Verlag, Berlin-New York, sixth edition, 1980

\author{
Sylvain DotTi \\ Aix-Marseille Université, CNRS, \\ Centrale Marseille, I2M, UMR 7373, \\ 13453, Marseille, France.
}

and

JULIEN VOVELLE

Univ Lyon, Université Claude Bernard Lyon 1, CNRS UMR 5208, Institut Camille Jordan, 43 blvd. du 11 novembre 1918, 69622, Villeurbanne Cedex, France. e-mail: vovelle@math.univ-lyon1.fr

(Received September 5, 2017 / Accepted April 25, 2018)

(C) Springer-Verlag GmbH Germany, part of Springer Nature (2018) 